\title{
Neutrosophic Soft Rough Graphs with Application
}

\author{
Muhammad Akram ${ }^{1, *(D)}$, Hafsa M. Malik ${ }^{1}$, Sundas Shahzadi ${ }^{1}$ and Florentin Smarandache 2 (iD \\ 1 Department of Mathematics, University of the Punjab, New Campus, Lahore 54590, Pakistan; \\ hafsa.masood.malik@gmail.com (H.M.M.); sundas1011@gmail.com (S.S.) \\ 2 Mathematics \& Science Department, University of New Mexico, 705 Gurley Ave., Gallup, NM 87301, USA; \\ fsmarandache@gmail.com \\ * Correspondence: m.akram@pucit.edu.pk; Tel.: +92-42-99231241
}

Received: 27 January 2018; Accepted: 23 February 2018; Published: 26 February 2018

\begin{abstract}
Neutrosophic sets (NSs) handle uncertain information while fuzzy sets (FSs) and intuitionistic fuzzy sets (IFs) fail to handle indeterminate information. Soft set theory, neutrosophic set theory, and rough set theory are different mathematical models for handling uncertainties and they are mutually related. The neutrosophic soft rough set (NSRS) model is a hybrid model by combining neutrosophic soft sets with rough sets. We apply neutrosophic soft rough sets to graphs. In this research paper, we introduce the idea of neutrosophic soft rough graphs (NSRGs) and describe different methods of their construction. We consider the application of NSRG in decision-making problems. In particular, we develop efficient algorithms to solve decision-making problems.
\end{abstract}

Keywords: neutrosophic soft rough sets; neutrosophic soft rough graphs; decision-making; algorithm

\section{Introduction}

Smarandache [1] initiated the concept of neutrosophic set (NS). Smarandache's NS is characterized by three parts: truth, indeterminacy, and falsity. Truth, indeterminacy and falsity membership values behave independently and deal with problems having uncertain, indeterminant and imprecise data. Wang et al. [2] gave a new concept of single valued neutrosophic sets (SVNSs) and defined the set theoretic operators on an instance of NS called SVNS. Peng et al. [3] discussed the operations of simplified neutrosophic numbers and introduced an outranking idea of simplified neutrosophic numbers.

Molodtsov [4] introduced the notion of soft set (SS) as a novel mathematical approach for handling uncertainties. Molodtsov's SSs gave us a new technique for dealing with uncertainty from the viewpoint of parameters. Maji et al. [5-7] introduced neutrosophic soft sets (NSSs), intuitionistic fuzzy soft sets and fuzzy soft sets (FSSs). In [8], Sahin and Kucuk presented NSS in the form of neutrosophic relations.

Theory of rough set (RS) was proposed by Pawlak [9] in 1982. Rough set theory is used to study the intelligence systems containing incomplete, uncertain or inexact information. The lower and upper approximation operators of RSs are used for managing hidden information in a system. Feng et al. [10] took a significant step to introduce parametrization tools in RSs. Meng et al. [11] provide further discussion of the combination of SSs, RSs and FSs. The existing results of RSs and other extended RSs such as rough fuzzy sets, generalized rough fuzzy sets, soft fuzzy rough sets and intuitionistic fuzzy rough sets based decision-making models have their advantages and limitations [12,13]. In a different way, rough set approximations have been constructed into the intuitionistic fuzzy environment and are known as intuitionistic fuzzy rough sets and rough intuitionistic fuzzy sets $[14,15]$. Zhang et al. [16,17] presented the notions of soft rough sets, soft rough intuitionistic fuzzy sets, intuitionistic fuzzy soft rough sets, its application in decision-making, and also introduced generalized intuitionistic fuzzy soft rough sets. Broumi et al. $[18,19]$ developed a hybrid structure by combining 
RSs and NSs, called RNSs, they also presented interval valued neutrosophic soft rough sets by combining interval valued neutrosophic soft sets and RSs. Yang et al. [20] proposed single valued neutrosophic rough sets (SVNRSs) by combining SVNSs and RSs and defined SVNRSs on two universes and established an algorithm for a decision-making problem based on SVNRSs on two universes. Akram and Nawaz [21] have introduced the concept of soft graphs and some operation on soft graphs. Certain concepts of fuzzy soft graphs and intuitionistic fuzzy soft graphs are discussed in [22-24]. Akram and Shahzadi [25] have introduced neutrosophic soft graphs. Zafar and Akram [26] introduced a rough fuzzy digraph and several basic notions concerning rough fuzzy digraphs. In this research paper, a neutrosophic soft rough set is a generalization of a neutrosophic rough set, and we introduce the idea of neutrosophic soft rough graphs (NSRGs) that are made by combining NSRSs with graphs and describe different methods of their construction. We consider the application of NSRG in decision-making problems and resolve the problem. In particular, we develop efficient algorithms to solve decision-making problems.

For other notations, terminologies and applications not mentioned in the paper, the readers are referred to [27-35].

\section{Neutrosophic Soft Rough Information}

In this section, we will introduce the notions of neutrosophic soft rough relation (NSRR), and NSRGs.

Definition 1. Let $Y$ be an initial universal set, $\mathbb{P}$ a universal set of parameters and $\mathbb{M} \subseteq \mathbb{P}$. For an arbitrary neutrosophic soft relation $Q$ over $Y \times \mathbb{M},(Y, \mathbb{M}, Q)$ is called neutrosophic soft approximation space (NSAS).

For any NS $A \in \mathcal{N}(\mathbb{M})$, we define the upper neutrosophic soft rough approximation (UNSRA) and the lower neutrosophic soft rough approximation (LNSRA) operators of $A$ with respect to $(Y, \mathbb{M}, Q)$ denoted by $\bar{Q}(A)$ and $Q(A)$, respectively as follows:

$$
\begin{aligned}
& \bar{Q}(A)=\left\{\left(u, T_{\bar{Q}(A)}(u), I_{\bar{Q}(A)}(u), F_{\bar{Q}(A)}(u)\right) \mid u \in Y\right\}, \\
& \underline{Q}(A)=\left\{\left(u, T_{\underline{Q}(A)}(u), I_{\underline{Q}(A)}(u), F_{\underline{Q}(A)}(u)\right) \mid u \in Y\right\},
\end{aligned}
$$

where

$$
\begin{array}{ll}
T_{\bar{Q}(A)}(u)= & \bigvee_{e \in \mathbb{M}}\left(T_{Q(A)}(u, e) \wedge T_{A}(e)\right), \quad I_{\bar{Q}(A)}(u)=\bigwedge_{e \in \mathbb{M}}\left(I_{Q(A)}(u, e) \vee I_{A}(e)\right), \\
F_{\bar{Q}(A)}(u)= & \bigwedge_{e \in \mathbb{M}}\left(F_{Q(A)}(u, e) \vee F_{A}(e)\right) ; \quad T_{\underline{Q}(A)}(u)=\bigwedge_{e \in \mathbb{M}}\left(F_{Q(A)}(u, e) \vee T_{A}(e)\right), \\
I_{Q}(A) & (u)=\bigvee_{e \in \mathbb{M}}\left(\left(1-I_{Q(A)}(u, e)\right) \wedge I_{A}(e)\right), F_{\underline{Q}(A)}(u)=\bigvee_{e \in \mathbb{M}}\left(T_{Q(A)}(u, e) \wedge F_{A}(e)\right) .
\end{array}
$$

The pair $(Q(A), \bar{Q}(A))$ is called NSRS of $A$ w.r.t $(Y, \mathbb{M}, Q), \underline{Q}$ and $\bar{Q}$ are referred to as the LNSRA and the UNSRA operators, respectively.

Example 1. Suppose that $Y=\left\{w_{1}, w_{2}, w_{3}, w_{4}\right\}$ is the set of careers under consideration, and Mr. $X$ wants to select the best suitable career. $\mathbb{M}=\left\{e_{1}, e_{2}, e_{3}\right\}$ is a set of decision parameters. Mr. X describes the "most suitable career" by defining a neutrosophic soft set $(Q, \mathbb{M})$ on $Y$ that is a neutrosophic relation from $Y$ to $\mathbb{M}$ as shown in Table 1.

Table 1. Neutrosophic soft relation $Q$.

\begin{tabular}{ccccc}
\hline$Q$ & $w_{1}$ & $w_{\mathbf{2}}$ & $w_{3}$ & $w_{4}$ \\
\hline$e_{1}$ & $(0.3,0.4,0.5)$ & $(0.4,0.2,0.3)$ & $(0.1,0.5,0.4)$ & $(0.2,0.3,0.4)$ \\
$e_{2}$ & $(0.1,0.5,0.4)$ & $(0.3,0.4,0.6)$ & $(0.4,0.4,0.3)$ & $(0.5,0.3,0.8)$ \\
$e_{3}$ & $(0.3,0.4,0.4)$ & $(0.4,0.6,0.7)$ & $(0.3,0.5,0.4)$ & $(0.5,0.4,0.6)$ \\
\hline
\end{tabular}


Now, Mr. X gives the most favorable decision object $A$, which is an NS on $\mathbb{M}$ defined as follows: $A=$ $\left\{\left(e_{1}, 0.5,0.2,0.4\right),\left(e_{2}, 0.2,0.3,0.1\right),\left(e_{3}, 0.2,0.4,0.6\right)\right\}$. By Definition 1 , we have

$$
\begin{array}{lll}
T_{\bar{Q}(A)}\left(w_{1}\right)=0.3, & I_{\bar{Q}(A)}\left(w_{1}\right)=0.4, & F_{\bar{Q}(A)}\left(w_{1}\right)=0.4, \\
T_{\bar{Q}(A)}\left(w_{2}\right)=0.4, & I_{\bar{Q}(A)}\left(w_{2}\right)=0.2, & F_{\bar{Q}(A)}\left(w_{2}\right)=0.4, \\
T_{\bar{Q}(A)}\left(w_{3}\right)=0.2, & I_{\bar{Q}(A)}\left(w_{3}\right)=0.4, & F_{\bar{Q}(A)}\left(w_{3}\right)=0.3, \\
T_{\bar{Q}(A)}\left(w_{4}\right)=0.2, & I_{\bar{Q}(A)}\left(w_{4}\right)=0.3, & F_{\bar{Q}(A)}\left(w_{4}\right)=0.4 .
\end{array}
$$

Similarly,

$$
\begin{array}{ccc}
T_{\underline{Q}(A)}\left(w_{1}\right)=0.4, & I_{\underline{Q}(A)}\left(w_{1}\right)=0.4, & F_{\underline{Q}(A)}\left(w_{1}\right)=0.3, \\
T_{\underline{Q}(A)}\left(w_{2}\right)=0.5, & I_{\underline{Q}(A)}\left(w_{2}\right)=0.4, & F_{\underline{Q}(A)}\left(w_{2}\right)=0.4, \\
T_{\underline{Q}(A)}\left(w_{3}\right)=0.4, & I_{\underline{Q}(A)}\left(w_{3}\right)=0.4, & F_{\underline{Q}(A)}\left(w_{3}\right)=0.3, \\
T_{\underline{Q}(A)}\left(w_{4}\right)=0.5, & I_{\underline{Q}(A)}\left(w_{4}\right)=0.4, & F_{\underline{Q}(A)}\left(w_{4}\right)=0.5 .
\end{array}
$$

Thus, we obtain

$$
\begin{aligned}
& \bar{Q}(A)=\left\{\left(w_{1}, 0.3,0.4,0.4\right),\left(w_{2}, 0.4,0.2,0.4\right),\left(w_{3}, 0.2,0.4,0.3\right),\left(w_{4}, 0.2,0.3,0.4\right)\right\}, \\
& \underline{Q}(A)=\left\{\left(w_{1}, 0.4,0.4,0.3\right),\left(w_{2}, 0.5,0.4,0.4\right),\left(w_{3}, 0.4,0.4,0.3\right),\left(w_{4}, 0.5,0.4,0.5\right)\right\} .
\end{aligned}
$$

Hence, $(\underline{Q}(A), \bar{Q}(A))$ is an NSRS of $A$.

The conventional neutrosophic soft set is a mapping from a parameter to the neutrosophic subset of the universe and letting $(Q, \mathbb{M})$ be neutrosophic soft set. Now, we present the constructive definition of neutrosophic soft rough relation by using a neutrosphic soft relation $S$ from $\mathbb{M} \times \mathbb{M}=\mathbb{M}$ to $\mathcal{N}(Y \times Y=Y)$, where $Y$ is a universal set and $\mathbb{M}$ be a set of parameters.

Definition 2. A neutrosophic soft rough relation $(\underline{S}(B), \bar{S}(B))$ on $Y$ is an $N S R S, S: \mathbb{M} \rightarrow \mathcal{N}(\bar{Y})$ is a neutrosophic soft relation on $Y$ defined by

$$
\begin{aligned}
S\left(e_{i} e_{j}\right) & =\left\{u_{i} u_{j} \mid \exists u_{i} \in Q\left(e_{i}\right), u_{j} \in Q\left(e_{j}\right)\right\}, u_{i} u_{j} \in \dot{Y}, \text { such that } \\
T_{S}\left(u_{i} u_{j}, e_{i} e_{j}\right) & \leqslant \min \left\{T_{Q}\left(u_{i}, e_{i}\right), T_{Q}\left(u_{j}, e_{j}\right)\right\} \\
I_{S}\left(u_{i} u_{j}, e_{i} e_{j}\right) & \leqslant \max \left\{I_{Q}\left(u_{i}, e_{i}\right), I_{Q}\left(u_{j}, e_{j}\right)\right\} \\
F_{S}\left(u_{i} u_{j}, e_{i} e_{j}\right) & \leqslant \max \left\{F_{Q}\left(u_{i}, e_{i}\right), F_{Q}\left(u_{j}, e_{j}\right)\right\} . \\
\text { For any } B \in \mathcal{N}(\mathbb{M}), B & =\left\{\left(e_{i} e_{j}, T_{B}\left(e_{i} e_{j}\right), I_{B}\left(e_{i} e_{j}\right), F_{B}\left(e_{i} e_{j}\right)\right) u_{i} u_{j} \in \mathbb{M}\right\}, \\
T_{B}\left(e_{i} e_{j}\right) & \leqslant \min \left\{T_{A}\left(e_{i}\right), T_{A}\left(e_{j}\right)\right\}, \\
I_{B}\left(e_{i} e_{j}\right) & \leqslant \max \left\{I_{A}\left(e_{i}\right), I_{A}\left(e_{j}\right)\right\}, \\
F_{B}\left(e_{i} e_{j}\right) & \leqslant \max \left\{F_{A}\left(e_{i}\right), F_{A}\left(e_{j}\right)\right\} .
\end{aligned}
$$

The UNSA and the LNSA of B w.r.t $(\dot{Y}, \mathbb{M}, S)$ are defined as follows:

$$
\begin{aligned}
& \bar{S}(B)=\left\{\left(u_{i} u_{j}, T_{\bar{S}(B)}\left(u_{i} u_{j}\right), I_{\bar{S}(B)}\left(u_{i} u_{j}\right), F_{\bar{S}(B)}\left(u_{i} u_{j}\right)\right) \mid u_{i} u_{j} \in \tilde{Y}\right\}, \\
& \underline{S}(B)=\left\{\left(u_{i} u_{j}, T_{\underline{S}(B)}\left(u_{i} u_{j}\right), I_{\underline{S}(B)}\left(u_{i} u_{j}\right), F_{\underline{S}(B)}\left(u_{i} u_{j}\right)\right) \mid u_{i} u_{j} \in \tilde{Y}\right\},
\end{aligned}
$$


where

$$
\begin{aligned}
T_{\bar{S}(B)}\left(u_{i} u_{j}\right) & =\bigvee_{e_{i} e_{j} \in \mathbb{\mathbb { M }}}\left(T_{S}\left(u_{i} u_{j}, e_{i} e_{j}\right) \wedge T_{B}\left(e_{i} e_{j}\right)\right), \\
I_{\bar{S}(B)}\left(u_{i} u_{j}\right) & =\bigwedge_{e_{i} e_{j} \in \mathbb{\mathbb { M }}}\left(I_{S}\left(u_{i} u_{j}, e_{i} e_{j}\right) \vee I_{B}\left(e_{i} e_{j}\right)\right), \\
F_{\bar{S}(B)}\left(u_{i} u_{j}\right) & =\bigwedge_{e_{i} e_{j} \in \tilde{\mathbb{M}}}\left(F_{S}\left(u_{i} u_{j}, e_{i} e_{j}\right) \vee F_{B}\left(e_{i} e_{j}\right)\right) ; \\
T_{\underline{S}(B)}\left(u_{i} u_{j}\right) & =\bigwedge_{e_{i} e_{j} \in \mathbb{\mathbb { M }}}\left(F_{S}\left(u_{i} u_{j}, e_{i} e_{j}\right) \vee T_{B}\left(e_{i} e_{j}\right)\right), \\
I_{\underline{S}(B)}\left(u_{i} u_{j}\right) & =\bigvee_{e_{i} e_{j} \in \mathbb{\mathbb { M }}}\left(\left(1-I_{S}\left(u_{i} u_{j}, e_{i} e_{j}\right)\right) \wedge I_{B}\left(e_{i} e_{j}\right)\right), \\
F_{\underline{S}(B)}\left(u_{i} u_{j}\right) & =\bigvee_{e_{i} e_{j} \in \tilde{\mathbb{M}}}\left(T_{S}\left(u_{i} u_{j}, e_{i} e_{j}\right) \wedge F_{B}\left(e_{i} e_{j}\right)\right) .
\end{aligned}
$$

The pair $(\underline{S}(B), \bar{S}(B))$ is called NSRR and $\underline{S}, \bar{S}: \mathcal{N}(\mathbb{M}) \rightarrow \mathcal{N}(\bar{Y})$ are called the LNSRA and the UNSRA operators, respectively.

Remark 1. Consider an NS B on $\mathbb{M}$ and an NS A on $\mathbb{M}$, according to the definition of NSRR, we get

$$
\begin{aligned}
T_{\bar{S}(B)}\left(u_{i} u_{j}\right) & \leqslant \min \left\{T_{\bar{S}(A)}\left(u_{i}\right), T_{\bar{S}(A)}\left(u_{j}\right)\right\} \\
I_{\bar{S}(B)}\left(u_{i} u_{j}\right) & \leqslant \max \left\{I_{\bar{S}(A)}\left(u_{i}\right), I_{\bar{S}(A)}\left(u_{j}\right)\right\} \\
F_{\bar{S}(B)}\left(u_{i} u_{j}\right) & \leqslant \max \left\{F_{\bar{S}(A)}\left(u_{i}\right) \cdot F_{\bar{S}(A)}\left(u_{j}\right)\right\} .
\end{aligned}
$$

Similarly, for LNSRA operator $\underline{S}(B)$,

$$
\begin{aligned}
T_{\underline{S}(B)}\left(u_{i} u_{j}\right) & \leqslant \min \left\{T_{\underline{S}(A)}\left(u_{i}\right), T_{\underline{S}(A)}\left(u_{j}\right)\right\} \\
I_{\underline{S}(B)}\left(u_{i} u_{j}\right) & \leqslant \max \left\{I_{\underline{S}(A)}\left(u_{i}\right), I_{\underline{S}(A)}\left(u_{j}\right)\right\} \\
F_{\underline{S}(B)}\left(u_{i} u_{j}\right) & \leqslant \max \left\{F_{\underline{S}(A)}\left(u_{i}\right) \cdot F_{\underline{S}(A)}\left(u_{j}\right)\right\} .
\end{aligned}
$$

Example 2. Let $Y=\left\{u_{1}, u_{2}, u_{3}\right\}$ be a universal set and $\mathbb{M}=\left\{e_{1}, e_{2}, e_{3}\right\}$ a set of parameters. A neutrosophic soft set $(Q, \mathbb{M})$ on $Y$ can be defined in tabular form in Table 2 as follows:

Table 2. Neutrosophic soft set $(Q, \mathbb{M})$.

\begin{tabular}{cccc}
\hline $\boldsymbol{Q}$ & $\boldsymbol{u}_{\mathbf{1}}$ & $\boldsymbol{u}_{\mathbf{2}}$ & $\boldsymbol{u}_{\mathbf{3}}$ \\
\hline$e_{1}$ & $(0.4,0.5,0.6)$ & $(0.7,0.3,0.2)$ & $(0.6,0.3,0.4)$ \\
$e_{2}$ & $(0.5,0.3,0.6)$ & $(0.3,0.4,0.3)$ & $(0.7,0.2,0.3)$ \\
$e_{3}$ & $(0.7,0.2,0.3)$ & $(0.6,0.5,0.4)$ & $(0.7,0.2,0.4)$ \\
\hline
\end{tabular}

Let $E=\left\{u_{1} u_{2}, u_{2} u_{3}, u_{2} u_{2}, u_{3} u_{2}\right\} \subseteq \dot{Y}$ and $L=\left\{e_{1} e_{3}, e_{2} e_{1}, e_{3} e_{2}\right\} \subseteq \mathbb{M}$. Then, a soft relation $S$ on $E$ (from $L$ to $E$ ) can be defined in Table 3 as follows: 
Table 3. Neutrosophic soft relation $S$.

\begin{tabular}{ccccc}
\hline $\boldsymbol{S}$ & $\boldsymbol{u}_{\mathbf{1}} \boldsymbol{u}_{\mathbf{2}}$ & $\boldsymbol{u}_{\mathbf{2}} \boldsymbol{u}_{\mathbf{3}}$ & $\boldsymbol{u}_{\mathbf{2}} \boldsymbol{u}_{\mathbf{2}}$ & $\boldsymbol{u}_{3} \boldsymbol{u}_{\mathbf{2}}$ \\
\hline$e_{1} e_{3}$ & $(0.4,0.4,0.5)$ & $(0.6,0.3,0.4)$ & $(0.5,0.4,0.2)$ & $(0.5,0.4,0.3)$ \\
$e_{2} e_{1}$ & $(0.3,0.3,0.4)$ & $(0.3,0.2,0.3)$ & $(0.2,0.3,0.3)$ & $(0.7,0.2,0.2)$ \\
$e_{3} e_{2}$ & $(0.3,0.3,0.2)$ & $(0.5,0.3,0.2)$ & $(0.2,0.4,0.4)$ & $(0.3,0.4,0.4)$ \\
\hline
\end{tabular}

Let $A=\left\{\left(e_{1}, 0.2,0.4,0.6\right),\left(e_{2}, 0.4,0.5,0.2\right),\left(e_{3}, 0.1,0.2,0.4\right)\right\}$ be an $N S$ on $\mathbb{M}$, then $\bar{S}(A)=\left\{\left(u_{1}, 0.4,0.2,0.4\right),\left(u_{2}, 0.3,0.4,0.3\right),\left(u_{3}, 0.4,0.2,0.3\right)\right\}$, $\underline{S}(A)=\left\{\left(u_{1}, 0.3,0.5,0.4\right),\left(u_{2}, 0.2,0.5,0.6\right),\left(u_{3}, 0.4,0.5,0.6\right)\right\}$. Let $B=\left\{\left(e_{1} e_{3}, 0.1,0.3,0.5\right),\left(e_{2} e_{1}, 0.2,0.4,0.3\right),\left(e_{3} e_{2}, 0.1,0.2,0.3\right)\right\}$ be an NS on $L$, then $\bar{S}(B)=\left\{\left(u_{1} u_{2}, 0.2,0.3,0.3\right),\left(u_{2} u_{3}, 0.2,0.3,0.3\right),\left(u_{2} u_{2}, 0.2,0.4,0.3\right),\left(u_{3} u_{2}, 0.2,0.4,0.3\right)\right\}$, $\underline{S}(B)=\left\{\left(u_{1} u_{2}, 0.2,0.4,0.4\right),\left(u_{2} u_{3}, 0.2,0.4,0.5\right),\left(u_{2} u_{2}, 0.3,0.4,0.5\right),\left(u_{3} u_{2}, 0.2,0.4,0.5\right)\right\}$. Hence, $S(B)=(\underline{S}(B), \bar{S}(B))$ is NSRR.

Definition 3. A neutrosophic soft rough graph (NSRG) on a non-empty $V$ is an 4-ordered tuple $(V, \mathbb{M}, Q(A), S(B))$ such that

(i) $\mathbb{M}$ is a set of parameters,

(ii) $Q$ is an arbitrary neutrosophic soft relation over $V \times \mathbb{M}$,

(iii) $S$ is an arbitrary neutrosophic soft relation over $V \times \mathbb{M}$,

(vi) $Q(A)=(Q A, \bar{Q} A)$ is an NSRS of $A$,

(v) $S(B)=(\underline{S B}, \bar{S} B)$ is an NSRR on $\bar{V} \subset V \times V$,

(iv) $G=(Q(A), S(B))$ is a neutrosophic soft rough graph, where $\underline{G}=(\underline{Q} A, \underline{S} B)$ and $\bar{G}=(\bar{Q} A, \bar{S} B)$ are lower neutrosophic approximate graph (LNAG) and upper neutrosophic approximate graph (UNAG), respectively of neutrosophic soft rough graph (NSRG) $G=(Q(A), S(B))$.

Example 3. Let $V=\left\{v_{1}, v_{2}, v_{3}, v_{4}, v_{5}, v_{6}\right\}$ be a vertex set and $\mathbb{M}=\left\{e_{1}, e_{2}, e_{3}\right\}$ a set of parameters. A neutrosophic soft relation over $V \times \mathbb{M}$ can be defined in tabular form in Table 4 as follows:

Table 4. Neutrosophic soft relation $Q$.

\begin{tabular}{ccccccc}
\hline $\boldsymbol{Q}$ & $\boldsymbol{v}_{\mathbf{1}}$ & $\boldsymbol{v}_{\mathbf{2}}$ & $\boldsymbol{v}_{\mathbf{3}}$ & $\boldsymbol{v}_{\mathbf{4}}$ & $\boldsymbol{v}_{5}$ & $\boldsymbol{v}_{\mathbf{6}}$ \\
\hline$e_{1}$ & $(0.4,0.5,0.6)$ & $(0.7,0.3,0.5)$ & $(0.6,0.2,0.3)$ & $(0.4,0.4,0.2)$ & $(0.5,0.5,0.6)$ & $(0.4,0.5,0.6)$ \\
$e_{2}$ & $(0.5,0.4,0.2)$ & $(0.6,0.4,0.5)$ & $(0.7,0.3,0.4)$ & $(0.5,0.3,0.2)$ & $(0.4,0.5,0.4)$ & $(0.6,0.5,0.4)$ \\
$e_{3}$ & $(0.5,0.4,0.1)$ & $(0.6,0.3,0.2)$ & $(0.5,0.4,0.3)$ & $(0.6,0.2,0.3)$ & $(0.5,0.4,0.4)$ & $(0.7,0.3,0.5)$ \\
\hline
\end{tabular}

Let $A=\left\{\left(e_{1}, 0.5,0.4,0.6\right),\left(e_{2}, 0.7,0.4,0.5\right),\left(e_{3}, 0.6,0.2,0.5\right)\right\}$ be an $N S$ on $\mathbb{M}$, then

$$
\begin{aligned}
\bar{S}(A)= & \left\{\left(v_{1}, 0.5,0.4,0.5\right),\left(v_{2}, 0.6,0.3,0.5\right),\left(v_{3}, 0.7,0.4,0.5\right),\left(v_{4}, 0.6,0.2,0.5\right),\left(v_{5}, 0.5,\right.\right. \\
& \left.0.4,0.5),\left(v_{6}, 0.6,0.3,0.5\right)\right\}, \\
\underline{S}(A)= & \left\{\left(v_{1}, 0.6,0.4,0.5\right),\left(v_{2}, 0.5,0.4,0.6\right),\left(v_{3}, 0.5,0.4,0.6\right),\left(v_{4}, 0.5,0.4,0.5\right),\left(v_{5}, 0.6,\right.\right. \\
& \left.0.4,0.5),\left(v_{6}, 0.6,0.4,0.5\right)\right\} .
\end{aligned}
$$

Let $E=\left\{v_{1} v_{1}, v_{1} v_{2}, v_{2} v_{1}, v_{2} v_{3}, v_{4} v_{5}, v_{3} v_{4}, v_{5} v_{2}, v_{5} v_{6}\right\} \subseteq \dot{V}$ and $L=\left\{e_{1} e_{3}, e_{2} e_{1}, e_{3} e_{2}\right\} \subseteq \mathbb{M}$. Then, a neutrosophic soft relation $S$ on $E$ (from $L$ to $E$ ) can be defined in Tables 5 and 6 as follows:

Table 5. Neutrosophic soft relation $S$.

\begin{tabular}{ccccc}
\hline$S$ & $v_{1} v_{1}$ & $v_{1} v_{2}$ & $v_{2} v_{1}$ & $v_{2} v_{3}$ \\
\hline$e_{1} e_{2}$ & $(0.4,0.4,0.2)$ & $(0.4,0.4,0.5)$ & $(0.4,0.4,0.5)$ & $(0.6,0.3,0.4)$ \\
$e_{2} e_{3}$ & $(0.5,0.4,0.1)$ & $(0.4,0.3,0.2)$ & $(0.4,0.3,0.2)$ & $(0.5,0.3,0.2)$ \\
$e_{1} e_{3}$ & $(0.4,0.4,0.1)$ & $(0.4,0.2,0.2)$ & $(0.4,0.2,0.2)$ & $(0.5,0.3,0.3)$ \\
\hline
\end{tabular}


Table 6. Neutrosophic soft relation $S$.

\begin{tabular}{ccccc}
\hline$S$ & $v_{3} v_{4}$ & $v_{4} v_{5}$ & $v_{5} v_{2}$ & $v_{5} v_{6}$ \\
\hline$e_{1} e_{2}$ & $(0.4,0.2,0.2)$ & $(0.4,0.4,0.2)$ & $(0.4,0.3,0.4)$ & $(0.3,0.2,0.3)$ \\
$e_{2} e_{3}$ & $(0.6,0.2,0.4)$ & $(0.3,0.2,0.1)$ & $(0.4,0.3,0.2)$ & $(0.4,0.3,0.4)$ \\
$e_{1} e_{3}$ & $(0.4,0.2,0.3)$ & $(0.4,0.3,0.1)$ & $(0.5,0.3,0.2)$ & $(0.5,0.3,0.5)$ \\
\hline
\end{tabular}

Let $B=\left\{\left(e_{1} e_{2}, 0.4,0.4,0.5 ;\right),\left(e_{2} e_{3}, 0.5,0.4,0.5\right),\left(e_{1} e_{3}, 0.5,0.2,0.5\right)\right\}$ be an NS on $\mathrm{L}$, then

$\bar{S} B=\left\{\left(v_{1} v_{1}, 0.5,0.4,0.5\right),\left(v_{1} v_{2}, 0.4,0.2,0.5\right),\left(v_{2} v_{1}, 0.4,0.2,0.5\right),\left(v_{2} v_{3}, 0.5,0.3,0.5\right)\right.$, $\left.\left(v_{3} v_{4}, 0.5,0.2,0.5\right),\left(v_{4} v_{5}, 0.4,0.3,0.5\right),\left(v_{5} v_{2}, 0.5,0.3,0.5\right),\left(v_{5} v_{6}, 0.5,0.3,0.5\right)\right\}$ ，

$\underline{S} B=\left\{\left(v_{1} v_{1}, 0.4,0.4,0.5\right)\left(v_{1} v_{2}, 0.5,0.4,0.4\right),\left(v_{2} v_{1}, 0.5,0.4,0.4\right),\left(v_{2} v_{3}, 0.4,0.4,0.5\right)\right.$, $\left.\left(v_{3} v_{4}, 0.4,0.4,0.5\right),\left(v_{4} v_{5}, 0.4,0.4,0.4\right),\left(v_{5} v_{2}, 0.4,0.4,0.5\right),\left(v_{5} v_{6}, 0.4,0.4,0.5\right)\right\}$.

Hence, $S(B)=(\underline{S} B, \bar{S} B)$ is NSRR on $\bar{V}$.

Thus, $\underline{G}=(\underline{Q} A, \underline{S} B)$ and $\bar{G}=(\bar{Q} A, \bar{S} B)$ are LNAG and $U N A G$, respectively, are shown in Figure 1 .

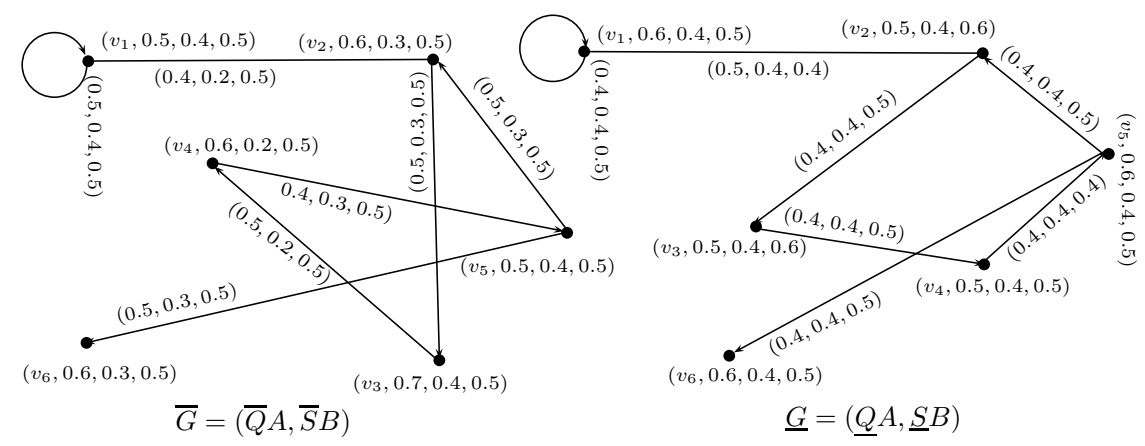

Figure 1. Neutrosophic soft rough graph $G=(\underline{G}, \bar{G})$

Hence, $G=(\underline{G}, \bar{G})$ is NSRG.

Definition 4. Let $G=(V, \mathbb{M}, Q, S)$ be a neutrosophic soft rough graph on a non-empty set $V$. The order of $G$ can be denoted by $\mathbf{O}(G)$, defined by

$$
\begin{aligned}
& \mathbf{O}(G)=\mathbf{O}(\bar{G})+\mathbf{O}(\underline{G}), \text { where } \\
& \mathbf{O}(\bar{G})=\sum_{v \in V} \bar{Q} A(v), \mathbf{O}(\underline{G})=\sum_{v \in V} \underline{Q} A(v) .
\end{aligned}
$$

The size of neutrosophic soft rough graph $G$, denoted by $\mathbf{S}(G)$, defined by

$$
\begin{aligned}
& \mathbf{S}(G)=(\mathbf{S} \bar{G}+\mathbf{S} \underline{G}), \text { where } \\
& \mathbf{S}(\bar{G})=\sum_{u v \in E} \bar{S} B(u v), \mathbf{S}(\underline{G})=\sum_{u v \in E} \underline{S} B(u v) .
\end{aligned}
$$

Example 4. Let $G$ be a neutrosophic soft rough graph as shown in Figure 1. Then,

$$
\begin{aligned}
& \mathbf{O}(\bar{G})=(3.5,2.0,3.0), \mathbf{O}(\underline{G})=(3.3,2.4,3.2), \\
& \mathbf{O}(G)=\mathbf{O}(\bar{G})+\mathbf{O}(\underline{G})=(6.8,4.4,6.2), \text { and } \\
& \mathbf{S}(\bar{G})=(3.2,1.8,3.0) \mathbf{S}(\underline{G})=(2.5,2.4,2.8) \\
& \mathbf{S}(G)=\mathbf{S}(\bar{G})+\mathbf{S}(\underline{G})=(5.7,4.2,5.8) .
\end{aligned}
$$


Definition 5. Let $G_{1}=\left(\underline{G}_{1}, \bar{G}_{1}\right)$ and $G_{2}=\left(\underline{G}_{2}, \bar{G}_{2}\right)$ be two neutrosophic soft rough graphs on $V$. The union of $G_{1}$ and $G_{2}$ is a neutrosophic soft rough graph $G=G_{1} \cup G_{2}=\left(\underline{G}_{1} \cup \underline{G}_{2}, \bar{G}_{1} \cup \bar{G}_{2}\right)$, where $\underline{G}_{1} \cup \underline{G}_{2}=$ $\left(\underline{Q} A_{1} \cup \underline{Q} A_{2}, \underline{S} B_{1} \cup \underline{S} B_{2}\right)$ and $\bar{G}_{1} \cup \bar{G}_{2}=\left(\bar{Q} A_{1} \cup \bar{Q} A_{2}, \bar{S} B_{1} \cup \bar{S} B_{2}\right)$ are neutrosophic graphs, such that

(i) $\forall v \in Q A_{1}$ but $v \notin Q A_{2}$.

$$
\begin{gathered}
T_{\overline{\mathrm{Q}} A_{1} \cup \overline{\mathrm{Q}} A_{2}}(v)=T_{\overline{\mathrm{Q}} A_{1}}(v), T_{\underline{Q} A_{1} \cup \underline{Q} A_{2}}(v)=T_{\underline{Q} A_{1}}(v), \\
I_{\overline{\mathrm{Q}} A_{1} \cup \overline{\mathrm{Q}} A_{2}}(v)=I_{\overline{\mathrm{Q}} A_{1}}(v), I_{\underline{Q} A_{1} \cup \underline{Q} A_{2}}(v)=I_{\underline{Q} A_{1}}(v), \\
F_{\overline{\mathrm{Q}} A_{1} \cup \overline{\mathrm{Q}} A_{2}}(v)=F_{\overline{\mathrm{Q}} A_{1}}(v), F_{\underline{Q} A_{1} \cup \underline{Q} A_{2}}(v)=F_{\underline{Q} A_{1}}(v) .
\end{gathered}
$$

(ii) $\forall v \notin Q A_{1}$ but $v \in Q A_{2}$.

$$
\begin{gathered}
T_{\overline{\mathrm{Q}} A_{1} \cup \overline{\mathrm{Q}} A_{2}}(v)=T_{\overline{\mathrm{Q}} A_{2}}(v), T_{\underline{Q} A_{1} \cup \underline{Q} A_{2}}(v)=T_{\underline{Q} A_{2}}(v), \\
I_{\overline{\mathrm{Q}} A_{1} \cup \overline{\mathrm{Q}} A_{2}}(v)=I_{\overline{\mathrm{Q}} A_{2}}(v), I_{\underline{Q} A_{1} \cup \underline{Q} A_{2}}(v)=I_{\underline{Q} A_{2}}(v), \\
F_{\bar{Q} A_{1} \cup \overline{\mathrm{Q}} A_{2}}(v)=F_{\bar{Q} A_{2}}(v), F_{\underline{Q} A_{1} \cup \underline{Q} A_{2}}(v)=F_{\underline{Q} A_{2}}(v) .
\end{gathered}
$$

(iii) $\forall v \in Q A_{1} \cap \underline{Q} A_{2}$

$$
\begin{gathered}
T_{\overline{\mathrm{Q}} A_{1} \cup \bar{Q} A_{2}}(v)=\max \left\{T_{\overline{\mathrm{Q}} A_{1}}(v), T_{\overline{\mathrm{Q}} A_{2}}(v)\right\}, T_{\underline{Q} A_{1} \cup \underline{Q} A_{2}}(v)=\max \left\{T_{\underline{Q} A_{1}}(v), T_{\underline{Q} A_{2}}(v)\right\}, \\
I_{\overline{\mathrm{Q}} A_{1} \cup \bar{Q} A_{2}}(v)=\min \left\{I_{\bar{Q} A_{1}}(v), I_{\overline{\mathrm{Q}} A_{2}}(v)\right\}, I_{\underline{Q} A_{1} \cup \underline{Q} A_{2}}(v)=\min \left\{I_{\underline{Q} A_{1}}(v), I_{\underline{Q} A_{2}}(v)\right\}, \\
F_{\overline{\mathrm{Q}} A_{1} \cup \bar{Q} A_{2}}(v)=\min \left\{F_{\overline{\mathrm{Q}} A_{1}}(v), F_{\overline{\mathrm{Q}} A_{2}}(v)\right\}, F_{\underline{Q} A_{1} \cup \underline{Q} A_{2}}(v)=\min \left\{F_{\underline{Q} A_{1}}(v), F_{\underline{Q} A_{2}}(v)\right\} .
\end{gathered}
$$

(iv) $\forall v u \in S B_{1}$ but $v u \notin S B_{2}$.

$$
\begin{aligned}
& T_{\overline{\bar{S}} B_{1} \cup \bar{S} B_{2}}(v u)=T_{\bar{S} B_{1}}(v u), T_{\underline{S} B_{1} \cup \underline{S} B_{2}}(v u)=T_{\underline{S} B_{1}}(v u), \\
& I_{\bar{S} B_{1} \cup \bar{S} B_{2}}(v u)=I_{\bar{S} B_{1}}(v u), I_{\underline{S} B_{1} \cup \underline{S_{B}} B_{2}}(v u)=I_{\underline{S} B_{1}}(v u), \\
& F_{\bar{S} B_{1} \cup \bar{S} B_{2}}(v u)=F_{\bar{S} B_{1}}(v u), F_{\underline{S} B_{1} \cup \underline{S} B_{2}}(v u)=F_{\underline{S} B_{1}}(v u) .
\end{aligned}
$$

(v) $\forall v u \notin S B_{1}$ but $v u \in S B_{2}$

$$
\begin{aligned}
& T_{\overline{\bar{S}} B_{1} \cup \bar{S} B_{2}}(v u)=T_{\bar{S} B_{2}}(v u), T_{\underline{S} B_{1} \cup \underline{S} B_{2}}(v u)=T_{\underline{S} B_{2}}(v u), \\
& I_{\bar{S} B_{1} \cup \bar{S} B_{2}}(v u)=I_{\bar{S} B_{2}}(v u), I_{\underline{S} B_{1} \cup \underline{S} B_{2}}(v u)=I_{\underline{S} B_{2}}(v u), \\
& F_{\bar{S} B_{1} \cup \bar{S} B_{2}}(v u)=F_{\bar{S} B_{2}}(v u), F_{\underline{S} B_{1} \cup \underline{S} B_{2}}(v u)=F_{\underline{S} B_{2}}(v u) .
\end{aligned}
$$

(vi) $\forall v u \in S B_{1} \cap \underline{S} B_{2}$

$$
\begin{aligned}
& T_{\bar{S} B_{1} \cup \bar{S} B_{2}}(v u)=\max \left\{T_{\bar{S} B_{1}}(v u), T_{\bar{S} B_{2}}(v u)\right\}, T_{\underline{S} B_{1} \cup \underline{S} B_{2}}(v u)=\max \left\{T_{\underline{S}} B_{1}(v u), T_{\underline{S} B_{2}}(v u)\right\}, \\
& I_{\bar{S} B_{1} \cup \bar{S} B_{2}}(v u)=\min \left\{I_{\bar{S} B_{1}}(v u), I_{\bar{S} B_{2}}(v u)\right\}, I_{\underline{S} B_{1} \cup \underline{S} B_{2}}(v u)=\min \left\{I_{\underline{S} B_{1}}(v u), I_{\underline{S} B_{2}}(v u)\right\}, \\
& F_{\bar{S} B_{1} \cup \bar{S} B_{2}}(v u)=\min \left\{F_{\overline{S_{B}}}(v u), F_{\bar{S} B_{2}}(v u)\right\}, F_{\underline{S} B_{1} \cup \underline{S} B_{2}}(v u)=\min \left\{F_{\underline{S} B_{1}}(v u), F_{\underline{S} B_{2}}(v u)\right\} .
\end{aligned}
$$

Example 5. Let $V=\left\{v_{1}, v_{2}, v_{3}, v_{4}\right\}$ be a set of universes, and $\mathbb{M}=\left\{e_{1}, e_{2}, e_{3}\right\}$ a set of parameters. Then, a neutrosophic soft relation over $V \times \mathbb{M}$ can be written as in Table 7 .

Table 7. Neutrosophic soft relation $Q$.

\begin{tabular}{ccccc}
\hline $\boldsymbol{Q}$ & $\boldsymbol{v}_{\mathbf{1}}$ & $\boldsymbol{v}_{\mathbf{2}}$ & $\boldsymbol{v}_{\mathbf{3}}$ & $\boldsymbol{v}_{\mathbf{4}}$ \\
\hline$e_{1}$ & $(0.5,0.4,0.3)$ & $(0.7,0.6,0.5)$ & $(0.7,0.6,0.4)$ & $(0.5,0.7,0.4)$ \\
$e_{2}$ & $(0.3,0.5,0.6)$ & $(0.4,0.5,0.1)$ & $(0.3,0.6,0.5)$ & $(0.4,0.8,0.2)$ \\
$e_{3}$ & $(0.7,0.5,0.8)$ & $(0.2,0.3,0.8)$ & $(0.7,0.3,0.5)$ & $(0.6,0.4,0.3)$ \\
\hline
\end{tabular}


Let $A_{1}=\left\{\left(e_{1}, 0.5,0.7,0.8\right),\left(e_{2}, 0.7,0.5,0.3\right),\left(e_{3}, 0.4,0.5,0.3\right)\right\}$, and $A_{2}=\left\{\left(e_{1}, 0.6,0.3,0.5\right)\right.$, $\left.\left(e_{2}, 0.5,0.8,0.2\right),\left(e_{3}, 0.5,0.7,0.2\right)\right\}$ are two neutrosophic sets on $\mathbb{M}$, Then, $Q\left(A_{1}\right)=\left(Q\left(A_{1}\right), \bar{Q}\left(A_{1}\right)\right)$ and $Q\left(A_{2}\right)=\left(\underline{Q}\left(A_{2}\right), \bar{Q}\left(A_{2}\right)\right)$ are NSRSs, where

$$
\begin{aligned}
\underline{Q}\left(A_{1}\right) & =\left\{\left(v_{1}, 0.5,0.6,0.5\right),\left(v_{2}, 0.5,0.5,0.7\right)\left(v_{3}, 0.5,0.5,0.7\right),\left(v_{4} 0.4,0.5,0.5\right)\right\}, \\
\overline{\bar{Q}}\left(A_{1}\right) & =\left\{\left(v_{1}, 0.5,0.5,0.6\right),\left(v_{2}, 0.5,0.5,0.3\right),\left(v_{3}, 0.5,0.5,0.5\right),\left(v_{4} 0.5,0.5,0.3\right)\right\}, \\
\underline{Q}\left(A_{2}\right) & =\left\{\left(v_{1}, 0.6,0.5,0.5\right),\left(v_{2}, 0.5,0.7,0.5\right),\left(v_{3}, 0.5,0.7,0.5\right),\left(v_{4}, 0.5,0.6,0.5\right)\right\}, \\
\bar{Q}\left(A_{2}\right) & =\left\{\left(v_{1}, 0.5,0.4,0.5\right),\left(v_{2}, 0.6,0.6,0.2\right),\left(v_{3}, 0.6,0.6,0.5\right),\left(v_{4}, 0.5,0.7,0.2\right)\right\} .
\end{aligned}
$$

Let $E=\left\{v_{1} v_{2}, v_{1} v_{4}, v_{2} v_{2}, v_{2} v_{3}, v_{3} v_{3}, v_{3} v_{4}\right\} \subseteq V \times V$, and $L=\left\{e_{1} e_{2}, e_{1} e_{3}, e_{2} e_{3}\right\} \subset \mathbb{M}$. Then, a neutrosophic soft relation on $E$ can be written as in Table 8.

Table 8. Neutrosophic soft relation $S$.

\begin{tabular}{ccccccc}
\hline$S$ & $v_{1} v_{2}$ & $v_{1} v_{4}$ & $v_{2} v_{2}$ & $v_{2} v_{3}$ & $v_{3} v_{3}$ & $v_{3} v_{4}$ \\
\hline$e_{1} e_{2}$ & $(0.3,0.4,0.1)$ & $(0.4,0.4,0.2)$ & $(0.4,0.5,0.1)$ & $(0.3,0.5,0.4)$ & $(0.3,0.4,0.4)$ & $(0.4,0.5,0.2)$ \\
$e_{1} e_{3}$ & $(0.2,0.3,0.3)$ & $(0.4,0.3,0.2)$ & $(0.2,0.3,0.5)$ & $(0.4,0.3,0.3)$ & $(0.5,0.3,0.3)$ & $(0.5,0.4,0.3)$ \\
$e_{2} e_{3}$ & $(0.2,0.3,0.5)$ & $(0.3,0.3,0.3)$ & $(0.2,0.3,0.1)$ & $(0.4,0.3,0.1)$ & $(0.3,0.3,0.5)$ & $(0.3,0.4,0.3)$ \\
\hline
\end{tabular}

Let $B_{1}=\left\{\left(e_{1} e_{2}, 0.5,0.4,0.5\right),\left(e_{1} e_{3}, 0.3,0.4,0.5\right),\left(e_{2} e_{3}, 0.4,0.4,0.3\right)\right\}$, and $B_{2}=\left\{\left(e_{1} e_{2}, 0.5,0.3,0.2\right)\right.$, $\left.\left(e_{1} e_{3}, 0.4,0.3,0.3\right),\left(e_{2} e_{3}, 0.4,0.6,0.2\right)\right\}$ are two neutrosophic sets on $L$, Then, $S\left(B_{1}\right)=\left(\underline{S}\left(B_{1}\right), \bar{S}\left(B_{1}\right)\right)$ and $S\left(B_{2}\right)=\left(\underline{S}\left(B_{2}\right), \bar{S}\left(B_{2}\right)\right)$ are NSRRs, where

$$
\begin{aligned}
\underline{S}\left(B_{1}\right)= & \left\{\left(v_{1} v_{2}, 0.3,0.4,0.3\right),\left(v_{1} v_{4}, 0.3,0.4,0.4\right),\left(v_{2} v_{2}, 0.4,0.4,0.4\right),\left(v_{2} v_{3}, 0.3,0.4,0.4\right),\right. \\
& \left.\left(v_{3} v_{3}, 0.3,0.4,0.5\right),\left(v_{3} v_{4}, 0.3,0.4,0.5\right)\right\}, \\
\bar{S}\left(B_{1}\right)= & \left\{\left(v_{1} v_{2}, 0.3,0.4,0.5\right),\left(v_{1} v_{4}, 0.4,0.4,0.3\right),\left(v_{2} v_{2}, 0.4,0.4,0.3\right),\left(v_{2} v_{3}, 0.4,0.4,0.3\right),\right. \\
& \left.\left(v_{3} v_{3}, 0.3,0.4,0.5\right),\left(v_{3} v_{4}, 0.4,0.4,0.3\right)\right\} ; \\
\underline{S}\left(B_{2}\right)= & \left\{\left(v_{1} v_{2}, 0.4,0.6,0.2\right),\left(v_{1} v_{4}, 0.4,0.6,0.3\right),\left(v_{2} v_{2}, 0.4,0.6,0.2\right),\left(v_{2} v_{3}, 0.4,0.6,0.3\right),\right. \\
& \left.\left(v_{3} v_{3}, 0.4,0.6,0.3\right),\left(v_{3} v_{4}, 0.4,0.6,0.3\right)\right\}, \\
\bar{S}\left(B_{2}\right)= & \left\{\left(v_{1} v_{2}, 0.3,0.3,0.2\right),\left(v_{1} v_{4}, 0.4,0.3,0.2\right),\left(v_{2} v_{2}, 0.4,0.3,0.2\right),\left(v_{2} v_{3}, 0.4,0.3,0.2\right),\right. \\
& \left.\left(v_{3} v_{3}, 0.4,0.3,0.3\right),\left(v_{3} v_{4}, 0.4,0.4,0.2\right)\right\} .
\end{aligned}
$$

Thus, $G_{1}=\left(\underline{G}_{1}, \bar{G}_{1}\right)$ and $G_{2}=\left(\underline{G}_{2}, \bar{G}_{2}\right)$ are NSRGs, where $\underline{G}_{1}=\left(Q\left(A_{1}\right), \underline{S}\left(B_{1}\right)\right), \bar{G}_{1}=$ $\left(\bar{Q}\left(A_{1}\right), \bar{S}\left(B_{1}\right)\right)$ as shown in Figure 2 .

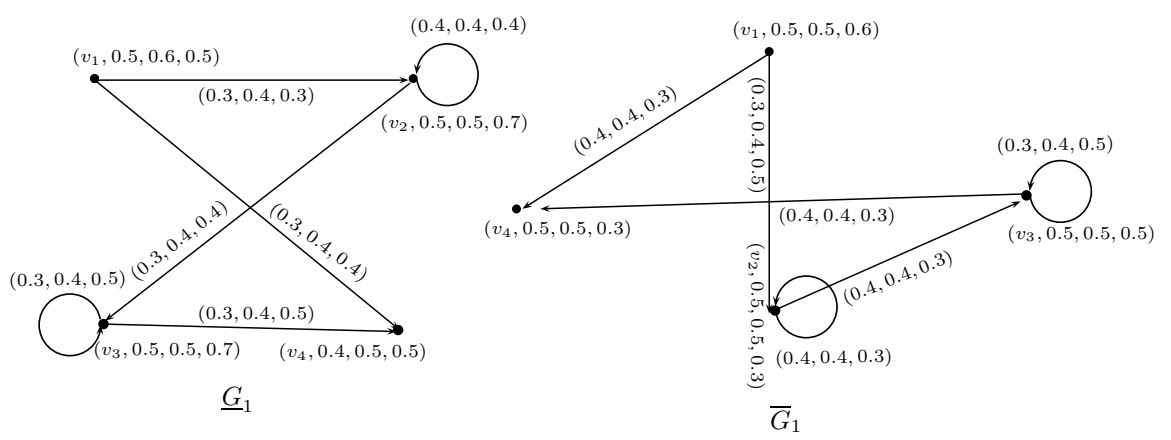

Figure 2. Neutrosophic soft rough graph $G_{1}=\left(\underline{G}_{1}, \bar{G}_{1}\right)$

$\underline{G}_{2}=\left(\underline{Q}\left(A_{2}\right), \underline{S}\left(B_{2}\right)\right), \bar{G}_{2}=\left(\bar{Q}\left(A_{2}\right), \bar{S}\left(B_{2}\right)\right)$ as shown in Figure 3. 


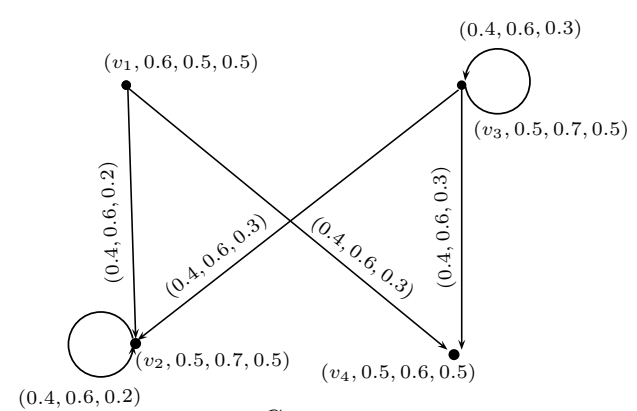

$\underline{G}_{2}$

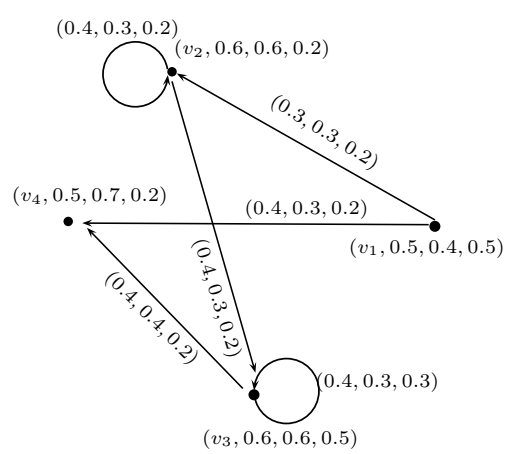

$\bar{G}_{2}$

Figure 3. Neutrosophic soft rough graph $G_{2}=\left(\underline{G}_{2}, \bar{G}_{2}\right)$

The union of $G_{1}=\left(\underline{G}_{1}, \bar{G}_{1}\right)$ and $G_{2}=\left(\underline{G}_{2}, \bar{G}_{2}\right)$ is NSRG $G=G_{1} \cup G_{2}=\left(\underline{G}_{1} \cup \underline{G}_{2}, \bar{G}_{1} \cup \bar{G}_{2}\right)$ as shown in Figure 4.

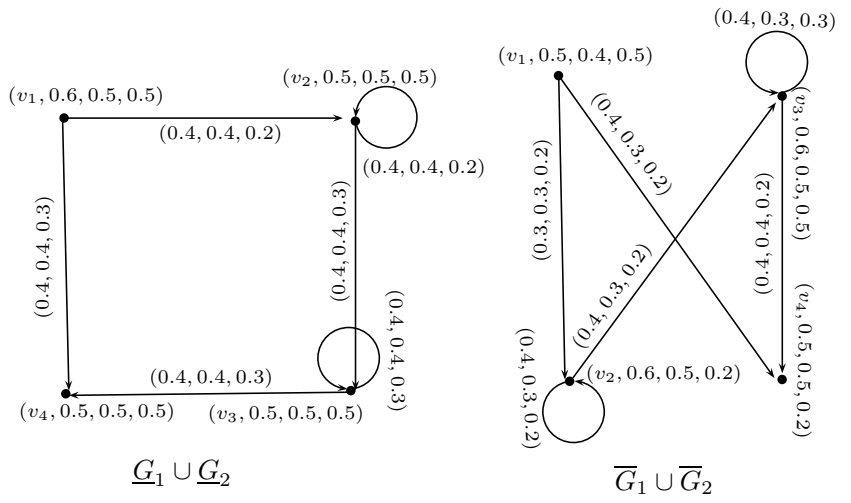

Figure 4. Neutrosophic soft rough graph $G_{1} \cup G_{2}=\left(\underline{G}_{1} \cup \underline{G}_{2}, \bar{G}_{1} \cup \bar{G}_{2}\right)$

Definition 6. Let $G_{1}=\left(\underline{G}_{1}, \bar{G}_{1}\right)$ and $G_{2}=\left(\underline{G}_{2}, \bar{G}_{2}\right)$ be two NSRGs on $V$. The intersection of $G_{1}$ and $G_{2}$ is a neutrosophic soft rough graph $G=G_{1} \cap G_{2}=\left(\underline{G}_{1} \cap \underline{G}_{2}, \bar{G}_{1} \cap \bar{G}_{2}\right)$, where $\underline{G}_{1} \cap \underline{G}_{2}=\left(\underline{Q} A_{1} \cap \underline{Q} A_{2}, \underline{S} B_{1} \cap\right.$ $\left.\underline{S} B_{2}\right)$ and $\bar{G}_{1} \cap \bar{G}_{2}=\left(\bar{Q} A_{1} \cap \bar{Q} A_{2}, \bar{S} B_{1} \cap \bar{S} B_{2}\right)$ are neutrosophic graphs, respectively, such that

(i) $\forall v \in Q A_{1}$ but $v \notin Q A_{2}$.

$$
\begin{gathered}
T_{\bar{Q} A_{1} \cap \bar{Q} A_{2}}(v)=T_{\bar{Q} A_{1}}(v), T_{\underline{Q} A_{1} \cap \underline{Q} A_{2}}(v)=T_{\underline{Q} A_{1}}(v), \\
I_{\bar{Q} A_{1} \cap \bar{Q} A_{2}}(v)=I_{\bar{Q} A_{1}}(v), I_{\underline{Q} A_{1} \cap \underline{Q} A_{2}}(v)=I_{\underline{Q} A_{1}}(v), \\
F_{\bar{Q} A_{1} \cap \bar{Q} A_{2}}(v)=F_{\bar{Q} A_{1}}(v), F_{\underline{Q} A_{1} \cap \underline{Q} A_{2}}(v)=F_{\underline{Q} A_{1}}(v) .
\end{gathered}
$$

(ii) $\forall v \notin Q A_{1}$ but $v \in Q A_{2}$.

$$
\begin{gathered}
T_{\bar{Q} A_{1} \cap \bar{Q} A_{2}}(v)=T_{\bar{Q} A_{2}}(v), T_{\underline{Q} A_{1} \cap \underline{Q} A_{2}}(v)=T_{\underline{Q} A_{2}}(v), \\
I_{\bar{Q} A_{1} \cap \bar{Q} A_{2}}(v)=I_{\bar{Q} A_{2}}(v), I_{\underline{Q} A_{1} \cap \underline{Q} A_{2}}(v)=I_{\underline{Q} A_{2}}(v), \\
F_{\bar{Q} A_{1} \cap \bar{Q} A_{2}}(v)=F_{\bar{Q} A_{2}}(v), F_{\underline{Q} A_{1} \cap \underline{Q} A_{2}}(v)=F_{\underline{Q} A_{2}}(v) .
\end{gathered}
$$


(iii) $\forall v \in Q A_{1} \cap \underline{Q} A_{2}$

$$
\begin{aligned}
T_{\overline{\mathrm{Q}} A_{1} \cap \overline{\mathrm{Q}} A_{2}}(v) & =\min \left\{T_{\overline{\mathrm{Q}} A_{1}}(v), T_{\overline{\mathrm{Q}} A_{2}}(v)\right\}, T_{\underline{Q} A_{1} \cap \underline{Q} A_{2}}(v)=\min \left\{T_{\underline{Q} A_{1}}(v), T_{\underline{Q} A_{2}}(v)\right\}, \\
I_{\overline{\mathrm{Q}} A_{1} \cap \overline{\mathrm{Q}} A_{2}}(v) & =\max \left\{I_{\overline{\mathrm{Q}} A_{1}}(v), I_{\overline{\mathrm{Q}} A_{2}}(v)\right\}, I_{\underline{Q} A_{1} \cap \underline{Q} A_{2}}(v)=\max \left\{I_{\underline{Q} A_{1}}(v), I_{\underline{Q} A_{2}}(v)\right\}, \\
F_{\overline{\mathrm{Q}} A_{1} \cap \bar{Q} A_{2}}(v) & =\max \left\{F_{\overline{\mathrm{Q}} A_{1}}(v), F_{\overline{\mathrm{Q}} A_{2}}(v)\right\}, F_{\underline{Q} A_{1} \cap \underline{Q} A_{2}}(v)=\max \left\{F_{\underline{Q} A_{1}}(v), F_{\underline{Q} A_{2}}(v)\right\} .
\end{aligned}
$$

(iv) $\forall v u \in S B_{1}$ but $v u \notin S B_{2}$.

$$
\begin{aligned}
& T_{\bar{S} B_{1} \cap \bar{S} B_{2}}(v u)=T_{\bar{S} B_{1}}(v u), T_{\underline{S} B_{1} \cap \underline{S} B_{2}}(v u)=T_{\underline{S} B_{1}}(v u), \\
& I_{\bar{S} B_{1} \cap \bar{S} B_{2}}(v u)=I_{\bar{S} B_{1}}(v u), I_{\underline{S} B_{1} \cap \underline{S} B_{2}}(v u)=I_{\underline{S} B_{1}}(v u), \\
& F_{\bar{S} B_{1} \cap \bar{S} B_{2}}(v u)=F_{\bar{S} B_{1}}(v u), F_{\underline{S} B_{1} \cap \underline{S} B_{2}}(v u)=F_{\underline{S} B_{1}}(v u) .
\end{aligned}
$$

(v) $\forall v u \notin S B_{1}$ but $v u \in S B_{2}$

$$
\begin{aligned}
& T_{\bar{S} B_{1} \cap \bar{S} B_{2}}(v u)=T_{\bar{S} B_{2}}(v u), T_{\underline{S} B_{1} \cap \underline{S} B_{2}}(v u)=T_{\underline{S} B_{2}}(v u), \\
& I_{\bar{S} B_{1} \cap \bar{S} B_{2}}(v u)=I_{\bar{S} B_{2}}(v u), I_{\underline{S} B_{1} \cap \underline{S} B_{2}}(v u)=I_{\underline{S} B_{2}}(v u), \\
& F_{\bar{S} B_{1} \cap \bar{S} B_{2}}(v u)=F_{\bar{S} B_{2}}(v u), F_{\underline{S} B_{1} \cap \underline{S} B_{2}}(v u)=F_{\underline{S} B_{2}}(v u) .
\end{aligned}
$$

(vi) $\forall v u \in S B_{1} \cap \underline{S} B_{2}$

$$
\begin{aligned}
& T_{\bar{S} B_{1} \cap \bar{S} B_{2}}(v u)=\min \left\{T_{\bar{S} B_{1}}(v u), T_{\bar{S} B_{2}}(v u)\right\}, T_{\underline{S} B_{1} \cap \underline{S} B_{2}}(v u)=\min \left\{T_{\underline{S}} B_{1}(v u), T_{\underline{S} B_{2}}(v u)\right\}, \\
& I_{\bar{S} B_{1} \cap \bar{S} B_{2}}(v u)=\max \left\{I_{\bar{S} B_{1}}(v u), I_{\bar{S}_{B_{2}}}(v u)\right\}, I_{\underline{S} B_{1} \cap \underline{S} B_{2}}(v u)=\max \left\{I_{\underline{S} B_{1}}(v u), I_{\underline{S} B_{2}}(v u)\right\}, \\
& F_{\overline{S_{B}} \cap \cap \bar{S} B_{2}}(v u)=\max \left\{F_{\bar{S} B_{1}}(v u), F_{\bar{S} B_{2}}(v u)\right\}, F_{\underline{S} B_{1} \cap \underline{S} B_{2}}(v u)=\max \left\{F_{\underline{S} B_{1}}(v u), F_{\underline{S} B_{2}}(v u)\right\} .
\end{aligned}
$$

Definition 7. Let $G_{1}=\left(\underline{G}_{1}, \bar{G}_{1}\right)$ and $G_{2}=\left(\underline{G}_{2}, \bar{G}_{2}\right)$ be two neutrosophic soft rough graphs on $V$. The join of $G_{1}$ and $G_{2}$ is a neutrosophic soft rough graph $G=G_{1}+G_{2}=\left(\underline{G}_{1}+\underline{G}_{2}, \bar{G}_{1}+\bar{G}_{2}\right)$, where $\underline{G}_{1}+\underline{G}_{2}=$ $\left(\underline{Q} A_{1}+\underline{Q} A_{2}, \underline{S} B_{1}+\underline{S} B_{2}\right)$ and $\bar{G}_{1}+\bar{G}_{2}=\left(\bar{Q} A_{1}+\bar{Q} A_{2}, \bar{S} B_{1}+\bar{S} B_{2}\right)$ are neutrosophic graph, respectively, such that

(i) $\forall v \in Q A_{1}$ but $v \notin Q A_{2}$.

$$
\begin{gathered}
T_{\bar{Q} A_{1}+\bar{Q} A_{2}}(v)=T_{\bar{Q} A_{1}}(v), T_{\underline{Q} A_{1}+\underline{Q} A_{2}}(v)=T_{\underline{Q} A_{1}}(v), \\
I_{\bar{Q} A_{1}+\bar{Q} A_{2}}(v)=I_{\bar{Q} A_{1}}(v), I_{\underline{Q} A_{1}+\underline{Q} A_{2}}(v)=I_{\underline{Q} A_{1}}(v), \\
F_{\bar{Q} A_{1}+\bar{Q} A_{2}}(v)=F_{\bar{Q} A_{1}}(v), F_{\underline{Q} A_{1}+\underline{Q} A_{2}}(v)=F_{\underline{Q} A_{1}}(v) .
\end{gathered}
$$

(ii) $\forall v \notin Q A_{1}$ but $v \in Q A_{2}$.

$$
\begin{aligned}
& T_{\overline{\mathrm{Q}} A_{1}+\overline{\mathrm{Q}} A_{2}}(v)=T_{\overline{\mathrm{Q}} A_{2}}(v), T_{\underline{Q} A_{1}+\underline{Q} A_{2}}(v)=T_{\underline{Q} A_{2}}(v), \\
& I_{\overline{\mathrm{Q}} A_{1}+\overline{\mathrm{Q}} A_{2}}(v)=I_{\bar{Q} A_{2}}(v), I_{\underline{Q} A_{1}+\underline{Q} A_{2}}(v)=I_{\underline{Q} A_{2}}(v), \\
& F_{\bar{Q} A_{1}+\bar{Q} A_{2}}(v)=F_{\bar{Q} A_{2}}(v), F_{\underline{Q} A_{1}+\underline{Q} A_{2}}(v)=F_{\underline{Q} A_{2}}(v) .
\end{aligned}
$$

(iii) $\forall v \in Q A_{1} \cap \underline{Q} A_{2}$

$$
\begin{gathered}
T_{\overline{\mathrm{Q}} A_{1}+\overline{\mathrm{Q}} A_{2}}(v)=\max \left\{T_{\overline{\mathrm{Q}} A_{1}}(v), T_{\overline{\mathrm{Q}} A_{2}}(v)\right\}, T_{\underline{Q} A_{1}+\underline{Q} A_{2}}(v)=\max \left\{T_{\underline{Q} A_{1}}(v), T_{\underline{Q} A_{2}}(v)\right\}, \\
I_{\overline{\mathrm{Q}} A_{1}+\overline{\mathrm{Q}} A_{2}}(v)=\min \left\{I_{\overline{\mathrm{Q}} A_{1}}(v), I_{\overline{\mathrm{Q}} A_{2}}(v)\right\}, I_{\underline{Q} A_{1}+\underline{Q} A_{2}}(v)=\min \left\{I_{\underline{Q} A_{1}}(v), I_{\underline{Q} A_{2}}(v)\right\}, \\
F_{\overline{\mathrm{Q}} A_{1}+\overline{\mathrm{Q}} A_{2}}(v)=\min \left\{F_{\overline{\mathrm{Q}} A_{1}}(v), F_{\overline{\mathrm{Q}} A_{2}}(v)\right\}, F_{\underline{Q} A_{1}+\underline{Q} A_{2}}(v)=\min \left\{F_{\underline{Q} A_{1}}(v), F_{\underline{Q} A_{2}}(v)\right\} .
\end{gathered}
$$


(iv) $\forall v u \in S B_{1}$ but $v u \notin S B_{2}$.

$$
\begin{aligned}
& T_{\bar{S} B_{1}+\bar{S} B_{2}}(v u)=T_{\bar{S} B_{1}}(v u), T_{\underline{S} B_{1}+\underline{S} B_{2}}(v u)=T_{\underline{S} B_{1}}(v u), \\
& I_{\bar{S} B_{1}+\bar{S} B_{2}}(v u)=I_{\bar{S} B_{1}}(v u), I_{\underline{S} B_{1}+\underline{S} B_{2}}(v u)=I_{\underline{S} B_{1}}(v u), \\
& F_{\bar{S} B_{1}+\bar{S} B_{2}}(v u)=F_{\bar{S} B_{1}}(v u), F_{\underline{S} B_{1}+\underline{S} B_{2}}(v u)=F_{\underline{S} B_{1}}(v u) .
\end{aligned}
$$

(v) $\forall v u \notin S B_{1}$ but $v u \in S B_{2}$

$$
\begin{aligned}
T_{\bar{S} B_{1}+\bar{S} B_{2}}(v u) & =T_{\bar{S} B_{2}}(v u), T_{\underline{S} B_{1}+\underline{S} B_{2}}(v u)=T_{\underline{S} B_{2}}(v u), \\
I_{\bar{S} B_{1}+\bar{S} B_{2}}(v u) & =I_{\bar{S} B_{2}}(v u), I_{\underline{S} B_{1}+\underline{S} B_{2}}(v u)=I_{\underline{S} B_{2}}(v u), \\
F_{\bar{S} B_{1}+\bar{S} B_{2}}(v u) & =F_{\bar{S} B_{2}}(v u), F_{\underline{S} B_{1}+\underline{S} B_{2}}(v u)=F_{\underline{S} B_{2}}(v u) .
\end{aligned}
$$

(vi) $\forall v u \in S B_{1} \cap \underline{S} B_{2}$

$$
\begin{aligned}
& T_{\bar{S} B_{1}+\bar{S} B_{2}}(v u)=\max \left\{T_{\bar{S} B_{1}}(v u), T_{\bar{S} B_{2}}(v u)\right\}, T_{\underline{S} B_{1}+\underline{S} B_{2}}(v u)=\max \left\{T_{\underline{S}} B_{1}(v u), T_{\underline{S} B_{2}}(v u)\right\}, \\
& I_{\bar{S} B_{1}+\bar{S} B_{2}}(v u)=\min \left\{I_{\bar{S} B_{1}}(v u), I_{\bar{S} B_{2}}(v u)\right\}, I_{\underline{S} B_{1}+\underline{S} B_{2}}(v u)=\min \left\{I_{\underline{S} B_{1}}(v u), I_{\underline{S} B_{2}}(v u)\right\}, \\
& F_{\bar{S} B_{1}+\bar{S} B_{2}}(v u)=\min \left\{F_{\bar{S} B_{1}}(v u), F_{\bar{S} B_{2}}(v u)\right\}, F_{\underline{S} B_{1}+\underline{S} B_{2}}(v u)=\min \left\{F_{\underline{S} B_{1}}(v u), F_{\underline{S} B_{2}}(v u)\right\} .
\end{aligned}
$$

(vii) $\forall v u \in \tilde{E}$, where $\tilde{E}$ is the set of edges joining vertices of $Q A_{1}$ and $Q A_{2}$.

$$
\begin{aligned}
& T_{\bar{S} B_{1}+\bar{S} B_{2}}(v u)=\min \left\{T_{\overline{\mathrm{Q}} A_{1}}(v), T_{\overline{\mathrm{Q}} A_{2}}(u)\right\}, T_{\underline{S} B_{1}+\underline{S} B_{2}}(v u)=\min \left\{T_{\underline{Q} A_{1}}(v), T_{\underline{Q} A_{2}}(u)\right\}, \\
& I_{\bar{S} B_{1}+\bar{S} B_{2}}(v u)=\max \left\{I_{\bar{Q} A_{1}}(v), I_{\bar{Q} A_{2}}(u)\right\}, I_{\underline{S} B_{1}+\underline{S} B_{2}}(v u)=\max \left\{I_{\underline{Q} A_{1}}(v), I_{\underline{Q} A_{2}}(u)\right\}, \\
& F_{\bar{S} B_{1}+\bar{S} B_{2}}(v u)=\max \left\{F_{\bar{Q} A_{1}}(v), F_{\bar{Q} A_{2}}(u)\right\}, F_{\underline{S} B_{1}+\underline{S} B_{2}}(v u)=\max \left\{F_{\underline{Q} A_{1}}(v), F_{\underline{Q} A_{2}}(u)\right\} .
\end{aligned}
$$

Definition 8. The Cartesian product of $G_{1}$ and $G_{2}$ is a $G=G_{1} \ltimes G_{2}=\left(\underline{G}_{1} \ltimes \underline{G}_{2}, \bar{G}_{1} \ltimes \bar{G}_{2}\right)$, where $\underline{G}_{1} \ltimes \underline{G}_{2}=$ $\left(\underline{Q} A_{1} \ltimes \underline{Q} A_{2}, \underline{S} B_{1} \ltimes \underline{S} B_{2}\right)$ and $\bar{G}_{1} \ltimes \bar{G}_{2}=\left(\bar{Q} A_{1} \ltimes \bar{Q} A_{2}, \bar{S} B_{1} \ltimes \bar{S} B_{2}\right)$ are neutrosophic digraph, such that

(i) $\forall\left(v_{1}, v_{2}\right) \in Q A_{1} \times Q A_{2}$.

$$
\begin{aligned}
& T_{\left(\overline{\mathrm{Q}} A_{1} \ltimes \overline{\mathrm{Q}} A_{2}\right)}\left(v_{1}, v_{2}\right)=\min \left\{T_{\overline{\mathrm{Q}} A_{1}}\left(v_{1}\right), T_{\overline{\mathrm{Q}} A_{2}}\left(v_{1}\right)\right\}, T_{\left(\underline{\mathrm{Q} A} A_{1} \ltimes \underline{Q} A_{2}\right)}\left(v_{1}, v_{2}\right)=\min \left\{T_{\underline{Q} A_{1}}\left(v_{1}\right), T_{\underline{Q} A_{2}}\left(v_{1}\right)\right\}, \\
& I_{\left(\overline{\mathrm{Q}} A_{1} \ltimes \bar{Q} A_{2}\right)}\left(v_{1}, v_{2}\right)=\max \left\{I_{\overline{\mathrm{Q}} A_{1}}\left(v_{1}\right), I_{\overline{\mathrm{Q}} A_{2}}\left(v_{1}\right)\right\}, I_{\left(\underline{Q} A_{1} \ltimes \underline{Q} A_{2}\right)}\left(v_{1}, v_{2}\right)=\max \left\{I_{\underline{Q} A_{1}}\left(v_{1}\right), I_{\underline{Q} A_{2}}\left(v_{1}\right)\right\}, \\
& F_{\left(\overline{\mathrm{Q}} A_{1} \ltimes \bar{Q} A_{2}\right)}\left(v_{1}, v_{2}\right)=\max \left\{F_{\overline{\mathrm{Q}} A_{1}}\left(v_{1}\right), F_{\overline{\mathrm{Q}} A_{2}}\left(v_{1}\right)\right\}, F_{\left(\underline{Q} A_{1} \ltimes \underline{Q} A_{2}\right)}\left(v_{1}, v_{2}\right)=\max \left\{F_{\underline{Q} A_{1}}\left(v_{1}\right), F_{\underline{Q} A_{2}}\left(v_{1}\right)\right\} .
\end{aligned}
$$

(ii) $\forall v_{1} v_{2} \in S B_{2}, v \in Q A_{1}$.

$$
\begin{aligned}
& T_{\left(\bar{S} B_{1} \ltimes \bar{S} B_{2}\right)}\left(\left(v, v_{1}\right)\left(v, v_{2}\right)\right)=\min \left\{T_{\bar{Q} A_{1}}(v), T_{\bar{S} B_{2}}\left(v_{1} v_{2}\right)\right\}, \\
& T_{\left(\underline{S} B_{1} \ltimes \underline{S} B_{2}\right)}\left(\left(v, v_{1}\right)\left(v, v_{2}\right)\right)=\min \left\{T_{\underline{Q} A_{1}}(v), T_{\underline{S} B_{2}}\left(v_{1} v_{2}\right)\right\}, \\
& I_{\left(\bar{S} B_{1} \ltimes \bar{S} B_{2}\right)}\left(\left(v, v_{1}\right)\left(v, v_{2}\right)\right)=\max \left\{I_{\bar{Q} A_{1}}(v), I_{\bar{S} B_{2}}\left(v_{1} v_{2}\right)\right\}, \\
& I_{\left(\underline{S} B_{1} \ltimes \underline{S} B_{2}\right)}\left(\left(v, v_{1}\right)\left(v, v_{2}\right)\right)=\max \left\{I_{\underline{Q} A_{1}}(v), I_{\underline{S} B_{2}}\left(v_{1} v_{2}\right)\right\}, \\
& F_{\left(\bar{S} B_{1} \ltimes \bar{S} B_{2}\right)}\left(\left(v, v_{1}\right)\left(v, v_{2}\right)\right)=\max \left\{F_{\bar{Q} A_{1}}(v), F_{\bar{S} B_{2}}\left(v_{1} v_{2}\right)\right\}, \\
& F_{\left(\underline{S} B_{1} \ltimes \underline{S} B_{2}\right)}\left(\left(v, v_{1}\right)\left(v, v_{2}\right)\right)=\max \left\{F_{\underline{Q} A_{1}}(v), F_{\underline{S} B_{2}}\left(v_{1} v_{2}\right)\right\} .
\end{aligned}
$$


(iii) $\forall v_{1} v_{2} \in S B_{1}, v \in Q A_{2}$.

$$
\begin{aligned}
& T_{\left(\underline{\left.\underline{S} B_{1} \ltimes \underline{S} B_{2}\right)}\right.}\left(\left(v_{1}, v\right)\left(v_{2}, v\right)\right)=\min \left\{T_{\underline{\underline{S} B_{1}}}\left(v_{1} v_{2}\right), T_{\underline{Q} A_{2}}(v)\right\}, \\
& T_{\left(\bar{S} B_{1} \ltimes \bar{S} B_{2}\right)}\left(\left(v_{1}, v\right)\left(v_{2}, v\right)\right)=\min \left\{T_{\bar{S} B_{1}}\left(v_{1} v_{2}\right), T_{\bar{Q} A_{2}}(v)\right\}, \\
& I_{\left(\bar{S} B_{1} \ltimes \bar{S} B_{2}\right)}\left(\left(v_{1}, v\right)\left(v_{2}, v\right)\right)=\max \left\{I_{\bar{S} B_{1}}\left(v_{1} v_{2}\right), I_{\bar{Q} A_{2}}(v)\right\}, \\
& I_{\left(\underline{S} B_{1} \ltimes \underline{S} B_{2}\right)}\left(\left(v_{1}, v\right)\left(v_{2}, v\right)\right)=\max \left\{I_{\underline{S}_{1}}\left(v_{1} v_{2}\right), I_{\underline{Q} A_{2}}(v)\right\}, \\
& F_{\left(\bar{S} B_{1} \ltimes \bar{S} B_{2}\right)}\left(\left(v_{1}, v\right)\left(v_{2}, v\right)\right)=\max \left\{F_{\bar{S} B_{1}}\left(v_{1} v_{2}\right), F_{\bar{Q} A_{2}}(v)\right\}, \\
& F_{\left(\underline{S} B_{1} \ltimes \underline{S} B_{2}\right)}\left(\left(v_{1}, v\right)\left(v_{2}, v\right)\right)=\max \left\{F_{\underline{S} B_{1}}\left(v_{1} v_{2}\right), F_{\underline{Q} A_{2}}(v)\right\} .
\end{aligned}
$$

Definition 9. The cross product of $G_{1}$ and $G_{2}$ is a neutrosophic soft rough graph $G=G_{1} \odot G_{2}=\left(\underline{G}_{1} \odot\right.$ $\left.\underline{G}_{2}, \bar{G}_{1} \odot \bar{G}_{2}\right)$, where $\underline{G}_{1} \odot \underline{G}_{2}=\left(\underline{Q} A_{1} \odot \underline{Q} A_{2}, \underline{S} B_{1} \odot \underline{S} B_{2}\right)$ and $\bar{G}_{1} \odot \bar{G}_{2}=\left(\bar{Q} A_{1} \odot \bar{Q} A_{2}, \bar{S} B_{1} \odot \bar{S} B_{2}\right)$ are neutrosophic graphs, respectively, such that

(i) $\forall\left(v_{1}, v_{2}\right) \in Q A_{1} \times Q A_{2}$.

$$
\begin{aligned}
& T_{\left(\overline{\mathrm{Q}} A_{1} \odot \overline{\mathrm{Q}} A_{2}\right)}\left(v_{1}, v_{2}\right)=\min \left\{T_{\overline{\mathrm{Q}} A_{1}}\left(v_{1}\right), T_{\overline{\mathrm{Q}} A_{2}}\left(v_{1}\right)\right\}, T_{\left(\underline{\mathrm{Q}} A_{1} \odot \underline{Q} A_{2}\right)}\left(v_{1}, v_{2}\right)=\min \left\{T_{\underline{\mathrm{Q}} A_{1}}\left(v_{1}\right), T_{\underline{Q} A_{2}}\left(v_{1}\right)\right\}, \\
& I_{\left(\overline{\mathrm{Q}} A_{1} \odot \overline{\mathrm{Q}} A_{2}\right)}\left(v_{1}, v_{2}\right)=\max \left\{I_{\overline{\mathrm{Q}} A_{1}}\left(v_{1}\right), I_{\overline{\mathrm{Q}} A_{2}}\left(v_{1}\right)\right\}, I_{\left(\underline{\mathrm{Q}} A_{1} \odot \underline{Q} A_{2}\right)}\left(v_{1}, v_{2}\right)=\max \left\{I_{\underline{Q} A_{1}}\left(v_{1}\right), I_{\mathrm{Q} A_{2}}\left(v_{1}\right)\right\}, \\
& F_{\left(\overline{\mathrm{Q}} A_{1} \odot \overline{\mathrm{Q}} A_{2}\right)}\left(v_{1}, v_{2}\right)=\max \left\{F_{\overline{\mathrm{Q}} A_{1}}\left(v_{1}\right), F_{\overline{\mathrm{Q}} A_{2}}\left(v_{1}\right)\right\}, F_{\left(\underline{Q} A_{1} \odot \underline{Q} A_{2}\right)}\left(v_{1}, v_{2}\right)=\max \left\{F_{\underline{Q} A_{1}}\left(v_{1}\right), F_{\underline{Q} A_{2}}\left(v_{1}\right)\right\} .
\end{aligned}
$$

(ii) $\forall v_{1} u_{1} \in S B_{1}, v_{2} u_{2} \in S B_{2}$.

$$
\begin{aligned}
T_{\left(\bar{S} B_{1} \odot \bar{S} B_{2}\right)}\left(\left(v_{1}, v_{2}\right)\left(u_{1}, u_{2}\right)\right) & =\min \left\{T_{\bar{S} B_{1}}\left(v_{1} u_{1}\right), T_{\bar{S} B_{2}}\left(v_{1} u_{2}\right)\right\}, \\
T_{\left(\underline{S} B_{1} \odot \underline{S} B_{2}\right)}\left(\left(v_{1}, v_{2}\right)\left(u_{1}, u_{2}\right)\right) & =\min \left\{T_{\underline{S} B_{1}}\left(v_{1} u_{1}\right), T_{\underline{S} B_{2}}\left(v_{1} u_{2}\right)\right\}, \\
I_{\left(\bar{S} B_{1} \odot \bar{S} B_{2}\right.}\left(\left(v_{1}, v_{2}\right)\left(u_{1}, u_{2}\right)\right) & =\max \left\{I_{\bar{S} B_{1}}\left(v_{1} u_{1}\right), I_{\bar{S} B_{2}}\left(v_{1} u_{2}\right)\right\}, \\
I_{\left(\underline{S} B_{1} \odot \underline{S} B_{2}\right)}\left(\left(v_{1}, v_{2}\right)\left(u_{1}, u_{2}\right)\right) & =\max \left\{I_{\underline{I}_{B_{1}}}\left(v_{1} u_{1}\right), I_{\underline{S} B_{2}}\left(v_{1} u_{2}\right)\right\}, \\
F_{\left(\bar{S} B_{1} \odot \bar{S} B_{2}\right.}\left(\left(v_{1}, v_{2}\right)\left(u_{1}, u_{2}\right)\right) & =\max \left\{F_{\bar{S} B_{1}}\left(v_{1} u_{1}\right), F_{\bar{S} B_{2}}\left(v_{1} u_{2}\right)\right\}, \\
F_{\left(\underline{S} B_{1} \odot \underline{S} B_{2}\right)}\left(\left(v_{1}, v_{2}\right)\left(u_{1}, u_{2}\right)\right) & =\max \left\{F_{\underline{S} B_{1}}\left(v_{1} u_{1}\right), F_{\underline{S} B_{2}}\left(v_{1} u_{2}\right)\right\} .
\end{aligned}
$$

Definition 10. The rejection of $G_{1}$ and $G_{2}$ is a neutrosophic soft rough graph $G=G_{1} \mid G_{2}=\left(\underline{G}_{1}\left|\underline{G}_{2}, \bar{G}_{1}\right| \bar{G}_{2}\right)$, where $\underline{G}_{1} \mid \underline{G}_{2}=\left(\underline{S} A_{1}\left|\underline{S} A_{2}, \underline{S} B_{1}\right| \underline{S} B_{2}\right)$ and $\bar{G}_{1} \mid \bar{G}_{2}=\left(\bar{S} A_{1}\left|\bar{S} A_{2}, \bar{S} B_{1}\right| \bar{S} B_{2}\right)$ are neutrosophic graphs such that

(i) $\forall\left(v_{1}, v_{2}\right) \in Q A_{1} \times Q A_{2}$.

$$
\begin{aligned}
& T_{\left(\overline{\mathrm{Q}} A_{1} \mid \overline{\mathrm{Q}} A_{2}\right)}\left(v_{1}, v_{2}\right)=\min \left\{T_{\overline{\mathrm{Q}} A_{1}}\left(v_{1}\right), T_{\overline{\mathrm{Q}} A_{2}}\left(v_{2}\right)\right\}, T_{\left(\underline{Q} A_{1} \mid \underline{Q} A_{2}\right)}\left(v_{1}, v_{2}\right)=\min \left\{T_{\underline{Q} A_{1}}\left(v_{1}\right), T_{\underline{Q} A_{2}}\left(v_{2}\right)\right\}, \\
& I_{\left(\overline{\mathrm{Q}} A_{1} \mid \overline{\mathrm{Q}} A_{2}\right)}\left(v_{1}, v_{2}\right)=\max \left\{I_{\overline{\mathrm{Q}} A_{1}}\left(v_{1}\right), I_{\overline{\mathrm{Q}} A_{2}}\left(v_{2}\right)\right\}, I_{\left(\underline{Q} A_{1} \mid \underline{Q} A_{2}\right)}\left(v_{1}, v_{2}\right)=\max \left\{I_{\underline{Q} A_{1}}\left(v_{1}\right), I_{\underline{Q} A_{2}}\left(v_{2}\right)\right\}, \\
& F_{\left(\overline{\mathrm{Q}} A_{1} \mid \overline{\mathrm{Q}} A_{2}\right)}\left(v_{1}, v_{2}\right)=\max \left\{F_{\overline{\mathrm{Q}} A_{1}}\left(v_{1}\right), F_{\overline{\mathrm{Q}} A_{2}}\left(v_{2}\right)\right\}, F_{\left(\underline{Q} A_{1} \mid \underline{Q} A_{2}\right)}\left(v_{1}, v_{2}\right)=\max \left\{F_{\underline{Q} A_{1}}\left(v_{1}\right), F_{\underline{Q} A_{2}}\left(v_{2}\right)\right\} .
\end{aligned}
$$

(ii) $\forall v_{2} u_{2} \notin S B_{2}, v \in Q A_{1}$.

$$
\begin{aligned}
T_{\left(\bar{S} B_{1} \mid \bar{S} B_{2}\right)}\left(\left(v, v_{2}\right)\left(v, u_{2}\right)\right) & =\min \left\{T_{\bar{Q} A_{1}}(v), T_{\bar{Q} A_{2}}\left(v_{2}\right), T_{\bar{Q} A_{2}}\left(u_{2}\right)\right\}, \\
T_{\left(\underline{S} B_{1} \mid \underline{Q} B_{2}\right)}\left(\left(v, v_{2}\right)\left(v, u_{2}\right)\right) & =\min \left\{T_{\underline{Q} A_{1}}(v), T_{\underline{Q} A_{2}}\left(v_{2}\right), T_{\underline{Q} A_{2}}\left(u_{2}\right)\right\}, \\
\left(I_{\left.\bar{S} B_{1} \mid \bar{S} B_{2}\right)}\left(\left(v, v_{2}\right)\left(v, u_{2}\right)\right)\right. & =\max \left\{I_{\bar{Q} A_{1}}(v), I_{\bar{Q} A_{2}}\left(v_{2}\right), I_{\bar{Q} A_{2}}\left(u_{2}\right)\right\}, \\
\left(I_{\left.\underline{S} B_{1} \mid \underline{S} B_{2}\right)}\left(\left(v, v_{2}\right)\left(v, u_{2}\right)\right)\right. & =\max \left\{I_{\underline{Q} A_{1}}(v), I_{\underline{Q} A_{2}}\left(v_{2}\right), I_{\underline{Q} A_{2}}\left(u_{2}\right)\right\}, \\
\left(F_{\left.\bar{S} B_{1} \mid \bar{S} B_{2}\right)}\left(\left(v, v_{2}\right)\left(v, u_{2}\right)\right)\right. & =\max \left\{F_{\bar{Q} A_{1}}(v), F_{\bar{Q} A_{2}}\left(v_{2}\right), F_{\bar{Q} A_{2}}\left(u_{2}\right)\right\}, \\
\left(F_{\left.\underline{S} B_{1} \mid \underline{S} B_{2}\right)}\left(\left(v, v_{2}\right)\left(v, u_{2}\right)\right)\right. & =\max \left\{F_{\underline{Q} A_{1}}(v), F_{Q} A_{2}\left(v_{2}\right), F_{\underline{Q} A_{2}}\left(u_{2}\right)\right\} .
\end{aligned}
$$


(iii) $\forall v_{1} u_{1} \notin S B_{1}, v \in Q A_{2}$,

$$
\begin{aligned}
& T_{\left(\underline{S} B_{1} \mid \underline{S} B_{2}\right)}\left(\left(v_{1}, v\right)\left(u_{1}, v\right)\right)=\min \left\{T_{\underline{Q} A_{1}}\left(v_{1}\right), T_{\underline{Q} A_{1}}\left(u_{1}\right), T_{\underline{Q} A_{2}}(v)\right\}, \\
& I_{\left(\underline{S} B_{1} \mid \underline{S} B_{2}\right)}\left(\left(v_{1}, v\right)\left(u_{1}, v\right)\right)=\max \left\{I_{\underline{Q} A_{1}}\left(v_{1}\right), I_{\underline{Q} A_{1}}\left(u_{1}\right), I_{\underline{Q} A_{2}}(v)\right\}, \\
& F_{\left(\underline{S} B_{1} \mid \underline{S} B_{2}\right)}\left(\left(v_{1}, v\right)\left(u_{1}, v\right)\right)=\max \left\{F_{\underline{Q} A_{1}}\left(v_{1}\right), F_{\underline{Q} A_{1}}\left(u_{1}\right), F_{\underline{Q} A_{2}}(v)\right\}, \\
& T_{\left(\bar{S} B_{1} \mid \bar{S} B_{2}\right)}\left(\left(v_{1}, v\right)\left(u_{1}, v\right)\right)=\min \left\{T_{\bar{Q} A_{1}}\left(v_{1}\right), T_{\bar{Q} A_{1}}\left(u_{1}\right), T_{\bar{Q} A_{2}}(v)\right\}, \\
& I_{\left(\bar{S} B_{1} \mid \bar{S} B_{2}\right)}\left(\left(v_{1}, v\right)\left(u_{1}, v\right)\right)=\max \left\{I_{\bar{Q} A_{1}}\left(v_{1}\right), I_{\bar{Q} A_{1}}\left(u_{1}\right), I_{\bar{Q} A_{2}}(v)\right\}, \\
& F_{\left(\bar{S} B_{1} \mid \bar{S} B_{2}\right)}\left(\left(v_{1}, v\right)\left(u_{1}, v\right)\right)=\max \left\{F_{\bar{Q} A_{1}}\left(v_{1}\right), F_{\bar{Q} A_{1}}\left(u_{1}\right), F_{\bar{Q} A_{2}}(v)\right\} .
\end{aligned}
$$

(iv) $\forall v_{1} u_{1} \notin \bar{S} B_{1}, v_{2} u_{2} \notin \bar{S} B_{2}, v_{1}=u_{1}$.

$$
\begin{aligned}
& T_{\left(\underline{\left.\underline{S} B_{1} \mid \underline{S} B_{2}\right)}\right.}\left(\left(v_{1}, v_{2}\right)\left(u_{1}, u_{2}\right)\right)=\min \left\{T_{\underline{Q} A_{1}}\left(v_{1}\right), T_{\underline{Q} A_{1}}\left(u_{1}\right), T_{\underline{Q} A_{2}}\left(v_{2}\right), T_{\underline{Q} A_{2}}\left(u_{2}\right)\right\}, \\
& I_{\left(\underline{S} B_{1} \mid \underline{S} B_{2}\right)}\left(\left(v_{1}, v_{2}\right)\left(u_{1}, u_{2}\right)\right)=\max \left\{I_{\underline{Q} A_{1}}\left(v_{1}\right), I_{\underline{Q} A_{1}}\left(u_{1}\right), I_{\underline{Q} A_{2}}\left(v_{2}\right), I_{\underline{Q} A_{2}}\left(u_{2}\right)\right\}, \\
& F_{\left(\underline{S} B_{1} \mid \underline{S} B_{2}\right)}\left(\left(v_{1}, v_{2}\right)\left(u_{1}, u_{2}\right)\right)=\max \left\{F_{\underline{Q} A_{1}}\left(v_{1}\right), F_{\underline{Q} A_{1}}\left(u_{1}\right), F_{\underline{Q} A_{2}}\left(v_{2}\right), F_{\underline{Q} A_{2}}\left(u_{2}\right)\right\}, \\
& T_{\left(\bar{S} B_{1} \mid \bar{S} B_{2}\right)}\left(\left(v_{1}, v_{2}\right)\left(u_{1}, u_{2}\right)\right)=\min \left\{T_{\bar{Q} A_{1}}\left(v_{1}\right), T_{\bar{Q} A_{1}}\left(u_{1}\right), T_{\bar{Q} A_{2}}\left(v_{2}\right), T_{\bar{Q} A_{2}}\left(u_{2}\right)\right\}, \\
& I_{\left(\bar{S} B_{1} \mid \bar{S} B_{2}\right)}\left(\left(v_{1}, v_{2}\right)\left(u_{1}, u_{2}\right)\right)=\max \left\{I_{\bar{Q} A_{1}}\left(v_{1}\right), I_{\bar{Q} A_{1}}\left(u_{1}\right), I_{\bar{Q} A_{2}}\left(v_{2}\right), I_{\bar{Q} A_{2}}\left(u_{2}\right)\right\}, \\
& F_{\left(\bar{S} B_{1} \mid \bar{S} B_{2}\right)}\left(\left(v_{1}, v_{2}\right)\left(u_{1}, u_{2}\right)\right)=\max \left\{F_{\overline{\mathrm{Q}} A_{1}}\left(v_{1}\right), F_{\overline{\mathrm{Q}} A_{1}}\left(u_{1}\right), F_{\bar{Q} A_{2}}\left(v_{2}\right), F_{\overline{\mathrm{Q}} A_{2}}\left(u_{2}\right)\right\},
\end{aligned}
$$

Example 6. Let $G_{1}=\left(\underline{G}_{1}, \bar{G}_{1}\right)$ and $G_{2}=\left(\underline{G}_{2}, \bar{G}_{2}\right)$ be two neutrosophic soft rough graphs on $V$, where $\underline{G}_{1}=\left(Q A_{1}, \underline{S} B_{1}\right)$ and $\bar{G}_{1}=\left(\bar{Q} A_{1}, \bar{S} B_{1}\right)$ are neutrosophic graphs as shown in Figure 2 and $\underline{G}_{2}=\left(Q A_{2}, \underline{S} B_{2}\right)$ and $\bar{G}_{2}=\left(\bar{Q} A_{2}, \bar{S} B_{2}\right)$ are neutrosophic graphs as shown in Figure 3. The Cartesian product of $G_{1}=\left(\underline{G}_{1}, \bar{G}_{1}\right)$ and $G_{2}=\left(\underline{G}_{2}, \bar{G}_{2}\right)$ is NSRG $G=G_{1} \times G_{2}=\left(\underline{G}_{1} \times \underline{G}_{2}, \bar{G}_{1} \times \bar{G}_{2}\right)$ as shown in Figure 5 .

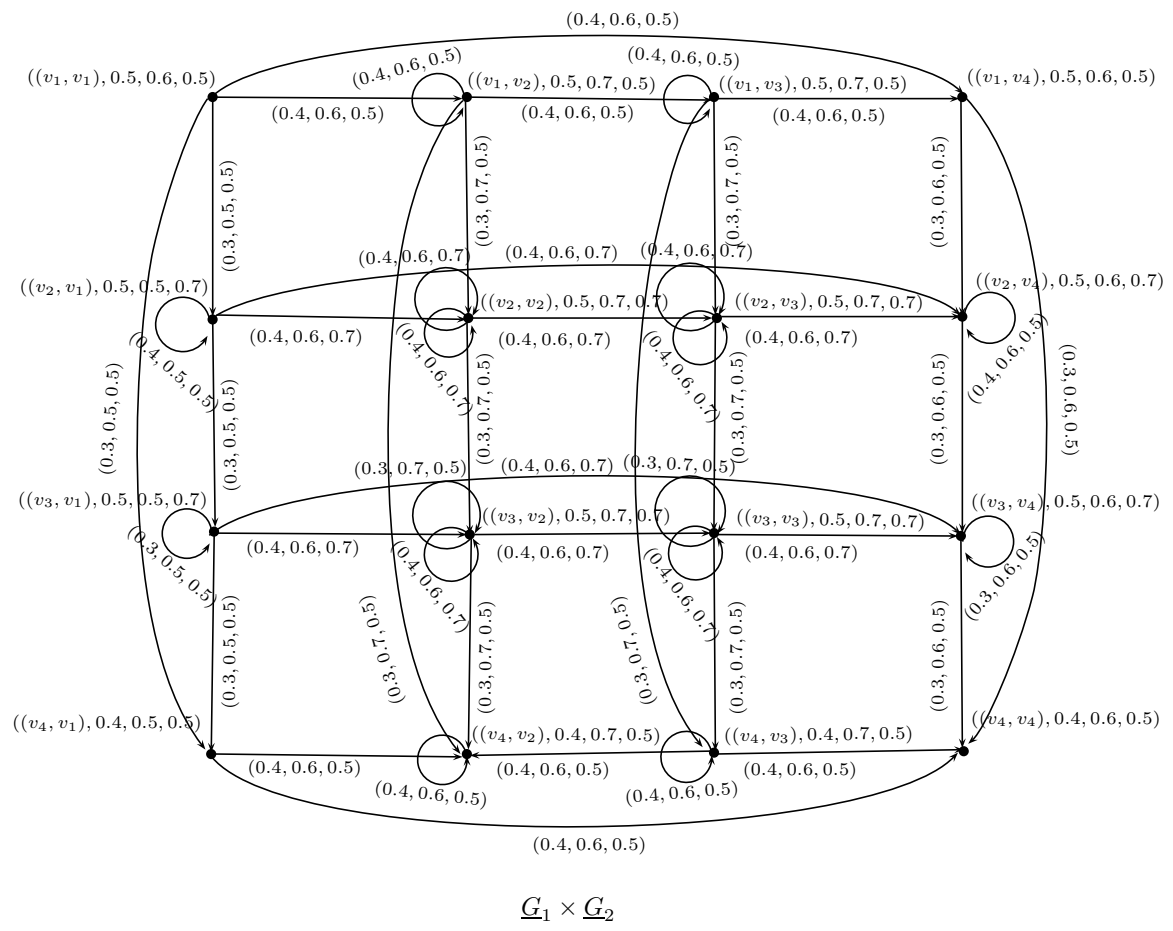

(a)

Figure 5. Cont. 


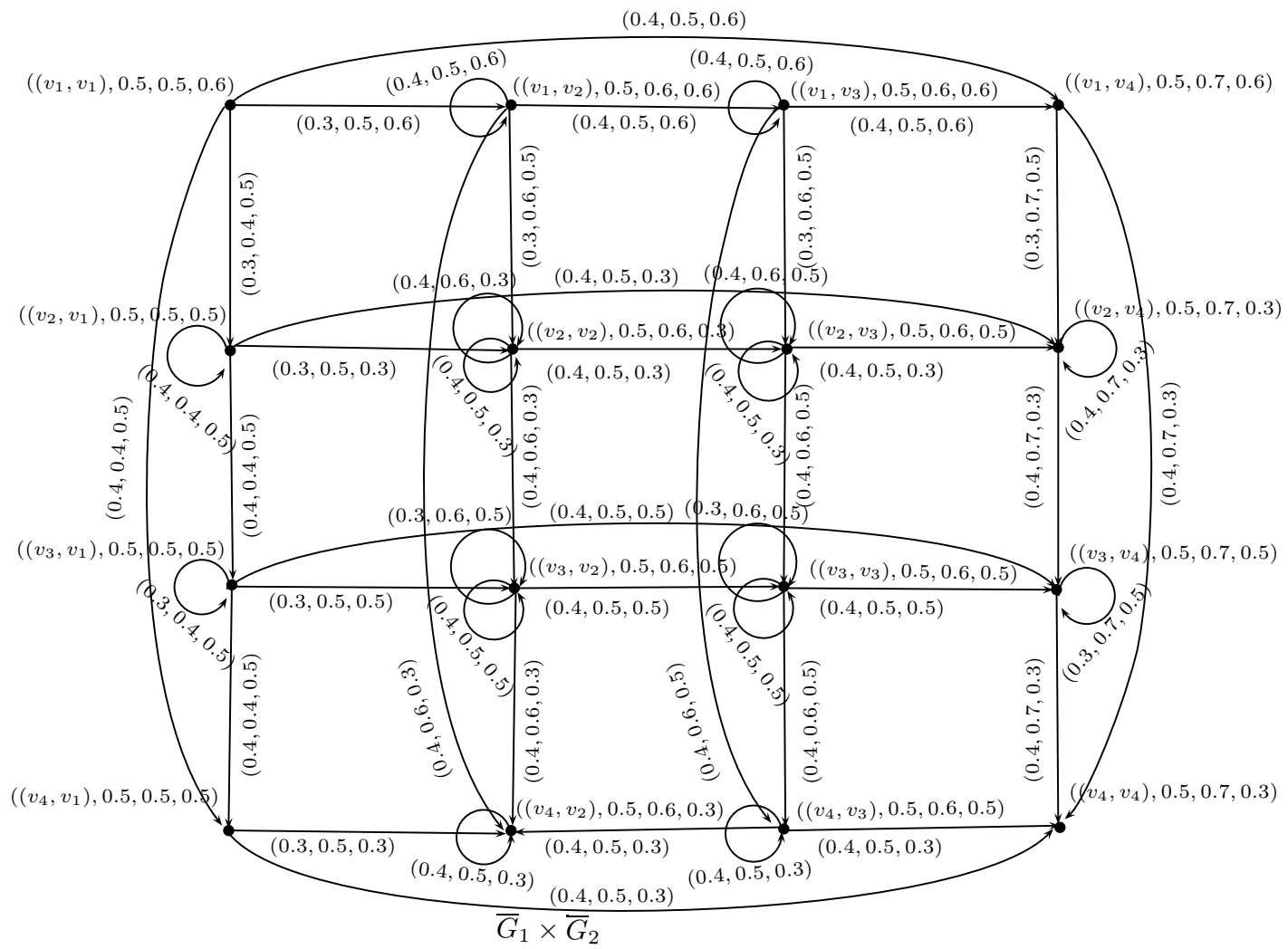

(b)

Figure 5. Cartesian product of two neutrosophic soft rough graphs $G_{1} \times G_{2}$

Definition 11. The symmetric difference of $G_{1}$ and $G_{2}$ is a neutrosophic soft rough graph $G=G_{1} \oplus G_{2}=$ $\left(\underline{G}_{1} \oplus \underline{G}_{2}, \bar{G}_{1} \oplus \bar{G}_{2}\right)$, where $\underline{G}_{1} \oplus \underline{G}_{2}=\left(\underline{Q} A_{1} \oplus \underline{Q} A_{2}, \underline{S} B_{1} \oplus \underline{S} B_{2}\right)$ and $\bar{G}_{1} \oplus \bar{G}_{2}=\left(\bar{Q} A_{1} \oplus \bar{Q} A_{2}, \bar{S} B_{1} \oplus \bar{S} B_{2}\right)$ are neutrosophic graphs, respectively, such that

(i) $\quad \forall\left(v_{1}, v_{2}\right) \in Q A_{1} \times Q A_{2}$

$$
\begin{aligned}
& T_{\left(\overline{\mathrm{Q}} A_{1} \oplus \overline{\mathrm{Q}} A_{2}\right)}\left(v_{1}, v_{2}\right)=\min \left\{T_{\overline{\mathrm{Q}} A_{1}}\left(v_{1}\right), T_{\overline{\mathrm{Q}} A_{2}}\left(v_{2}\right)\right\}, T_{\left(\underline{\mathrm{Q}} A_{1} \oplus \underline{\mathrm{Q}} A_{2}\right)}\left(v_{1}, v_{2}\right)=\min \left\{T_{\underline{Q} A_{1}}\left(v_{1}\right), T_{\underline{Q} A_{2}}\left(v_{2}\right)\right\}, \\
& I_{\left(\overline{\mathrm{Q}} A_{1} \oplus \overline{\mathrm{Q}} A_{2}\right)}\left(v_{1}, v_{2}\right)=\max \left\{I_{\overline{\mathrm{Q}} A_{1}}\left(v_{1}\right), I_{\overline{\mathrm{Q}} A_{2}}\left(v_{2}\right)\right\}, I_{\left(\underline{Q} A_{1} \oplus \underline{Q} A_{2}\right)}\left(v_{1}, v_{2}\right)=\max \left\{I_{\underline{Q} A_{1}}\left(v_{1}\right), I_{\underline{Q} A_{2}}\left(v_{2}\right)\right\}, \\
& F_{\left(\overline{\mathrm{Q}} A_{1} \oplus \overline{\mathrm{Q}} A_{2}\right)}\left(v_{1}, v_{2}\right)=\max \left\{F_{\overline{\mathrm{Q}} A_{1}}\left(v_{1}\right), F_{\overline{\mathrm{Q}} A_{2}}\left(v_{2}\right)\right\}, F_{\left(\underline{Q} A_{1} \oplus \underline{Q} A_{2}\right)}\left(v_{1}, v_{2}\right)=\max \left\{F_{\underline{Q} A_{1}}\left(v_{1}\right), F_{\underline{Q} A_{2}}\left(v_{2}\right)\right\} .
\end{aligned}
$$

(ii) $\forall v_{1} v_{2} \in S B_{2}, v \in Q A_{1}$.

$$
\begin{aligned}
& T_{\left(\bar{S} B_{1} \oplus \bar{S} B_{2}\right)}\left(\left(v, v_{1}\right)\left(v, v_{2}\right)\right)=\min \left\{T_{\bar{Q} A_{1}}(v), T_{\bar{S} B_{2}}\left(v_{1} v_{2}\right)\right\}, \\
& T_{\left(\underline{S} B_{1} \oplus \underline{S} B_{2}\right)}\left(\left(v, v_{1}\right)\left(v, v_{2}\right)\right)=\min \left\{T_{\underline{Q} A_{1}}(v), T_{\underline{S} B_{2}}\left(v_{1} v_{2}\right)\right\}, \\
& I_{\left(\bar{S} B_{1} \oplus \bar{S} B_{2}\right)}\left(\left(v, v_{1}\right)\left(v, v_{2}\right)\right)=\max \left\{I_{\bar{Q} A_{1}}(v), I_{\bar{S} B_{2}}\left(v_{1} v_{2}\right)\right\}, \\
& I_{\left(\underline{S} B_{1} \oplus \underline{S} B_{2}\right)}\left(\left(v, v_{1}\right)\left(v, v_{2}\right)\right)=\max \left\{I_{\underline{Q} A_{1}}(v), I_{\underline{S} B_{2}}\left(v_{1} v_{2}\right)\right\}, \\
& F_{\left(\bar{S} B_{1} \oplus \bar{S} B_{2}\right)}\left(\left(v, v_{1}\right)\left(v, v_{2}\right)\right)=\max \left\{F_{\bar{Q} A_{1}}(v), F_{\bar{S} B_{2}}\left(v_{1} v_{2}\right)\right\}, \\
& F_{\left(\underline{S} B_{1} \oplus \underline{S} B_{2}\right)}\left(\left(v, v_{1}\right)\left(v, v_{2}\right)\right)=\max \left\{F_{\underline{Q} A_{1}}(v), F_{\underline{S} B_{2}}\left(v_{1} v_{2}\right)\right\} .
\end{aligned}
$$


(iii) $\forall v_{1} v_{2} \in S B_{1}, v \in Q A_{2}$.

$$
\begin{aligned}
& T_{\left(\bar{S} B_{1} \oplus \bar{S} B_{2}\right)}\left(\left(v_{1}, v\right)\left(v_{2}, v\right)\right)=\min \left\{T_{\bar{S} B_{1}}\left(v_{1} v_{2}\right), T_{\bar{Q} A_{2}}(v)\right\}, \\
& T_{\left(\underline{S}_{1} \oplus \underline{S} B_{2}\right)}\left(\left(v_{1}, v\right)\left(v_{2}, v\right)\right)=\min \left\{T_{\underline{S}_{1}}\left(v_{1} v_{2}\right), T_{\underline{Q} A_{2}}(v)\right\}, \\
& I_{\left(\bar{S} B_{1} \oplus \bar{S} B_{2}\right)}\left(\left(v_{1}, v\right)\left(v_{2}, v\right)\right)=\max \left\{I_{\bar{S} B_{1}}\left(v_{1} v_{2}\right), I_{\bar{Q} A_{2}}(v)\right\}, \\
& I_{\left(\underline{S} B_{1} \oplus \underline{S} B_{2}\right)}\left(\left(v_{1}, v\right)\left(v_{2}, v\right)\right)=\max \left\{I_{\underline{S}_{1}}\left(v_{1} v_{2}\right), I_{\underline{Q} A_{2}}(v)\right\}, \\
& F_{\left(\bar{S} B_{1} \oplus \bar{S} B_{2}\right)}\left(\left(v_{1}, v\right)\left(v_{2}, v\right)\right)=\max \left\{F_{\bar{S} B_{1}}\left(v_{1} v_{2}\right), F_{\bar{Q} A_{2}}(v)\right\}, \\
& F_{\left(\underline{S} B_{1} \oplus \underline{S} B_{2}\right)}\left(\left(v_{1}, v\right)\left(v_{2}, v\right)\right)=\max \left\{F_{\underline{S} B_{1}}\left(v_{1} v_{2}\right), F_{Q} A_{2}(v)\right\} .
\end{aligned}
$$

(iv) $\forall v_{1} u_{1} \notin S B_{1}, v_{2} u_{2} \in S B_{2}$.

$$
\begin{aligned}
& T_{\left(\bar{S} B_{1} \oplus \bar{S} B_{2}\right)}\left(\left(v_{1}, v_{2}\right)\left(u_{1}, u_{2}\right)\right)=\min \left\{T_{\bar{S} B_{1}}\left(v_{1} u_{1}\right), T_{\bar{Q} A_{2}}\left(v_{2}\right), T_{\bar{Q} A_{2}}\left(u_{2}\right)\right\}, \\
& T_{\left(\underline{S} B_{1} \oplus \underline{S} B_{2}\right)}\left(\left(v_{1}, v_{2}\right)\left(u_{1}, u_{2}\right)\right)=\min \left\{T_{\underline{S} B_{1}}\left(v_{1} u_{1}\right), T_{\underline{Q} A_{2}}\left(v_{2}\right), T_{\underline{Q} A_{2}}\left(u_{2}\right)\right\}, \\
& I_{\left(\bar{S} B_{1} \oplus \bar{S} B_{2}\right)}\left(\left(v_{1}, v_{2}\right)\left(u_{1}, u_{2}\right)\right)=\max \left\{I_{\bar{S}_{B_{1}}}\left(v_{1} u_{1}\right), I_{\bar{Q} A_{2}}\left(v_{2}\right), I_{\bar{Q} A_{2}}\left(u_{2}\right)\right\}, \\
& I_{\left(\underline{S} B_{1} \oplus \underline{S} B_{2}\right)}\left(\left(v_{1}, v_{2}\right)\left(u_{1}, u_{2}\right)\right)=\max \left\{I_{\underline{S} B_{1}}\left(v_{1} u_{1}\right), I_{\underline{Q} A_{2}}\left(v_{2}\right), I_{\underline{Q} A_{2}}\left(u_{2}\right)\right\}, \\
& F_{\left(\bar{S} B_{1} \oplus \bar{S} B_{2}\right)}\left(\left(v_{1}, v_{2}\right)\left(u_{1}, u_{2}\right)\right)=\max \left\{F_{\bar{S} B_{1}}\left(v_{1} u_{1}\right), F_{\bar{Q} A_{2}}\left(v_{2}\right), F_{\bar{Q} A_{2}}\left(u_{2}\right)\right\}, \\
& F_{\left(\underline{S} B_{1} \oplus \underline{S} B_{2}\right)}\left(\left(v_{1}, v_{2}\right)\left(u_{1}, u_{2}\right)\right)=\max \left\{F_{\underline{S} B_{1}}\left(v_{1} u_{1}\right), F_{\underline{Q} A_{2}}\left(v_{2}\right), F_{\underline{Q} A_{2}}\left(u_{2}\right)\right\} .
\end{aligned}
$$

(v) $\forall v_{1} u_{1} \notin S B_{1}, v_{2} u_{2} \in S B_{2}$.

$$
\begin{aligned}
& T_{\left(\bar{S} B_{1} \oplus \bar{S} B_{2}\right)}\left(\left(v_{1}, v_{2}\right)\left(u_{1}, u_{2}\right)\right)=\min \left\{T_{\bar{Q} A_{1}}\left(v_{1}\right), T_{\bar{Q} A_{1}}\left(u_{1}\right), T_{\bar{S} B_{2}}\left(v_{2} u_{2}\right)\right\}, \\
& T_{\left(\underline{S} B_{1} \oplus \underline{S} B_{2}\right)}\left(\left(v_{1}, v_{2}\right)\left(u_{1}, u_{2}\right)\right)=\min \left\{T_{\underline{Q} A_{1}}\left(v_{1}\right), T_{\underline{Q} A_{1}}\left(u_{1}\right), T_{\underline{S} B_{2}}\left(v_{2} u_{2}\right)\right\}, \\
& I_{\left(\bar{S} B_{1} \oplus \bar{S} B_{2}\right)}\left(\left(v_{1}, v_{2}\right)\left(u_{1}, u_{2}\right)\right)=\max \left\{I_{\bar{Q} A_{1}}\left(v_{1}\right), I_{\bar{Q} A_{1}}\left(u_{1}\right), I_{\bar{S} B_{2}}\left(v_{2} u_{2}\right)\right\}, \\
& I_{\left(\underline{S} B_{1} \oplus \underline{S} B_{2}\right)}\left(\left(v_{1}, v_{2}\right)\left(u_{1}, u_{2}\right)\right)=\max \left\{I_{\underline{Q} A_{1}}\left(v_{1}\right), I_{Q} A_{1}\left(u_{1}\right), I_{\underline{S} B_{2}}\left(v_{2} u_{2}\right)\right\}, \\
& F_{\left(\bar{S} B_{1} \oplus \bar{S} B_{2}\right)}\left(\left(v_{1}, v_{2}\right)\left(u_{1}, u_{2}\right)\right)=\max \left\{F_{\bar{Q} A_{1}}\left(v_{1}\right), F_{\bar{Q} A_{1}}\left(u_{1}\right), F_{\bar{S} B_{2}}\left(v_{2} u_{2}\right)\right\}, \\
& F_{\left(\underline{S} B_{1} \oplus \underline{S} B_{2}\right)}\left(\left(v_{1}, v_{2}\right)\left(u_{1}, u_{2}\right)\right)=\max \left\{F_{\underline{Q} A_{1}}\left(v_{1}\right), F_{\underline{Q} A_{1}}\left(u_{1}\right), F_{\underline{S} B_{2}}\left(v_{2} u_{2}\right)\right\} .
\end{aligned}
$$

Example 7. Let $G_{1}=\left(\underline{G}_{1}, \bar{G}_{1}\right)$ and $G_{2}=\left(\underline{G}_{2}, \bar{G}_{2}\right)$ be two neutrosophic soft rough graphs on $V$, where $\underline{G}_{1}=\left(\underline{Q} A_{1}, \underline{S} B_{1}\right)$ and $\bar{G}_{1}=\left(\bar{Q} A_{1}, \bar{S} B_{1}\right)$ are neutrosophic graphs as shown in Figure 6 and $\underline{G}_{2}=\left(\underline{Q} A_{2}, \underline{S} B_{2}\right)$ and $\bar{G}_{2}=\left(\bar{Q} A_{2}, \bar{S} B_{2}\right)$ are neutrosophic graphs as shown in Figure 7 .
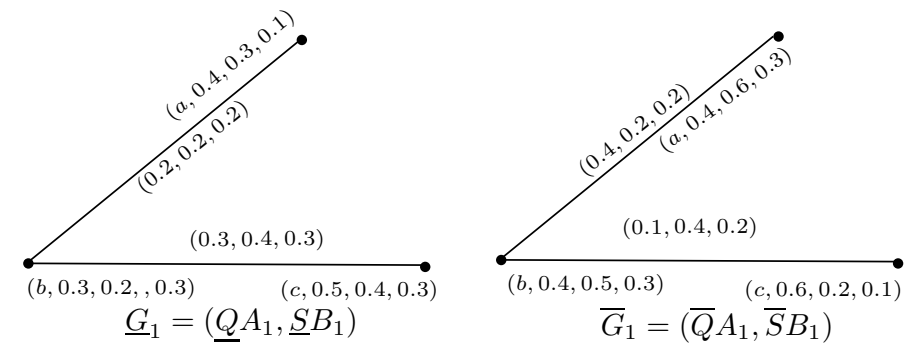

Figure 6. Neutrosophic soft rough graph $G_{1}=\left(\underline{G}_{1}, \bar{G}_{1}\right)$ 

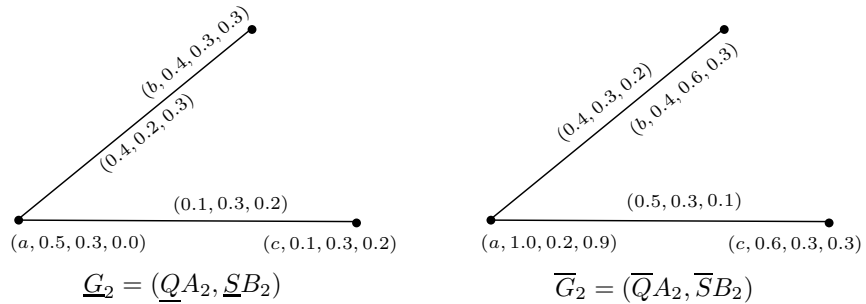

Figure 7. Neutrosophic soft rough graph $G_{2}=\left(\underline{G}_{2}, \bar{G}_{2}\right)$

The symmetric difference of $G_{1}$ and $G_{2}$ is $G=G_{1} \oplus G_{2}=\left(\underline{G}_{1} \oplus \underline{G}_{2}, \bar{G}_{1} \oplus \bar{G}_{2}\right)$, where $\underline{G}_{1} \oplus \underline{G}_{2}=$ $\left(\underline{Q} A_{1} \oplus \underline{Q} A_{2}, \underline{S} B_{1} \oplus \underline{S} B_{2}\right)$ and $\bar{G}_{1} \oplus \bar{G}_{2}=\left(\bar{Q} A_{1} \oplus \bar{Q} A_{2}, \bar{S} B_{1} \oplus \bar{S} B_{2}\right)$ are neutrosophic graphs as shown in Figure 8.
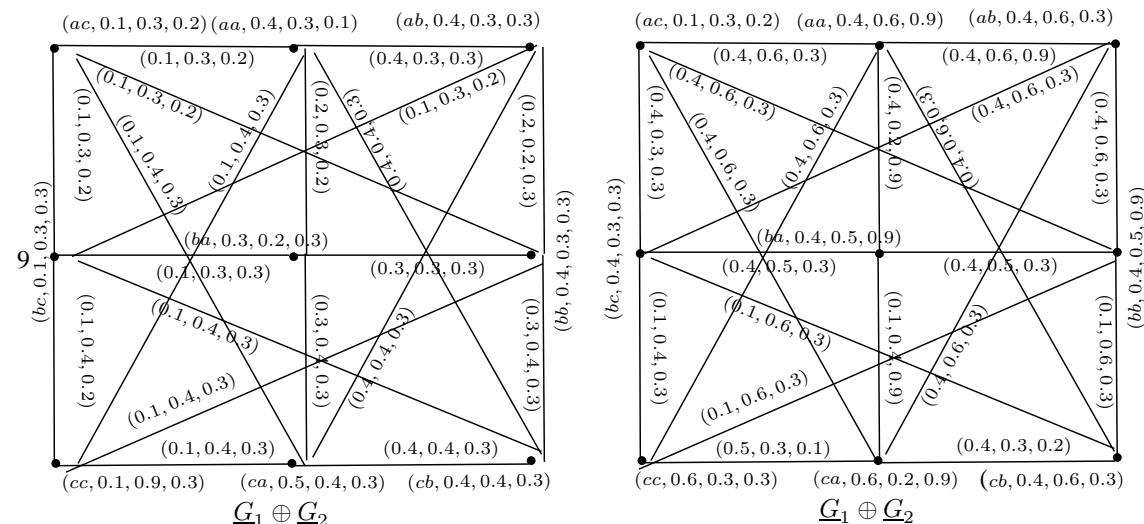

Figure 8. Neutrosophic soft rough graph $G_{1} \oplus G_{2}=\left(\underline{G}_{1} \oplus G_{2}, \bar{G}_{1} \oplus \bar{G}_{2}\right)$

Definition 12. The lexicographic product of $G_{1}$ and $G_{2}$ is a neutrosophic soft rough graph $G=G_{1} \odot G_{2}=\left(G_{1 *} \odot\right.$ $\left.G_{2 *}, G_{1}^{*} \odot G_{2}^{*}\right)$, where $G_{1 *} \odot G_{2 *}=\left(\underline{Q} A_{1} \odot \underline{Q} A_{2}, \underline{S} B_{1} \odot \underline{S} B_{2}\right)$ and $G_{1}^{*} \odot G_{2}^{*}=\left(\bar{Q} A_{1} \odot \bar{Q} A_{2}, \bar{S} B_{1} \odot \bar{S} B_{2}\right)$ are neutrosophic graphs, respectively, such that

(i) $\forall\left(v_{1}, v_{2}\right) \in Q A_{1} \times Q A_{2}$.

$$
\begin{aligned}
& T_{\left(\overline{\mathrm{Q}} A_{1} \odot \overline{\mathrm{Q}} A_{2}\right)}\left(v_{1}, v_{2}\right)=\min \left\{T_{\overline{\mathrm{Q}} A_{1}}\left(v_{1}\right), T_{\overline{\mathrm{Q}} A_{2}}\left(v_{2}\right)\right\}, T_{\left(\underline{Q} A_{1} \odot \underline{Q} A_{2}\right)}\left(v_{1}, v_{2}\right)=\min \left\{T_{\underline{Q} A_{1}}\left(v_{1}\right), T_{\underline{Q} A_{2}}\left(v_{2}\right)\right\}, \\
& I_{\left(\overline{\mathrm{Q}} A_{1} \odot \overline{\mathrm{Q}} A_{2}\right)}\left(v_{1}, v_{2}\right)=\max \left\{I_{\overline{\mathrm{Q}} A_{1}}\left(v_{1}\right), I_{\overline{\mathrm{Q}} A_{2}}\left(v_{2}\right)\right\}, I_{\left(\underline{Q} A_{1} \odot \underline{Q} A_{2}\right)}\left(v_{1}, v_{2}\right)=\max \left\{I_{\underline{Q} A_{1}}\left(v_{1}\right), I_{\underline{Q} A_{2}}\left(v_{2}\right)\right\}, \\
& F_{\left(\overline{\mathrm{Q}} A_{1} \odot \overline{\mathrm{Q}} A_{2}\right)}\left(v_{1}, v_{2}\right)=\max \left\{F_{\overline{\mathrm{Q}} A_{1}}\left(v_{1}\right), F_{\overline{\mathrm{Q}} A_{2}}\left(v_{2}\right)\right\}, F_{\left(\underline{\mathrm{Q}} A_{1} \odot \underline{Q} A_{2}\right)}\left(v_{1}, v_{2}\right)=\max \left\{F_{\underline{Q} A_{1}}\left(v_{1}\right), F_{\underline{Q} A_{2}}\left(v_{2}\right)\right\} .
\end{aligned}
$$

(ii) $\forall v_{1} v_{2} \in S B_{2}, v \in Q A_{1}$.

$$
\begin{aligned}
& T_{\left(\bar{S} B_{1} \odot \bar{S} B_{2}\right)}\left(\left(v, v_{1}\right)\left(v, v_{2}\right)\right)=\min \left\{T_{\bar{Q} A_{1}}(v), T_{\bar{S} B_{2}}\left(v_{1} v_{2}\right)\right\}, \\
& T_{\left(\underline{S} B_{1} \odot \underline{S} B_{2}\right)}\left(\left(v, v_{1}\right)\left(v, v_{2}\right)\right)=\min \left\{T_{\underline{Q} A_{1}}(v), T_{\underline{S} B_{2}}\left(v_{1} v_{2}\right)\right\}, \\
& I_{\left(\bar{S} B_{1} \odot \bar{S} B_{2}\right)}\left(\left(v, v_{1}\right)\left(v, v_{2}\right)\right)=\max \left\{I_{\bar{Q} A_{1}}(v), I_{\bar{S} B_{2}}\left(v_{1} v_{2}\right)\right\}, \\
& I_{\left(\underline{S} B_{1} \odot \underline{S}_{2}\right)}\left(\left(v, v_{1}\right)\left(v, v_{2}\right)\right)=\max \left\{I_{\underline{Q} A_{1}}(v), I_{\underline{S} B_{2}}\left(v_{1} v_{2}\right)\right\}, \\
& F_{\left(\bar{S} B_{1} \odot \bar{S} B_{2}\right)}\left(\left(v, v_{1}\right)\left(v, v_{2}\right)\right)=\max \left\{F_{\bar{Q} A_{1}}(v), F_{\bar{S} B_{2}}\left(v_{1} v_{2}\right)\right\}, \\
& F_{\left(\underline{S} B_{1} \odot \underline{S} B_{2}\right)}\left(\left(v, v_{1}\right)\left(v, v_{2}\right)\right)=\max \left\{F_{\underline{Q} A_{1}}(v), F_{\underline{S} B_{2}}\left(v_{1} v_{2}\right)\right\} .
\end{aligned}
$$


(iii) $\forall v_{1} u_{1} \in S B_{1}, v_{1} u_{2} \in S B_{2}$.

$$
\begin{aligned}
T_{\left(\bar{S} B_{1} \odot \bar{S} B_{2}\right)}\left(\left(v_{1}, v_{1}\right)\left(u_{1}, u_{2}\right)\right) & =\min \left\{T_{\bar{S} B_{1}}\left(v_{1} u_{1}\right), T_{\bar{S} B_{2}}\left(v_{1} u_{2}\right)\right\}, \\
T_{\left(\underline{S} B_{1} \odot \underline{S} B_{2}\right)}\left(\left(v_{1}, v_{1}\right)\left(u_{1}, u_{2}\right)\right) & =\min \left\{T_{\underline{S} B_{1}}\left(v_{1} u_{1}\right), T_{\underline{S} B_{2}}\left(v_{1} u_{2}\right)\right\}, \\
I_{\left(\bar{S} B_{1} \odot \bar{S} B_{2}\right.}\left(\left(v_{1}, v_{1}\right)\left(u_{1}, u_{2}\right)\right) & =\max \left\{I_{\bar{S} B_{1}}\left(v_{1} u_{1}\right), I_{\bar{S} B_{2}}\left(v_{1} u_{2}\right)\right\}, \\
I_{\left(\underline{S} B_{1} \odot \underline{S} B_{2}\right)}\left(\left(v_{1}, v_{1}\right)\left(u_{1}, u_{2}\right)\right) & =\max \left\{I_{\underline{S} B_{1}}\left(v_{1} u_{1}\right), I_{\underline{S}_{2}}\left(v_{1} u_{2}\right)\right\}, \\
F_{\left(\bar{S} B_{1} \odot \bar{S} B_{2}\right.}\left(\left(v_{1}, v_{1}\right)\left(u_{1}, u_{2}\right)\right) & =\max \left\{F_{\bar{S} B_{1}}\left(v_{1} u_{1}\right), F_{\bar{S} B_{2}}\left(v_{1} u_{2}\right)\right\}, \\
F_{\left(\underline{S} B_{1} \odot \underline{S} B_{2}\right)}\left(\left(v_{1}, v_{1}\right)\left(u_{1}, u_{2}\right)\right) & =\max \left\{F_{\underline{S} B_{1}}\left(v_{1} u_{1}\right), F_{\underline{S} B_{2}}\left(v_{1} u_{2}\right)\right\} .
\end{aligned}
$$

Definition 13. The strong product of $G_{1}$ and $G_{2}$ is a neutrosophic soft rough graph $G=G_{1} \otimes G_{2}=\left(G_{1 *} \otimes\right.$ $\left.G_{2 *}, G_{1}^{*} \otimes G_{2}^{*}\right)$, where $G_{1 *} \otimes G_{2 *}=\left(\underline{Q} A_{1} \otimes \underline{Q} A_{2}, \underline{S} B_{1} \otimes \underline{S} B_{2}\right)$ and $G_{1}^{*} \otimes G_{2}^{*}=\left(\bar{Q} A_{1} \otimes \bar{Q} A_{2}, \bar{S} B_{1} \otimes \bar{S} B_{2}\right)$ are neutrosophic graphs, respectively, such that

(i) $\quad \forall\left(v_{1}, v_{2}\right) \in Q A_{1} \times Q A_{2}$.

$$
\begin{aligned}
& T_{\left(\overline{\mathrm{Q}} A_{1} \otimes \overline{\mathrm{Q}} A_{2}\right)}\left(v_{1}, v_{2}\right)=\min \left\{T_{\overline{\mathrm{Q}} A_{1}}\left(v_{1}\right), T_{\overline{\mathrm{Q}} A_{2}}\left(v_{2}\right)\right\}, T_{\left(\underline{\mathrm{Q}} A_{1} \otimes \underline{Q} A_{2}\right)}\left(v_{1}, v_{2}\right)=\min \left\{T_{\underline{\mathrm{Q}} A_{1}}\left(v_{1}\right), T_{\underline{Q} A_{2}}\left(v_{2}\right)\right\}, \\
& I_{\left(\overline{\mathrm{Q}} A_{1} \otimes \overline{\mathrm{Q}} A_{2}\right)}\left(v_{1}, v_{2}\right)=\max \left\{I_{\overline{\mathrm{Q}} A_{1}}\left(v_{1}\right), I_{\overline{\mathrm{Q}} A_{2}}\left(v_{2}\right)\right\}, I_{\left(\underline{Q} A_{1} \otimes \underline{Q} A_{2}\right)}\left(v_{1}, v_{2}\right)=\max \left\{I_{\underline{Q} A_{1}}\left(v_{1}\right), I_{\underline{Q} A_{2}}\left(v_{2}\right)\right\}, \\
& F_{\left(\overline{\mathrm{Q}} A_{1} \otimes \overline{\mathrm{Q}} A_{2}\right)}\left(v_{1}, v_{2}\right)=\max \left\{F_{\overline{\mathrm{Q}} A_{1}}\left(v_{1}\right), F_{\overline{\mathrm{Q}} A_{2}}\left(v_{2}\right)\right\}, F_{\left(\underline{Q} A_{1} \otimes \underline{Q} A_{2}\right)}\left(v_{1}, v_{2}\right)=\max \left\{F_{\underline{Q} A_{1}}\left(v_{1}\right), F_{\underline{Q} A_{2}}\left(v_{2}\right)\right\} .
\end{aligned}
$$

(ii) $\forall v_{1} v_{2} \in S B_{2}, v \in Q A_{1}$.

$$
\begin{aligned}
& T_{\left(\bar{S} B_{1} \otimes \bar{S} B_{2}\right)}\left(\left(v, v_{1}\right)\left(v, v_{2}\right)\right)=\min \left\{T_{\bar{Q} A_{1}}(v), T_{\bar{S} B_{2}}\left(v_{1} v_{2}\right)\right\}, \\
& T_{\left(\underline{S} B_{1} \otimes \underline{S} B_{2}\right)}\left(\left(v, v_{1}\right)\left(v, v_{2}\right)\right)=\min \left\{T_{\underline{Q} A_{1}}(v), T_{\underline{S} B_{2}}\left(v_{1} v_{2}\right)\right\}, \\
& I_{\left(\bar{S} B_{1} \otimes \bar{S} B_{2}\right)}\left(\left(v, v_{1}\right)\left(v, v_{2}\right)\right)=\max \left\{I_{\bar{Q} A_{1}}(v), I_{\bar{S} B_{2}}\left(v_{1} v_{2}\right)\right\}, \\
& I_{\left(\underline{S} B_{1} \otimes \underline{S} B_{2}\right)}\left(\left(v, v_{1}\right)\left(v, v_{2}\right)\right)=\max \left\{I_{\underline{Q} A_{1}}(v), I_{\underline{S}_{2} B_{2}}\left(v_{1} v_{2}\right)\right\}, \\
& F_{\left(\bar{S} B_{1} \otimes \bar{S} B_{2}\right)}\left(\left(v, v_{1}\right)\left(v, v_{2}\right)\right)=\max \left\{F_{\bar{Q} A_{1}}(v), F_{\bar{S} B_{2}}\left(v_{1} v_{2}\right)\right\}, \\
& F_{\left(\underline{S} B_{1} \otimes \underline{S} B_{2}\right)}\left(\left(v, v_{1}\right)\left(v, v_{2}\right)\right)=\max \left\{F_{\underline{Q} A_{1}}(v), F_{\underline{S} B_{2}}\left(v_{1} v_{2}\right)\right\} .
\end{aligned}
$$

(iii) $\forall v_{1} v_{2} \in S B_{1}, v \in Q A_{2}$.

$$
\begin{aligned}
& T_{\left(\bar{S} B_{1} \otimes \bar{S} B_{2}\right)}\left(\left(v_{1}, v\right)\left(v_{2}, v\right)\right)=\min \left\{T_{\bar{S} B_{1}}\left(v_{1} v_{2}\right), T_{\bar{Q} A_{2}}(v)\right\}, \\
& T_{\left(\underline{S}_{1} \otimes \underline{S} B_{2}\right)}\left(\left(v_{1}, v\right)\left(v_{2}, v\right)\right)=\min \left\{T_{\underline{S}_{1}}\left(v_{1} v_{2}\right), T_{\underline{Q} A_{2}}(v)\right\}, \\
& I_{\left(\bar{S} B_{1} \otimes \bar{S} B_{2}\right)}\left(\left(v_{1}, v\right)\left(v_{2}, v\right)\right)=\max \left\{I_{\bar{S} B_{1}}\left(v_{1} v_{2}\right), I_{\bar{Q} A_{2}}(v)\right\}, \\
& I_{\left(\underline{S} B_{1} \otimes \underline{S} B_{2}\right)}\left(\left(v_{1}, v\right)\left(v_{2}, v\right)\right)=\max \left\{I_{\underline{S}_{1}}\left(v_{1} v_{2}\right), I_{\underline{Q} A_{2}}(v)\right\}, \\
& F_{\left(\bar{S} B_{1} \otimes \bar{S} B_{2}\right)}\left(\left(v_{1}, v\right)\left(v_{2}, v\right)\right)=\max \left\{F_{\bar{S} B_{1}}\left(v_{1} v_{2}\right), F_{\bar{Q} A_{2}}(v)\right\}, \\
& F_{\left(\underline{S} B_{1} \otimes \underline{S} B_{2}\right)}\left(\left(v_{1}, v\right)\left(v_{2}, v\right)\right)=\max \left\{F_{\underline{S} B_{1}}\left(v_{1} v_{2}\right), F_{Q} A_{2}(v)\right\} .
\end{aligned}
$$


(iv) $\forall v_{1} u_{1} \in S B_{1}, v_{1} u_{2} \in S B_{2}$.

$$
\begin{aligned}
T_{\left(\bar{S} B_{1} \otimes \bar{S} B_{2}\right)}\left(\left(v_{1}, v_{1}\right)\left(u_{1}, u_{2}\right)\right) & =\min \left\{T_{\bar{S} B_{1}}\left(v_{1} u_{1}\right), T_{\bar{S} B_{2}}\left(v_{1} u_{2}\right)\right\}, \\
T_{\left(\underline{S} B_{1} \otimes \underline{S} B_{2}\right)}\left(\left(v_{1}, v_{1}\right)\left(u_{1}, u_{2}\right)\right) & =\min \left\{T_{\underline{S}_{1}}\left(v_{1} u_{1}\right), T_{\underline{S} B_{2}}\left(v_{1} u_{2}\right)\right\}, \\
I_{\left(\bar{S} B_{1} \otimes \bar{S} B_{2}\right.}\left(\left(v_{1}, v_{1}\right)\left(u_{1}, u_{2}\right)\right) & =\max \left\{I_{\bar{S} B_{1}}\left(v_{1} u_{1}\right), I_{\bar{S} B_{2}}\left(v_{1} u_{2}\right)\right\}, \\
I_{\left(\underline{S} B_{1} \otimes \underline{S} B_{2}\right)}\left(\left(v_{1}, v_{1}\right)\left(u_{1}, u_{2}\right)\right) & =\max \left\{I_{\underline{S} B_{1}}\left(v_{1} u_{1}\right), I_{\underline{S} B_{2}}\left(v_{1} u_{2}\right)\right\}, \\
F_{\left(\bar{S} B_{1} \otimes \bar{S} B_{2}\right.}\left(\left(v_{1}, v_{1}\right)\left(u_{1}, u_{2}\right)\right) & =\max \left\{F_{\bar{S} B_{1}}\left(v_{1} u_{1}\right), F_{\bar{S} B_{2}}\left(v_{1} u_{2}\right)\right\}, \\
F_{\left(\underline{S} B_{1} \otimes \underline{S} B_{2}\right)}\left(\left(v_{1}, v_{1}\right)\left(u_{1}, u_{2}\right)\right) & =\max \left\{F_{\underline{S} B_{1}}\left(v_{1} u_{1}\right), F_{\underline{S} B_{2}}\left(v_{1} u_{2}\right)\right\} .
\end{aligned}
$$

Definition 14. The composition of $G_{1}$ and $G_{2}$ is a neutrosophic soft rough graph $G=G_{1}\left[G_{2}\right]=$ $\left(G_{1 *}\left[G_{2 *}\right], G_{1}^{*}\left[G_{2}^{*}\right]\right)$, where $\left.G_{1 *}\left[G_{2 *}\right]=\left(\underline{Q} A_{1}\left[Q A_{2}\right], \underline{S} B_{1}\left[\underline{S} B_{2}\right]\right)\right]$ and $G_{1}^{*}\left[G_{2}^{*}\right]=\left(\bar{Q} A_{1}\left[\bar{Q} A_{2}\right], \bar{S} B_{1}\left[\bar{S} B_{2}\right]\right)$ are neutrosophic graphs, respectively, such that

(i) $\forall\left(v_{1}, v_{2}\right) \in Q A_{1} \times Q A_{2}$.

$$
\begin{aligned}
& T_{\left(\overline{\mathrm{Q}} A_{1} \times \overline{\mathrm{Q}} A_{2}\right)}\left(v_{1}, v_{2}\right)=\min \left\{T_{\overline{\mathrm{Q}} A_{1}}\left(v_{1}\right), T_{\overline{\mathrm{Q}} A_{2}}\left(v_{2}\right)\right\}, T_{\left(\underline{Q} A_{1} \times \underline{Q} A_{2}\right)}\left(v_{1}, v_{2}\right)=\min \left\{T_{\underline{Q} A_{1}}\left(v_{1}\right), T_{\underline{Q} A_{2}}\left(v_{2}\right)\right\}, \\
& I_{\left(\overline{\mathrm{Q}} A_{1} \times \overline{\mathrm{Q}} A_{2}\right)}\left(v_{1}, v_{2}\right)=\max \left\{I_{\overline{\mathrm{Q}} A_{1}}\left(v_{1}\right), I_{\overline{\mathrm{Q}} A_{2}}\left(v_{2}\right)\right\}, I_{\left(\underline{\mathrm{Q} A} A_{1} \times \underline{Q} A_{2}\right)}\left(v_{1}, v_{2}\right)=\max \left\{I_{\underline{Q} A_{1}}\left(v_{1}\right), I_{\mathrm{Q} A_{2}}\left(v_{2}\right)\right\}, \\
& F_{\left(\overline{\mathrm{Q}} A_{1} \times \overline{\mathrm{Q}} A_{2}\right)}\left(v_{1}, v_{2}\right)=\max \left\{F_{\overline{\mathrm{Q}} A_{1}}\left(v_{1}\right), F_{\overline{\mathrm{Q}} A_{2}}\left(v_{2}\right)\right\}, F_{\left(\underline{Q} A_{1} \times \underline{Q} A_{2}\right)}\left(v_{1}, v_{2}\right)=\max \left\{F_{\underline{Q} A_{1}}\left(v_{1}\right), F_{\underline{Q} A_{2}}\left(v_{2}\right)\right\} .
\end{aligned}
$$

(ii) $\forall v_{1} v_{2} \in S B_{2}, v \in Q A_{1}$.

$$
\begin{aligned}
& T_{\left(\bar{S} B_{1} \times \bar{S} B_{2}\right)}\left(\left(v, v_{1}\right)\left(v, v_{2}\right)\right)=\min \left\{T_{\bar{Q} A_{1}}(v), T_{\bar{S} B_{2}}\left(v_{1} v_{2}\right)\right\}, \\
& T_{\left(\underline{S} B_{1} \times \underline{S} B_{2}\right)}\left(\left(v, v_{1}\right)\left(v, v_{2}\right)\right)=\min \left\{T_{\underline{Q} A_{1}}(v), T_{\underline{S} B_{2}}\left(v_{1} v_{2}\right)\right\}, \\
& I_{\left(\bar{S} B_{1} \times \bar{S} B_{2}\right)}\left(\left(v, v_{1}\right)\left(v, v_{2}\right)\right)=\max \left\{I_{\bar{Q} A_{1}}(v), I_{\bar{S} B_{2}}\left(v_{1} v_{2}\right)\right\}, \\
& I_{\left(\underline{S} B_{1} \times \underline{S} B_{2}\right)}\left(\left(v, v_{1}\right)\left(v, v_{2}\right)\right)=\max \left\{I_{\underline{Q} A_{1}}(v), I_{\underline{S} B_{2}}\left(v_{1} v_{2}\right)\right\}, \\
& F_{\left(\bar{S} B_{1} \times \bar{S} B_{2}\right)}\left(\left(v, v_{1}\right)\left(v, v_{2}\right)\right)=\max \left\{F_{\bar{Q} A_{1}}(v), F_{\bar{S} B_{2}}\left(v_{1} v_{2}\right)\right\}, \\
& F_{\left(\underline{S} B_{1} \times \underline{S} B_{2}\right)}\left(\left(v, v_{1}\right)\left(v, v_{2}\right)\right)=\max \left\{F_{\underline{Q} A_{1}}(v), F_{\underline{S} B_{2}}\left(v_{1} v_{2}\right)\right\} .
\end{aligned}
$$

(iii) $\forall v_{1} v_{2} \in S B_{1}, v \in Q A_{2}$.

$$
\begin{aligned}
& T_{\left(\bar{S} B_{1} \times \bar{S} B_{2}\right)}\left(\left(v_{1}, v\right)\left(v_{2}, v\right)\right)=\min \left\{T_{\bar{S} B_{1}}\left(v_{1} v_{2}\right), T_{\bar{Q} A_{2}}(v)\right\}, \\
& T_{\left(\underline{S} B_{1} \times \underline{S} B_{2}\right)}\left(\left(v_{1}, v\right)\left(v_{2}, v\right)\right)=\min \left\{T_{\underline{S} B_{1}}\left(v_{1} v_{2}\right), T_{\underline{Q} A_{2}}(v)\right\}, \\
& I_{\left(\bar{S} B_{1} \times \bar{S} B_{2}\right)}\left(\left(v_{1}, v\right)\left(v_{2}, v\right)\right)=\max \left\{I_{\bar{S} B_{1}}\left(v_{1} v_{2}\right), I_{\bar{Q} A_{2}}(v)\right\}, \\
& I_{\left(\underline{S} B_{1} \times \underline{S} B_{2}\right)}\left(\left(v_{1}, v\right)\left(v_{2}, v\right)\right)=\max \left\{I_{\underline{S} B_{1}}\left(v_{1} v_{2}\right), I_{\underline{Q} A_{2}}(v)\right\}, \\
& F_{\left(\bar{S} B_{1} \times \bar{S} B_{2}\right)}\left(\left(v_{1}, v\right)\left(v_{2}, v\right)\right)=\max \left\{F_{\bar{S} B_{1}}\left(v_{1} v_{2}\right), F_{\bar{Q} A_{2}}(v)\right\}, \\
& F_{\left(\underline{S} B_{1} \times \underline{S} B_{2}\right)}\left(\left(v_{1}, v\right)\left(v_{2}, v\right)\right)=\max \left\{F_{\underline{S} B_{1}}\left(v_{1} v_{2}\right), F_{Q} A_{2}(v)\right\} .
\end{aligned}
$$


(iv) $\forall v_{1} u_{1} \in S B_{1}, v_{1} \neq u_{2} \in Q A_{2}$.

$$
\begin{aligned}
T_{\left(\bar{S} B_{1} \times \bar{S} B_{2}\right)}\left(\left(v_{1}, v_{1}\right)\left(u_{1}, u_{2}\right)\right) & =\min \left\{T_{\bar{S} B_{1}}\left(v_{1} u_{1}\right), T_{\bar{S} B_{2}}\left(v_{1} u_{2}\right)\right\}, \\
T_{\left(\underline{S} B_{1} \times \underline{S} B_{2}\right)}\left(\left(v_{1}, v_{1}\right)\left(u_{1}, u_{2}\right)\right) & =\min \left\{T_{\underline{S} B_{1}}\left(v_{1} u_{1}\right), T_{\underline{S} B_{2}}\left(v_{1} u_{2}\right)\right\}, \\
I_{\left(\bar{S} B_{1} \times \bar{S} B_{2}\right.}\left(\left(v_{1}, v_{1}\right)\left(u_{1}, u_{2}\right)\right) & =\max \left\{I_{\bar{S} B_{1}}\left(v_{1} u_{1}\right), I_{\bar{S} B_{2}}\left(v_{1} u_{2}\right)\right\}, \\
I_{\left(\underline{S} B_{1} \times \underline{S} B_{2}\right)}\left(\left(v_{1}, v_{1}\right)\left(u_{1}, u_{2}\right)\right) & =\max \left\{I_{\underline{S} B_{1}}\left(v_{1} u_{1}\right), I_{\underline{S} B_{2}}\left(v_{1} u_{2}\right)\right\}, \\
F_{\left(\bar{S} B_{1} \times \bar{S} B_{2}\right.}\left(\left(v_{1}, v_{1}\right)\left(u_{1}, u_{2}\right)\right) & =\max \left\{F_{\bar{S} B_{1}}\left(v_{1} u_{1}\right), F_{\bar{S} B_{2}}\left(v_{1} u_{2}\right)\right\}, \\
F_{\left(\underline{S} B_{1} \times \underline{S} B_{2}\right)}\left(\left(v_{1}, v_{1}\right)\left(u_{1}, u_{2}\right)\right) & =\max \left\{F_{\underline{S} B_{1}}\left(v_{1} u_{1}\right), F_{\underline{S} B_{2}}\left(v_{1} u_{2}\right)\right\} .
\end{aligned}
$$

Definition 15. Let $G=(\underline{G}, \bar{G})$ be a neutrosophic soft rough graph. The complement of $G$, denoted by $\underline{G}=(\underline{G}, \underline{\bar{G}})$ is a neutrosophic soft rough graph, where $\underline{G}=\left(\underline{Q} A, \underline{S^{\prime}} B\right)$ and $\dot{\bar{G}}=(\bar{Q} A, \bar{S} B)$ are neutrosophic graphs such that

(i) $\forall v \in Q A$.

$$
\begin{aligned}
& T_{\overline{\mathrm{Q}} A}^{\prime}(v)=T_{\overline{\mathrm{Q}} A(v)}, \quad I_{\overline{\mathrm{Q}} A}^{\prime}(v)=I_{\overline{\mathrm{Q}} A(v)}, \quad F_{\overline{\mathrm{Q}} A}^{\prime}(v)=F_{\overline{\mathrm{Q}} A(v)}, \\
& T_{\underline{Q} A}^{\prime}(v)=T_{\underline{Q} A(v)}, \quad I_{\underline{Q} A}^{\prime}(v)=I_{\underline{Q} A(v)}, \quad F_{\underline{Q} A}^{\prime}(v)=F_{\underline{Q} A(v)} .
\end{aligned}
$$

(ii) $\forall v, u \in Q A$.

$$
\begin{aligned}
T_{\bar{S} B}^{\prime}(v u) & =\min \left\{T_{\bar{Q} A}(v), T_{\bar{Q} A}(u)\right\}-T_{\bar{S} B}(v u), \\
I_{\bar{S} B}^{\prime}(v u) & =\max \left\{I_{\bar{Q} A}(v), I_{\bar{Q} A}(u)\right\}-I_{\bar{S} B}(v u), \\
F_{\bar{S} B}^{\prime}(v u) & =\max \left\{F_{\bar{Q} A}(v), F_{\bar{Q} A}(u)\right\}-F_{\bar{S} B}(v u), \\
T_{\underline{S} B}^{\prime}(v u) & =\min \left\{T_{\underline{Q} A}(v), T_{\underline{Q} A}(u)\right\}-T_{\underline{S} B}(v u), \\
I_{\underline{S} B}^{\prime}(v u) & =\max \left\{I_{\underline{Q} A}(v), I_{\underline{Q} A}(u)\right\}-I_{\bar{S}_{B}}(v u), \\
F_{\underline{S} B}^{\prime}(v u) & =\max \left\{F_{\underline{Q} A}(v), F_{\underline{Q} A}(u)\right\}-F_{\underline{S}_{B}}(v u) .
\end{aligned}
$$

Example 8. Consider an NSRGs G as shown in Figure 9.
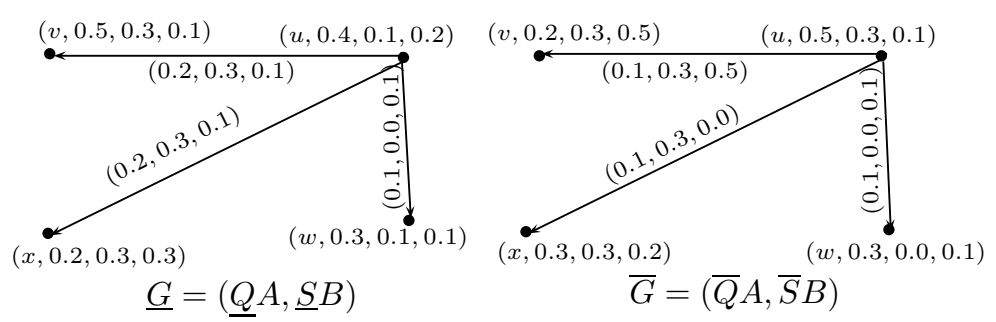

Figure 9. Neutrosophic soft rough graph $G=(\underline{G}, \bar{G})$

The complement of $G$ is $\dot{G}=(\underline{G}, \dot{\bar{G}})$ is obtained by using the Definition 15 , where $\underline{\underline{G}}=(\underline{Q} A, \underline{S} B)$ and $\overline{\bar{G}}=(\bar{Q} A, \bar{S} B)$ are neutrosophic graphs as shown in Figure 10. 

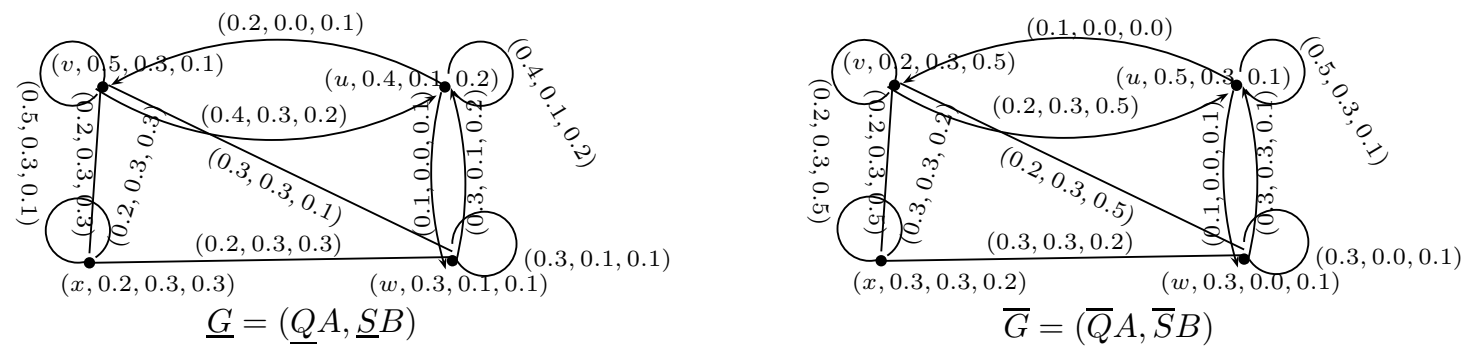

Figure 10. Neutrosophic soft rough graph $\dot{G}=(\underline{G}, \dot{\bar{G}})$

Definition 16. A graph $G$ is called self complement, if $G=\mathbf{G}$, i.e.,

(i) $\forall v \in Q A$.

$$
\begin{aligned}
& T_{\overline{\mathrm{Q}}_{A}}^{\prime}(v)=T_{\overline{\mathrm{Q}} A(v)}, \quad I_{\overline{\mathrm{Q}} A}^{\prime}(v)=I_{\overline{\mathrm{Q}} A(v)}, F_{\overline{\mathrm{Q}} A}^{\prime}(v)=F_{\overline{\mathrm{Q}} A(v)}, \\
& T_{\underline{Q} A}^{\prime}(v)=T_{\underline{Q} A(v)}, I_{\underline{Q} A}^{\prime}(v)=I_{\underline{Q} A(v)}, F_{\underline{Q} A}^{\prime}(v)=F_{\underline{Q} A(v)} .
\end{aligned}
$$

(ii) $\forall v, u \in Q A$.

$$
\begin{aligned}
& T_{\bar{S} B}^{\prime}(v u)=T_{\bar{S} B}(v u), I_{\bar{S} B}^{\prime}(v u)=I_{\bar{S} B}(v u), F_{\bar{S} B}^{\prime}(v u)=F_{\bar{S} B}(v u), \\
& T_{\underline{S} B}^{\prime}(v u)=T_{\underline{S} B}(v u), I_{\underline{S} B}^{\prime}(v u)=I_{\underline{S} B}(v u), F_{\underline{S} B}^{\prime}(v u)=F_{\underline{S} B}(v u) .
\end{aligned}
$$

Definition 17. A neutrosophic soft rough graph $G$ is called strong neutrosophic soft rough graph if $\forall u v \in S B$,

$$
\begin{aligned}
& \left.T_{\bar{S} B}(v u)=\min \left\{T_{\overline{\mathrm{Q}} A}(v), T_{\overline{\mathrm{Q}} A}(u)\right\}, I_{\overline{\mathrm{S}} B}(v u)=\max \left\{I_{\overline{\mathrm{Q}} A}(v), I_{\overline{\mathrm{Q}} A}(u)\right\}\right), F_{\overline{\bar{S}} B}(v u)=\max \left\{F_{\overline{\mathrm{Q}} A}(v), F_{\overline{\mathrm{Q}} A}(u)\right\}, \\
& T_{\underline{S} B}(v u)=\min \left\{T_{\underline{Q} A}(v), T_{\underline{Q} A}(u)\right\}, I_{\underline{S} B}(v u)=\max \left\{I_{\underline{Q} A}(v), I_{\underline{Q} A}(u)\right\}, F_{\underline{S} B}(v u)=\max \left\{F_{\underline{Q} A}(v), F_{\underline{Q} A}(u)\right\} .
\end{aligned}
$$

Example 9. Consider a graph $G$ such that $V=\{u, v, w\}$ and $E=\{u v, v w, w u\}$, as shown in Figure 11. Let $Q A$ be a neutrosophic soft rough set of $V$ and let $S B$ be a neutrosophic soft rough set of E defined in the Tables 9 and 10 , respectively.

Table 9. Neutrosophic soft rough set on $V$.

\begin{tabular}{ccc}
\hline $\boldsymbol{V}$ & $\overline{\mathbf{Q}} \boldsymbol{A}$ & $\underline{\underline{Q} A}$ \\
\hline$u$ & $(0.8,0.5,0.2)$ & $(0.7,0.5,0.2)$ \\
$v$ & $(0.9,0.5,0.1)$ & $(0.7,0.5,0.2)$ \\
$w$ & $(0.7,0.5,0.1)$ & $(0.7,0.5,0.2)$ \\
\hline
\end{tabular}

Table 10. Neutrosophic soft rough set on E.

\begin{tabular}{ccc}
\hline $\boldsymbol{E}$ & $\overline{\boldsymbol{S}} \boldsymbol{B}$ & $\underline{\boldsymbol{S}} \boldsymbol{B}$ \\
\hline$u v$ & $(0.8,0.5,0.2)$ & $(0.7,0.5,0.2)$ \\
$v w$ & $(0.7,0.5,0.1)$ & $(0.7,0.5,0.2)$ \\
$w u$ & $(0.7,0.5,0.2)$ & $(0.7,0.5,0.2)$ \\
\hline
\end{tabular}



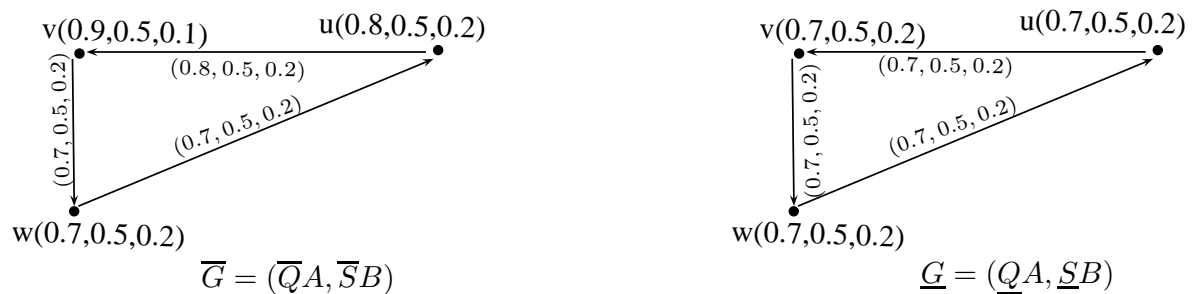

Figure 11. Strong neutrosophic soft rough graph $G=(Q A, S B)$

Hence, $G=(Q A, S B)$ is a strong neutrosophic soft rough graph.

Definition 18. A neutrosophic soft rough graph $G$ is called a complete neutrosophic soft rough graph if $\forall v u \in Q A$,

$$
\begin{aligned}
& T_{\bar{S} B}(v u)=\min \left\{T_{\bar{Q} A}(v), T_{\bar{Q} A}(u)\right\}, I_{\bar{S} B}(v u)=\max \left\{I_{\bar{Q} A}(v), I_{\bar{Q} A}(u)\right\}, F_{\bar{S} B}(v u)=\max \left\{F_{\bar{Q} A}(v), F_{\bar{Q} A}(u)\right\}, \\
& T_{\underline{S} B}(v u)=\min \left\{T_{\underline{Q} A}(v), T_{\underline{Q} A}(u)\right\}, I_{\underline{S} B}(v u)=\max \left\{I_{\underline{Q} A}(v), I_{\underline{Q} A}(u)\right\}, F_{\underline{S} B}(v u)=\max \left\{F_{\underline{Q} A}(v), F_{\underline{Q} A}(u)\right\} .
\end{aligned}
$$

Remark 2. Every complete neutrosophic soft rough graph is a strong neutrosophic soft rough graph. However, the converse is not true.

Definition 19. A neutrosophic soft rough graph $G$ is isolated, if $\forall x, y \in Q A$.

$$
T_{\underline{S} B}(v u)=0, I_{\underline{S} B}(v u)=0, F_{\underline{S} B}(v u)=0, T_{\bar{S} B}(v u)=0, I_{\bar{S} B}(v u)=0, F_{\bar{S} B}(v u)=0 \text {. }
$$

Theorem 1. The rejection of two neutrosophic soft rough graphs is a neutrosophic soft rough graph.

Proof. Let $G_{1}=\left(\underline{G}_{1}, \bar{G}_{1}\right)$ and $G_{2}=\left(\underline{G}_{2}, \bar{G}_{2}\right)$ be two NSRGs. Let $G=G_{1} \mid G_{2}=\left(\underline{G}_{1}\left|\underline{G}_{2}, \bar{G}_{1}\right| \bar{G}_{2}\right)$ be the rejection of $G_{1}$ and $G_{2}$, where $\underline{G}_{1} \mid \underline{G}_{2}=\left(Q A_{1}\left|Q A_{2}, \underline{S} B_{1}\right| \underline{S} B_{2}\right)$ and $\bar{G}_{1} \mid \bar{G}_{2}=\left(\bar{Q} A_{1}\left|\bar{Q} A_{2}, \bar{S} B_{1}\right| \underline{S} B_{2}\right)$. We claim that $G=G_{1} \mid G_{2}$ is a neutrosophic soft rough graph. It is enough to show that $\underline{S} B_{1} \mid \underline{S} B_{2}$ and $\bar{S} B_{1} \mid \bar{S} B_{2}$ are neutrosophic relations on $Q A_{1} \mid Q A_{2}$ and $\bar{Q} A_{1} \mid \bar{Q} A_{2}$, respectively. First, we show that $\underline{S} B_{1} \mid \underline{S} B_{2}$ is a neutrosophic relation on $\underline{Q} A_{1} \mid \underline{Q} A_{2}$.

If $v \in Q A_{1}, v_{1} v_{2} \notin \underline{S} B_{2}$, then

$$
\begin{aligned}
T_{\left(\underline{S} B_{1} \mid \underline{S} B_{2}\right)}\left(\left(v, v_{1}\right)\left(v, v_{2}\right)\right) & =\left(T_{\underline{Q} A_{1}}(v) \wedge\left(T_{\underline{Q} A_{2}}\left(v_{2}\right) \wedge T_{\underline{Q} A_{2}}\left(v_{2}\right)\right)\right) \\
& =\left(T_{\underline{Q} A_{1}}(v) \wedge T_{\underline{Q} A_{2}}\left(v_{2}\right)\right) \wedge\left(T_{\underline{Q} A_{1}}(v) \wedge T_{\underline{Q} A_{2}}\left(v_{2}\right)\right) \\
& =T_{\left(\underline{Q} A_{1} \mid \underline{Q} A_{2}\right)}\left(v, v_{1}\right) \wedge T_{\left(\underline{Q} A_{1} \mid \underline{Q} A_{2}\right)}\left(v, v_{2}\right) \\
T_{\left(\underline{S} B_{1} \mid \underline{S} B_{2}\right)}\left(\left(v, v_{1}\right)\left(v, v_{2}\right)\right) & =T_{\left(\underline{Q} A_{1} \mid \underline{Q} A_{2}\right)}\left(v, v_{1}\right) \wedge T_{\left(\underline{Q} A_{1} \mid \underline{Q} A_{2}\right)}\left(v, v_{2}\right) \\
\text { Similarly, } I_{\left(\underline{S} B_{1} \mid \underline{S} B_{2}\right)}\left(\left(v, v_{1}\right)\left(v, v_{2}\right)\right) & =I_{\left(\underline{Q} A_{1} \mid \underline{Q} A_{2}\right)}\left(v, v_{1}\right) \vee I_{\left(\underline{Q} A_{1} \mid \underline{Q} A_{2}\right)}\left(v, v_{2}\right) \\
F_{\left(\underline{S} B_{1} \mid \underline{S} B_{2}\right)}\left(\left(v, v_{1}\right)\left(v, v_{2}\right)\right) & =F_{\left(\underline{Q} A_{1} \mid \underline{Q} A_{2}\right)}\left(v, v_{1}\right) \vee F_{\left(\underline{Q} A_{1} \mid \underline{Q} A_{2}\right)}\left(v, v_{2}\right) .
\end{aligned}
$$

If $v_{1} v_{2} \notin \underline{S} B_{1}, v \in Q A_{2}$, then

$$
\begin{aligned}
T_{\left(\underline{\underline{S}} B_{1} \mid \underline{S} B_{2}\right)}\left(\left(v_{1}, v\right)\left(v_{2}, v\right)\right) & =\left(\left(T_{\underline{Q} A_{1}}\left(v_{1}\right) \wedge T_{\underline{Q} A_{1}}\left(v_{2}\right)\right) \wedge T_{\underline{Q} A_{2}}(v)\right) \\
& =\left(\left(T_{\underline{Q} A_{1}}\left(v_{1}\right) \wedge T_{\underline{Q} A_{2}}(v)\right) \wedge\left(T_{\underline{Q} A_{1}}\left(v_{2}\right) \wedge T_{\underline{Q} A_{2}}(v)\right)\right) \\
& =T_{\left(\underline{Q} A_{1} \mid \underline{Q} A_{2}\right)}\left(v_{1}, v\right) \wedge T_{\left(\underline{Q} A_{1} \mid \underline{Q} A_{2}\right)}\left(v_{2}, v\right) \\
T_{\left(\underline{S} B_{1} \mid \underline{S} B_{2}\right)}\left(\left(v_{1}, v\right)\left(v_{2}, v\right)\right) & =T_{\left(\underline{Q} A_{1} \mid \underline{Q} A_{2}\right)}\left(v_{1}, v\right) \wedge T_{\left(\underline{Q} A_{1} \mid \underline{Q} A_{2}\right)}\left(v_{2}, v\right) \\
\text { Similarly, } I_{\left(\underline{S} B_{1} \mid \underline{S} B_{2}\right)}\left(\left(v_{1}, v\right)\left(v_{2}, v\right)\right) & =I_{\left(\underline{Q} A_{1} \mid \underline{Q} A_{2}\right)}\left(v_{1}, v\right) \vee I_{\left(\underline{Q} A_{1} \mid \underline{Q} A_{2}\right)}\left(v_{2}, v\right) \\
F_{\left(\underline{S} B_{1} \mid \underline{S} B_{2}\right)}\left(\left(v_{1}, v\right)\left(v_{2}, v\right)\right) & =F_{\left(\underline{Q} A_{1} \mid \underline{Q} A_{2}\right)}\left(v_{1}, v\right) \vee F_{\left(\underline{Q} A_{1} \mid \underline{Q} A_{2}\right)}\left(v_{2}, v\right) .
\end{aligned}
$$


If $v_{1} v_{2} \notin \underline{S} B_{1}, u_{1}, u_{2} \notin \underline{S} B_{2}$, then

$$
\begin{aligned}
& T_{\left(\underline{S} B_{1} \mid \underline{S} B_{2}\right)}\left(\left(v_{1}, u_{1}\right)\left(v_{2}, u_{2}\right)\right)=\left(\left(T_{\underline{Q} A_{1}}\left(v_{1}\right) \wedge T_{\underline{Q} A_{1}}\left(v_{2}\right)\right) \wedge\left(T_{\underline{Q} A_{2}}\left(u_{1}\right) \wedge T_{\underline{Q} A_{2}}\left(u_{2}\right)\right)\right) \\
& =\left(T_{\underline{Q} A_{1}}\left(v_{1}\right) \wedge T_{\underline{Q} A_{2}}\left(u_{1}\right)\right) \wedge\left(T_{\underline{Q} A_{1}}\left(v_{2}\right) \wedge T_{\underline{Q} A_{2}}\left(u_{2}\right)\right) \\
& =T_{\left(Q A_{1} \mid Q A_{2}\right)}\left(v_{1}, u_{1}\right) \wedge T_{\left(Q A_{1} \mid Q A_{2}\right)}\left(v_{2}, u_{2}\right) \\
& T_{\left(\underline{S} B_{1} \mid \underline{S} B_{2}\right)}\left(\left(v_{1}, u_{1}\right)\left(v_{2}, u_{2}\right)\right)=T_{\left(Q A_{1} \mid Q A_{2}\right)}\left(v_{1}, u_{1}\right) \wedge T_{\left(Q A_{1} \mid Q A_{2}\right)}\left(u_{1}, u_{2}\right) \\
& \text { Similarly, } I_{\left(\underline{S} B_{1} \mid \underline{S} B_{2}\right)}\left(\left(v_{1}, u_{1}\right)\left(v_{2}, u_{2}\right)\right)=I_{\left(\underline{Q} A_{1} \mid \underline{Q} A_{2}\right)}\left(v_{1}, u_{1}\right) \vee I_{\left(\underline{Q} A_{1} \mid \underline{Q} A_{2}\right)}\left(u_{1}, u_{2}\right) \\
& F_{\left(\underline{S} B_{1} \mid \underline{S} B_{2}\right)}\left(\left(v_{1}, u_{1}\right)\left(v_{2}, u_{2}\right)\right)=F_{\left(\underline{Q} A_{1} \mid \underline{Q} A_{2}\right)}\left(v_{1}, u_{1}\right) \vee F_{\left(\underline{Q} A_{1} \mid \underline{Q} A_{2}\right)}\left(u_{1}, u_{2}\right) \text {. }
\end{aligned}
$$

Thus, $\underline{S} B_{1} \mid \underline{S} B_{2}$ is a neutrosophic relation on $Q A_{1} \mid Q A_{2}$. Similarly, we can show that $\bar{S} B_{1} \mid \bar{S} B_{2}$ is a neutrosophic relation on $\bar{Q} A_{1} \mid \bar{Q} A_{2}$. Hence, $G$ is a neutrosophic soft rough graph.

Theorem 2. The Cartesian product of two NSRGs is a neutrosophic soft rough graph.

Proof. Let $G_{1}=\left(\underline{G}_{1}, \bar{G}_{1}\right)$ and $G_{2}=\left(\underline{G}_{2}, \bar{G}_{2}\right)$ be two NSRGs. Let $G=G_{1} \ltimes G_{2}=\left(\underline{G}_{1} \ltimes \underline{G}_{2}, \bar{G}_{1} \ltimes \bar{G}_{2}\right)$ be the Cartesian product of $G_{1}$ and $G_{2}$, where $\underline{G}_{1} \ltimes \underline{G}_{2}=\left(\underline{Q} A_{1} \ltimes Q A_{2}, \underline{S} B_{1} \ltimes \underline{S} B_{2}\right)$ and $\bar{G}_{1} \ltimes \bar{G}_{2}=$ $\left(\bar{Q} A_{1} \ltimes \bar{Q} A_{2}, \bar{S} B_{1} \ltimes \underline{S} B_{2}\right)$. We claim that $G=G_{1} \ltimes G_{2}$ is a neutrosophic soft rough graph. It is enough to show that $\underline{S} B_{1} \ltimes \underline{S} B_{2}$ and $\bar{S} B_{1} \ltimes \bar{S} B_{2}$ are neutrosophic relations on $\underline{Q} A_{1} \ltimes Q A_{2}$ and $\bar{Q} A_{1} \ltimes \bar{Q} A_{2}$, respectively. We have to show that $\underline{S} B_{1} \ltimes \underline{S} B_{2}$ is a neutrosophic relation on $Q A_{1} \ltimes Q A_{2}$.

If $v \in Q A_{1}, v_{1} u_{1} \in \underline{S} B_{2}$, then

$$
\begin{aligned}
T_{\left(\underline{S} B_{1} \ltimes \underline{S} B_{2}\right)}\left(\left(v, v_{1}\right)\left(v, u_{1}\right)\right) & =T_{\left(\underline{Q} A_{1}\right)}(v) \wedge T_{\left(\underline{S} B_{2}\right)}\left(v_{1} u_{1}\right) \\
& \leqslant T_{\left(\underline{Q} A_{1}\right)}(v) \wedge\left(T_{\left(\underline{Q} A_{2}\right)}\left(v_{1}\right) \wedge T\left(\underline{Q} A_{2}\right)\left(u_{1}\right)\right) \\
& =\left(T_{\left(\underline{Q} A_{1}\right)}(v) \wedge T_{\left(\underline{Q} A_{2}\right)}\left(v_{1}\right)\right) \wedge\left(T_{\left(\underline{Q} A_{1}\right)}(v) \wedge T_{\left(\underline{Q} A_{2}\right)}\left(u_{1}\right)\right) \\
& =T_{\left(\underline{Q} A_{1} \ltimes \underline{Q} A_{2}\right)}\left(v, v_{1}\right) \wedge T_{\left(\underline{Q} A_{1} \ltimes \underline{Q} A_{2}\right)}\left(v, u_{1}\right) \\
T_{\left(\underline{S} B_{1} \ltimes \underline{S} B_{2}\right)}\left(\left(v, v_{1}\right)\left(v, u_{1}\right)\right) & \leqslant T_{\left(\underline{Q} A_{1} \ltimes \underline{Q} A_{2}\right)}\left(v, v_{1}\right) \wedge T_{\left(\underline{Q} A_{1} \ltimes \underline{Q} A_{2}\right)}\left(v, u_{1}\right) \\
\text { Similarly, } I_{\left(\underline{S} B_{1} \ltimes \underline{S} B_{2}\right)}\left(\left(v, v_{1}\right)\left(v, u_{1}\right)\right) & \leqslant I_{\left(\underline{Q} A_{1} \ltimes \underline{Q} A_{2}\right)}\left(v, v_{1}\right) \vee I_{\left(\underline{Q} A_{1} \ltimes \underline{Q} A_{2}\right)}\left(v, u_{1}\right) \\
F_{\left(\underline{S} B_{1} \ltimes \underline{S} B_{2}\right)}\left(\left(v, v_{1}\right)\left(v, u_{1}\right)\right) & \leqslant F_{\left(\underline{Q} A_{1} \ltimes \underline{Q} A_{2}\right)}\left(v, v_{1}\right) \vee F_{\left(\underline{Q} A_{1} \ltimes \underline{Q} A_{2}\right)}\left(v, u_{1}\right) .
\end{aligned}
$$

If $v_{1} u_{1} \in \underline{S} B_{1}, z \in \underline{Q} A_{2}$, then

$$
\begin{aligned}
T_{\left(\underline{S} B_{1} \ltimes \underline{S} B_{2}\right)}\left(\left(v_{1}, z\right)\left(u_{1}, z\right)\right) & =T_{\left(\underline{S} B_{1}\right)}\left(v_{1} u_{1}\right) \wedge T_{\left(\underline{Q} A_{2}\right)}(z) \\
& \leqslant\left(T_{\left(\underline{Q} A_{1}\right)\left(v_{1}\right) \wedge\left(\underline{Q} A_{1}\right)}\left(u_{1}\right)\right) \wedge T_{\left(\underline{Q} A_{2}\right)}(z) \\
& =T_{\left(\underline{Q} A_{1} \ltimes \underline{Q} A_{2}\right)}\left(v_{1}, z\right) \wedge T_{\left(\underline{Q} A_{1} \ltimes \underline{Q} A_{2}\right)}\left(u_{1}, z\right) \\
T_{\left(\underline{S} B_{1} \ltimes \underline{S} B_{2}\right)}\left(\left(v_{1}, z\right)\left(u_{1}, z\right)\right) & \leqslant T_{\left(\underline{Q} A_{1} \ltimes \underline{Q} A_{2}\right)}\left(v_{1}, z\right) \wedge T_{\left(\underline{Q} A_{1} \ltimes \underline{Q} A_{2}\right)}\left(u_{1}, z\right) \\
\text { Similarly, } I_{\left(\underline{S} B_{1} \ltimes \underline{S} B_{2}\right)}\left(\left(v_{1}, z\right)\left(u_{1}, z\right)\right) & \leqslant I_{\left(\underline{Q} A_{1} \ltimes \underline{Q} A_{2}\right)}\left(v_{1}, z\right) \vee I_{\left(\underline{Q} A_{1} \ltimes \underline{Q} A_{2}\right)}\left(u_{1}, z\right) \\
F_{\left(\underline{S} B_{1} \ltimes \underline{S} B_{2}\right)}\left(\left(v_{1}, z\right)\left(u_{1}, z\right)\right) & \leqslant F_{\left(\underline{Q} A_{1} \ltimes \underline{Q} A_{2}\right)}\left(v_{1}, z\right) \vee F_{\left(\underline{Q} A_{1} \ltimes \underline{Q} A_{2}\right)}\left(u_{1}, z\right) .
\end{aligned}
$$

Therefore, $\underline{S} B_{1} \ltimes \underline{S} B_{2}$ is a neutrosophic relation on $\underline{Q} A_{1} \ltimes Q A_{2}$. Similarly, $\bar{S} B_{1} \ltimes \bar{S} B_{2}$ is a neutrosophic relation on $\bar{Q} A_{1} \ltimes \bar{Q} A_{2}$. Hence, $G$ is a neutrosophic rough graph.

Theorem 3. The cross product of two neutrosophic soft rough graphs is a neutrosophic soft rough graph.

Proof. Let $G_{1}=\left(\underline{G}_{1}, \bar{G}_{1}\right)$ and $G_{2}=\left(\underline{G}_{2}, \bar{G}_{2}\right)$ be two NSRGs. Let $G=G_{1} \odot G_{2}=\left(\underline{G}_{1} \odot \underline{G}_{2}, \bar{G}_{1} \odot \bar{G}_{2}\right)$ be the cross product of $G_{1}$ and $G_{2}$, where $\underline{G}_{1} \odot \underline{G}_{2}=\left(Q A_{1} \odot Q A_{2}, \underline{S} B_{1} \odot \underline{S} B_{2}\right)$ and $\bar{G}_{1} \odot \bar{G}_{2}=\left(\bar{Q} A_{1} \odot\right.$ $\left.\bar{Q} A_{2}, \bar{S} B_{1} \odot \underline{S} B_{2}\right)$. We claim that $G=G_{1} \odot G_{2}$ is a neutrosophic soft rough graph. It is enough to show that $\underline{S} B_{1} \odot \underline{S} B_{2}$ and $\bar{S} B_{1} \odot \bar{S} B_{2}$ are neutrosophic relations on $\underline{Q} A_{1} \odot Q A_{2}$ and $\bar{Q} A_{1} \odot \bar{Q} A_{2}$, respectively. First, we show that $\underline{S} B_{1} \odot \underline{S} B_{2}$ is a neutrosophic relation on $\underline{Q} A_{1} \odot \underline{Q} A_{2}$. 
If $v_{1} u_{1} \in \underline{S} B_{1}, v_{1} u_{2} \in \underline{S} B_{2}$, then

$$
\begin{aligned}
T_{\left(\underline{S} B_{1} \odot \underline{S} B_{2}\right)}\left(\left(v_{1}, v_{1}\right)\left(u_{1}, u_{2}\right)\right) & =T_{\left(\underline{S} B_{1}\right)}\left(v_{1} u_{1}\right) \wedge T_{\left(\underline{S} B_{2}\right)}\left(v_{1} u_{2}\right) \\
& \leqslant\left(T_{\left(\underline{Q} A_{1}\right)}\left(v_{1}\right) \wedge T_{\left(\underline{Q} A_{1}\right)}\left(u_{1}\right) \wedge\left(T_{\left(\underline{Q} A_{2}\right)}\left(v_{1}\right) \wedge T_{\left(\underline{Q} A_{2}\right)}\left(u_{2}\right)\right)\right. \\
& =\left(T_{\left(\underline{Q} A_{1}\right)}\left(v_{1}\right) \wedge T_{\left(\underline{Q} A_{2}\right)}\left(v_{1}\right)\right) \wedge\left(T_{\left(\underline{Q} A_{1}\right)}\left(u_{1}\right) \wedge T_{\left(\underline{Q} A_{2}\right)}\left(u_{2}\right)\right) \\
& =T_{\left(\underline{Q} A_{1} \odot \underline{Q} A_{2}\right)}\left(v_{1}, v_{1}\right) \wedge T_{\left(\underline{Q} A_{1} \odot \underline{Q} A_{2}\right)}\left(u_{1}, u_{2}\right) \\
T_{\left(\underline{S} B_{1} \odot \underline{S} B_{2}\right)}\left(\left(v_{1}, v_{1}\right)\left(u_{1}, u_{2}\right)\right) & \leqslant T_{\left(\underline{Q} A_{1} \odot \underline{Q} A_{2}\right)}\left(v_{1}, v_{1}\right) \wedge T_{\left(\underline{Q} A_{1} \odot \underline{Q} A_{2}\right)}\left(v, u_{2}\right) \\
\text { Similarly, } I_{\left(\underline{S} B_{1} \odot \underline{S} B_{2}\right)}\left(\left(v_{1}, v_{1}\right)\left(u_{1}, u_{2}\right)\right) & \leqslant I_{\left(\underline{Q} A_{1} \odot \underline{Q} A_{2}\right)}\left(v_{1}, v_{1}\right) \vee I_{\left(\underline{Q} A_{1} \odot \underline{Q} A_{2}\right)}\left(v, u_{2}\right) \\
F_{\left(\underline{S} B_{1} \odot \underline{S} B_{2}\right)}\left(\left(v_{1}, v_{1}\right)\left(u_{1}, u_{2}\right)\right) & \leqslant F_{\left(\underline{Q} A_{1} \odot \underline{Q} A_{2}\right)}\left(v_{1}, v_{1}\right) \vee F_{\left(\underline{Q} A_{1} \odot \underline{Q} A_{2}\right)}\left(v, u_{2}\right) .
\end{aligned}
$$

Thus, $\underline{S} B_{1} \odot \underline{S} B_{2}$ is a neutrosophic relation on $\underline{Q} A_{1} \odot Q A_{2}$. Similarly, we can show that $\bar{S} B_{1} \odot \bar{S} B_{2}$ is a neutrosophic relation on $\bar{Q} A_{1} \odot \bar{Q} A_{2}$. Hence, $\bar{G}$ is a neutrosophic soft rough graph.

\section{Application}

In this section, we apply the concept of NSRSs to a decision-making problem. In recent times, the object recognition problem has gained considerable importance. The object recognition problem can be considered as a decision-making problem, in which final identification of objects is founded on a given set of information. A detailed description of the algorithm for the selection of most suitable objects based on an available set of alternatives is given, and purposed decision-making method can be used to calculate lower and upper approximation operators to progress deep concerns of the problem. The presented algorithms can be applied to avoid lengthy calculations when dealing with large number of objects. This method can be applied in various domains for multi-criteria selection of objects.

\section{Selection of Most Suitable Generic Version of Brand Name Medicine}

In the pharmaceutical industry, different pharmaceutical companies develop, produce and discover pharmaceutical medicine (drugs) for use as medication. These pharmaceutical companies deals with "brand name medicine" and "generic medicine". Brand name medicine and generic medicine are bioequivalent, and have a generic medicine rate and element of absorption. Brand name medicine and generic medicine have the same active ingredients, but the inactive ingredients may differ. The most important difference is cost. Generic medicine is less expensive as compared to brand name comparators. Usually, generic drug manufacturers face competition to produce cost less products. The product may possibly be slightly dissimilar in color, shape, or markings. The major difference is cost. We consider a brand name drug " $u=$ Loratadine" used for seasonal allergies medication. Consider

$$
\begin{aligned}
V= & \left\{u_{1}=\text { Triamcinolone, } u_{2}=\text { Cetirizine } /\right. \text { Pseudoephedrine, } \\
& u_{3}=\text { Pseudoephedrine, } u_{4}=\text { loratadine } / \text { pseudoephedrine, } \\
& \left.u_{5}=\text { Fluticasone }\right\}
\end{aligned}
$$

is a set of generic versions of "Loratadine". We want to select the most suitable generic version of Loratadine on the basis of parameters $e_{1}=$ Highly soluble, $e_{2}=$ Highly permeable, $e_{3}=$ Rapidly dissolving. $\mathbb{M}=\left\{e_{1}, e_{2}, e_{3}\right\}$ be a set of paraments. Let $Q$ be a neutrosophic soft relation from $V$ to parameter set $M$, and describe truth-membership, indeterminacy-membership and false-membership degrees of generic version medicine corresponding to the parameters as shown in Table 11. 
Table 11. Neutrosophic soft set $(Q, M)$.

\begin{tabular}{cccccc}
\hline $\boldsymbol{Q}$ & $\boldsymbol{u}_{\mathbf{1}}$ & $\boldsymbol{u}_{\mathbf{2}}$ & $\boldsymbol{u}_{\mathbf{3}}$ & $\boldsymbol{u}_{\mathbf{4}}$ & $\boldsymbol{u}_{\mathbf{5}}$ \\
\hline$e_{1}$ & $(0.4,0.5,0.6)$ & $(0.5,0.3,0.6)$ & $(0.7,0.2,0.3)$ & $(0.5,0.7,0.5)$ & $(0.6,0.5,0.4)$ \\
$e_{2}$ & $(0.7,0.3,0.2)$ & $(0.3,0.4,0.3)$ & $(0.6,0.5,0.4)$ & $(0.8,0.4,0.6)$ & $(0.7,0.8,0.5)$ \\
$e_{3}$ & $(0.6,0.3,0.4)$ & $(0.7,0.2,0.3)$ & $(0.7,0.2,0.4)$ & $(0.8,0.7,0.6)$ & $(0.7,0.3,0.5)$ \\
\hline
\end{tabular}

Suppose $A=\left\{\left(e_{1}, 0.2,0.4,0.5\right),\left(e_{2}, 0.5,0.6,0.4\right),\left(e_{3}, 0.7,0.5,0.4\right)\right\}$ is the most favorable object that is an NS on the parameter set $M$ under consideration. Then, $(\underline{Q}(A), \bar{Q}(A))$ is an NSRS in NSAS $(V, M, Q)$, where

$$
\begin{aligned}
& \bar{Q}(A)=\left\{\left(u_{1}, 0.6,0.5,0.4\right),\left(u_{2}, 0.7,0.4,0.4\right),\left(u_{3}, 0.7,0.4,0.4\right),\left(u_{4}, 0.7,0.6,0.5\right),\left(u_{5}, 0.7,0.5,0.5\right)\right\}, \\
& \underline{Q}(A)=\left\{\left(u_{1}, 0.5,0.6,0.4\right),\left(u_{2}, 0.5,0.6,0.5\right),\left(u_{3}, 0.3,0.3,0.5\right),\left(u_{4}, 0.5,0.6,0.5\right),\left(u_{5}, 0.4,0.5,0.5\right)\right\} . \\
& \quad \text { Let } E=\left\{u_{1} v_{2}, u_{1} u_{3}, u_{4} u_{1}, u_{2} u_{3}, u_{5} u_{3}, u_{2} u_{4}, u_{2} u_{5}\right\} \subseteq \dot{V} \text { and } L=\left\{e_{1} e_{3}, e_{2} e_{1}, e_{3} e_{2}\right\} \subseteq \mathbb{M} . \\
& \text { Then, a neutrosophic soft relation } S \text { on } E(\text { from } L \text { to } E) \text { can be defined as follows in Table 12: }
\end{aligned}
$$

Table 12. Neutrosophic soft relation $S$.

\begin{tabular}{cccccccc}
\hline $\boldsymbol{S}$ & $\boldsymbol{u}_{\mathbf{1}} \boldsymbol{u}_{\mathbf{2}}$ & $\boldsymbol{u}_{\mathbf{1}} \boldsymbol{u}_{\mathbf{3}}$ & $\boldsymbol{u}_{\mathbf{4}} \boldsymbol{u}_{\mathbf{1}}$ & $\boldsymbol{u}_{\mathbf{2}} \boldsymbol{u}_{\mathbf{3}}$ & $\boldsymbol{u}_{\mathbf{5}} \boldsymbol{u}_{\mathbf{3}}$ & $\boldsymbol{u}_{\mathbf{2}} \boldsymbol{u}_{\mathbf{4}}$ & $\boldsymbol{u}_{\mathbf{2}} \boldsymbol{u}_{\mathbf{5}}$ \\
\hline$e_{1} e_{2}$ & $(0.3,0.4,0.2)$ & $(0.4,0.4,0.5)$ & $(0.4,0.4,0.5)$ & $(0.6,0.3,0.4)$ & $(0.4,0.2,0.2)$ & $(0.4,0.4,0.2)$ & $(0.4,0.3,0.4)$ \\
$e_{2} e_{3}$ & $(0.5,0.4,0.1)$ & $(0.4,0.3,0.2)$ & $(0.4,0.3,0.2)$ & $(0.3,0.3,0.2)$ & $(0.6,0.2,0.4)$ & $(0.3,0.2,0.1)$ & $(0.3,0.3,0.2)$ \\
$e_{1} e_{3}$ & $(0.4,0.4,0.1)$ & $(0.4,0.2,0.2)$ & $(0.4,0.2,0.2)$ & $(0.5,0.3,0.3)$ & $(0.4,0.2,0.3)$ & $(0.4,0.3,0.1)$ & $(0.5,0.3,0.2)$ \\
\hline
\end{tabular}

Let $B=\left\{\left(e_{1} e_{2}, 0.2,0.4,0.5\right),\left(e_{2} e_{3}, 0.5,0.4,0.4\right),\left(e_{1} e_{3}, 0.5,0.2,0.5\right)\right\}$ be an NS on $L$ that describes some relationship between the parameters under consideration; then, $S B=(\underline{S} B, \bar{S} B)$ is an NSRR, where

$$
\begin{aligned}
\bar{S} B= & \left\{\left(u_{1} u_{2}, 0.5,0.4,0.4\right),\left(u_{1} u_{3}, 0.4,0.2,0.4\right),\left(u_{4} u_{1}, 0.4,0.2,0.4\right),\left(u_{2} u_{3}, 0.5,0.3,0.4\right),\right. \\
& \left.\left(u_{5} u_{3}, 0.5,0.2,0.4\right),\left(u_{2} u_{4}, 0.4,0.3,0.4\right),\left(u_{2} u_{5}, 0.5,0.3,0.4\right)\right\}, \\
\underline{S} B= & \left\{\left(u_{1} u_{2}, 0.2,0.4,0.4\right)\left(u_{1} u_{3}, 0.5,0.4,0.4\right),\left(u_{4} u_{1}, 0.5,0.4,0.4\right),\left(u_{2} u_{3}, 0.4,0.4,0.5\right),\right. \\
& \left.\left(u_{5} u_{3}, 0.2,0.4,0.4\right),\left(u_{2} u_{4}, 0.2,0.4,0.4\right),\left(u_{2} u_{5}, 0.4,0.4,0.5\right)\right\} .
\end{aligned}
$$

Thus, $G=(\underline{G}, \bar{G})$ is an NSRG as shown in Figure 12 .

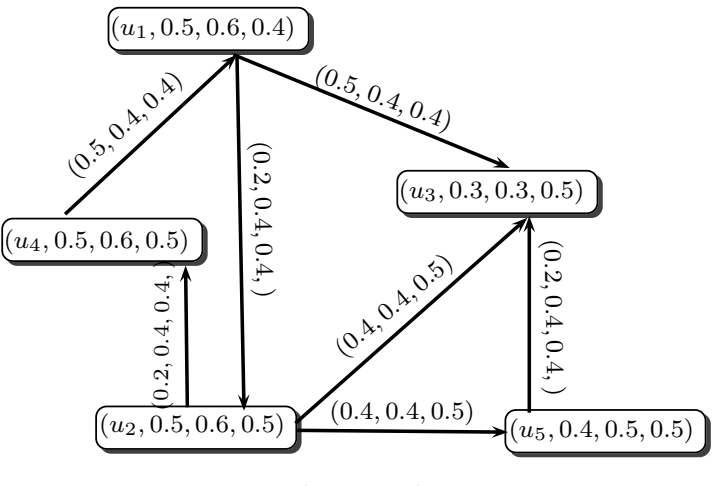

$$
\underline{G}=(\underline{Q} A, \underline{S} B)
$$

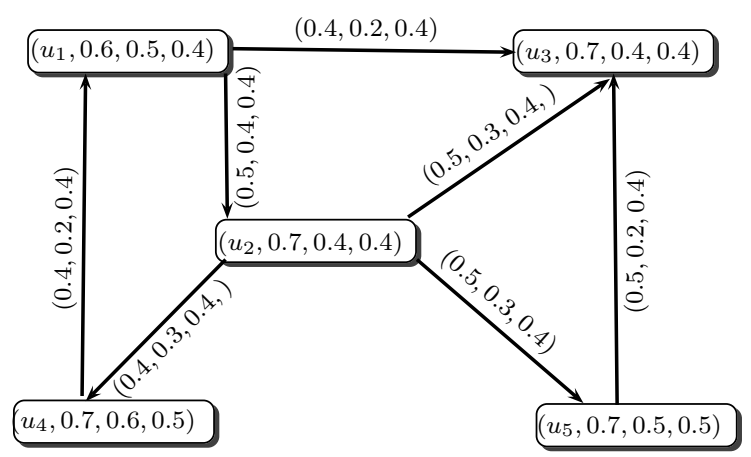

$\bar{G}=(\bar{Q} A, \bar{S} B)$

Figure 12. Neutrosophic soft rough graph $G=(\underline{G}, \bar{G})$

In [3], the sum of two neutrosophic numbers is defined. 
Definition 20. [3] Let $C$ and $D$ be two single valued neutrosophic numbers, and the sum of two single valued neutrosophic number is defined as follows:

$$
C \oplus D=<T_{C}+T_{D}-T_{C} \times T_{D}, I_{C} \times I_{D}, F_{C} \times F_{D}>
$$

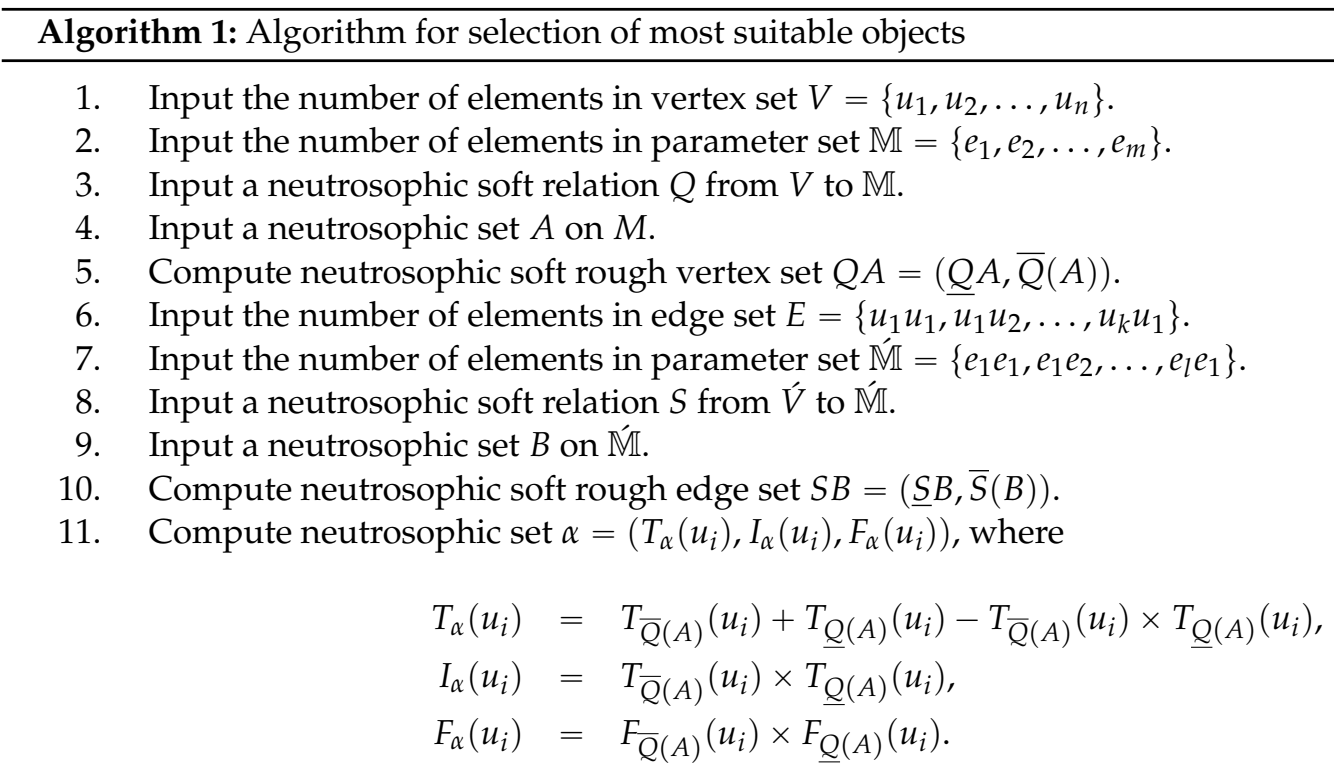

12. Compute neutrosophic set $\beta=\left(T_{\beta}\left(u_{i} u_{i}\right), I_{\beta}\left(u_{i} u_{j}\right), F_{\beta}\left(u_{i} u_{j}\right)\right)$, where

$$
\begin{aligned}
T_{\beta}\left(u_{i} u_{j}\right) & =T_{\bar{S}(B)}\left(u_{i} u_{j}\right)+T_{\underline{S}(B)}\left(u_{i} u_{j}\right)-T_{\bar{S}(B)}\left(u_{i} u_{j}\right) \times T_{\underline{S}(B)}\left(u_{i} u_{j}\right), \\
I_{\beta}\left(u_{i} u_{j}\right) & =T_{\bar{S}(B)}\left(u_{i} u_{j}\right) \times T_{\underline{S}(B)}\left(u_{i} u_{j}\right), \\
F_{\beta}\left(u_{i} u_{j}\right) & =F_{\bar{S}(B)}\left(u_{i} u_{j}\right) \times F_{\underline{S}(B)}\left(u_{i} u_{j}\right) .
\end{aligned}
$$

13. Calculate the score values of each object $u_{i}$, and the score function is defined as follows:

$$
\tilde{S}\left(u_{i}\right)=\sum_{u_{i} u_{j} \in E} \frac{T_{\alpha}\left(u_{j}\right)+I_{\alpha}\left(u_{j}\right)-F_{\alpha}\left(u_{j}\right)}{3-\left(T_{\beta}\left(u_{i} u_{j}\right)+I_{\beta}\left(u_{i} u_{j}\right)-F_{\beta}\left(u_{i} u_{j}\right)\right)} .
$$

14. The decision is $S_{i}$ if $S_{i}=\max _{i=1}^{n} \tilde{S}_{i}$.

15. If $i$ has more than one value, then any one of $S_{i}$ may be chosen.

The sum of UNSRS $\bar{Q} A$ and the LNSRS $\underline{Q} A$ and sum of LNSRR $\underline{S} B$ and the UNSRR $\bar{S} B$ are NSs $\bar{Q} A \oplus \underline{Q} A$ and $\bar{S} B \oplus \underline{S} B$, respectively defined $\overline{b y}$

$$
\begin{aligned}
\alpha=\bar{Q} A \oplus \underline{Q} A= & \left\{\left(u_{1}, 0.8,0.3,0.16\right),\left(u_{2}, 0.85,0.24,0.2\right),\left(u_{3}, 0.79,0.2,0.2\right),\left(u_{4}, 0.85,0.36,0.25\right),\right. \\
& \left.\left(u_{5}, 0.82,0.25,0.25\right)\right\}, \\
\beta=\bar{S} B \oplus \underline{S} B= & \left\{\left(u_{1} u_{2}, 0.6,0.16,0.16\right),\left(u_{1} u_{3}, 0.7,0.8,0.16\right),\left(u_{4} u_{1}, 0.7,0.8,0.16\right),\left(u_{2} u_{3}, 0.7,\right.\right. \\
& \left.0.12,0.2),\left(u_{5} u_{3}, 0.6,0.08,0.16\right),\left(u_{2} u_{4}, 0.52,0.12,0.16\right),\left(u_{2} u_{5}, 0.7,0.12,0.2\right)\right\} .
\end{aligned}
$$

The score function $\tilde{S}\left(u_{k}\right)$ defines for each generic version medicine $u_{i} \in V$,

$$
\tilde{S}\left(u_{i}\right)=\sum_{u_{i} u_{j} \in E} \frac{T_{\alpha}\left(u_{j}\right)+I_{\alpha}\left(u_{j}\right)-F_{\alpha}\left(u_{j}\right)}{3-\left(T_{\beta}\left(u_{i} u_{j}\right)+I_{\beta}\left(u_{i} u_{j}\right)-F_{\beta}\left(u_{i} u_{j}\right)\right)}
$$


and $u_{k}$ with the larger score value $u_{k}=\max _{i} S\left(u_{i}\right)$ is the most suitable generic version medicine. By calculations, we have

$$
\tilde{S}\left(u_{1}\right)=0.88, \tilde{S}\left(u_{2}\right)=0.69, \tilde{S}\left(u_{3}\right)=0.26 \tilde{S}\left(u_{4}\right)=0.57, \text { and } \tilde{S}\left(u_{5}\right)=0.33 \text {. }
$$

Here, $u_{1}$ is the optimal decision, and the most suitable generic version of "Loratadine" is "Triamcinolone". We have used software MATLAB (version 7, MathWorks, Natick, MA, USA) for calculating the required results in the application. The algorithm is given in Algorithm 1. The algorithm of the program is general for any number of objects with respect to certain parameters.

\section{Conclusions}

Rough set theory can be considered as an extension of classical set theory. Rough set theory is a very useful mathematical model to handle vagueness. NS theory, RS theory and SS theory are three useful distinguished approaches to deal with vagueness. NS and RS models are used to handle uncertainty, and combining these two models with another remarkable model of SSs gives more precise results for decision-making problems. In this paper, we have presented the notion of NSRGs and investigated some properties of NSRGs in detail. The notion of NSRGs can be utilized as a mathematical tool to deal with imprecise and unspecified information. In addition, a decision-making method based on NSRGs is proposed. This research work can be extended to (1) Rough bipolar neutrosophic soft sets; (2) Bipolar neutrosophic soft rough sets, (3) Interval-valued bipolar neutrosophic rough sets, and (4) Soft rough neutrosophic graphs.

Author Contributions: Muhammad Akram and Sundas Shahzadi conceived and designed the experiments; Hafsa M. Malik performed the experiments; Florentin Smarandache analyzed the data; Sundas Shahzadi and Hafsa M. Malik wrote the paper.

Conflicts of Interest: The authors declare no conflict of interest.

\section{References}

1. Smarandache, F. Neutrosophic set, a generalisation of the Intuitionistic Fuzzy Sets. Int. J. Pure Appl. Math. 2010, 24, 289-297.

2. Wang, H.; Smarandache, F.; Zhang, Y.; Sunderraman, R. Single-valued neutrosophic sets. Multispace Multistructure 2010, 4, 410-413.

3. Peng, J.J.; Wang, J.Q.; Zhang, H.Y.; Chen, X.H. An outranking approach for multi-criteria decision-making problems with simplified neutrosophic sets. Appl. Soft Comput. 2014, 25, 336-346.

4. Molodtsov, D.A. Soft set theory-first results. Comput. Math. Appl. 1999, 37, 19-31.

5. Maji, P.K.; Biswas, R.; Roy, A.R. Fuzzy soft sets. J. Fuzzy Math. 2001, 9, 589-602.

6. Maji, P.K.; Biswas, R.; Roy, A.R. Intuitionistic fuzzy soft sets. J. Fuzzy Math. 2001, 9, 677-692.

7. Maji, P.K. Neutrosophic soft set. Ann. Fuzzy Math. Inform. 2013, 5, 157-168.

8. Sahin, R.; Kucuk, A. On similarity and entropy of neutrosophic soft sets. J. Intell. Fuzzy Syst. Appl. Eng. Technol. 2014, 27, 2417-2430.

9. Pawlak, Z. Rough sets. Int. J. Comput. Inf. Sci. 1982, 11, 341-356.

10. Feng, F.; Liu, X.; Leoreanu-Fotea, B.; Jun, Y.B. Soft sets and soft rough sets. Inf. Sci. 2011, 181, 1125-1137.

11. Meng, D.; Zhang, X.; Qin, K. Soft rough fuzzy sets and soft fuzzy rough sets. Comput. Math. Appl. 2011, 62, 4635-4645.

12. Sun, B.Z.; Ma, W. Soft fuzzy rough sets and its application in decision making. Artif. Intell. Rev. 2014, 41, $67-80$.

13. Sun, B.Z.; Ma, W.; Liu, Q. An approach to decision making based on intuitionistic fuzzy rough sets over two universes. J. Oper. Res. Soc. 2013, 64, 1079-1089.

14. Zhang, X.; Zhou, B.; Li, Q. A general frame for intuitionistic fuzzy rough sets. Inf. Sci. 2012, 216, 34-49.

15. Zhang, X.; Dai, J.; Yu, Y. On the union and intersection operations of rough sets based on various approximation spaces. Inf. Sci. 2015, 292, 214-229. 
16. Zhang, H.; Shu, L.; Liao, S. Intuitionistic fuzzy soft rough set and its application in decision making. Abstr. Appl. Anal. 2014, 2014, 287314.

17. Zhang, H.; Xiong, L.; Ma, W. Generalized intuitionistic fuzzy soft rough set and its application in decision making. J. Comput. Anal. Appl. 2016, 20, 750-766.

18. Broumi, S.; Smarandache, F. Interval-valued neutrosophic soft rough sets. Int. J. Comput. Math. 2015, 2015, 232919.

19. Broumi, S.; Smarandache, F.; Dhar, M. Rough neutrosophic sets. Neutrosophic Sets Syst. 2014, 3, $62-67$.

20. Yang, H.L.; Zhang, C.L.; Guo, Z.L.; Liu, Y.L.; Liao, X. A hybrid model of single valued neutrosophic sets and rough sets: Single valued neutrosophic rough set model. Soft Comput. 2016, doi:10.1007/s00500-016-2356-y .

21. Akram, M.; Nawaz, S. Operations on soft graphs. Fuzzy Inf. Eng. 2015, 7, 423-449.

22. Akram, M.; Nawaz, S. On fuzzy soft graphs. Ital. J. Pure Appl. Math. 2015, 34, 497-514.

23. Akram, M.; Shahzadi, S. Novel intuitionistic fuzzy soft multiple-attribute decision-making methods. Neural Comput. Appl. 2016, doi:10.1007/s00521-016-2543-x 1-13.

24. Shahzadi, S.; Akram, M. Intuitionistic fuzzy soft graphs with applications. J. Appl. Math. Comput. 2016, 55, 369-392.

25. Akram, M.; Shahzadi, S. Neutrosophic soft graphs with application. J. Intell. Fuzzy Syst. 2017, 2, 841-858.

26. Zafar, F.; Akram, M. A novel decision-making method based on rough fuzzy information. Int. J. Fuzzy Syst. 2017, 1-15, doi:10.1007/s40815-017-0368-0.

27. Peng, T.Z.; Qiang, W.J.; Yu, Z.H. Hybrid single-valued neutrosophic MCGDM with QFD for market segment evaluation and selection. J. Intell. Fuzzy Syst. 2018, 34, 177-187.

28. Qiang, W.J.; Xu, Z.; Yu, Z.H. Hotel recommendation approach based on the online consumer reviews using interval neutrosophic linguistic numbers. J. Intell. Fuzzy Syst. 2018, 34, 381-394.

29. Luo, S.Z.; Cheng, P.F.; Wang, J.Q.; Huang, Y.J. Selecting Project Delivery Systems Based on Simplified Neutrosophic Linguistic Preference Relations. Symmetry 2017, 9, 151, doi:10.3390/sym9070151.

30. Nie, R.X.; Wang, J.Q.; Zhang, H.Y. Solving solar-wind power station location problem using an extended WASPAS technique with Interval neutrosophic sets. Symmetry 2017, 9, 106, doi:10.3390/sym9070106.

31. Wu, X.; Wang, J.; Peng, J.; Qian, J. A novel group decision-making method with probability hesitant interval neutrosphic set and its application in middle level manager's selection. Int. J. Uncertain. Quant. 2018, doi:10.1615/Int.J.UncertaintyQuantification.2017020671.

32. Medina, J.; Ojeda-Aciego, M. Multi-adjoint t-concept lattices. Inf. Sci. 2010, 180, 712-725.

33. Pozna, C.; Minculete, N.; Precup, R.E.; Kóczy, L.T.; Ballagi, Á. Signatures: Definitions, operators and applications to fuzzy modeling. Fuzzy Sets Syst. 2012, 201, 86-104.

34. Nowaková, J.; Prílepok, M.; Snášel, V. Medical image retrieval using vector quantization and fuzzy S-tree. J. Med. Syst. 2017, 41, 1-16.

35. Kumar, A.; Kumar, D.; Jarial, S.K. A hybrid clustering method based on improved artificial bee colony and fuzzy C-Means algorithm. Int. J. Artif. Intell. 2017, 15, 40-60.

(C) 2018 by the authors. Licensee MDPI, Basel, Switzerland. This article is an open access article distributed under the terms and conditions of the Creative Commons Attribution (CC BY) license (http://creativecommons.org/licenses/by/4.0/). 